\title{
WestVirginiaUniversity
}

THE RESEARCH REPOSITORY @ WVU

Graduate Theses, Dissertations, and Problem Reports

2010

\section{The effect of ice crystal shape on aircraft contrails}

Omar E. Meza Castillo

West Virginia University

Follow this and additional works at: https://researchrepository.wvu.edu/etd

\section{Recommended Citation}

Meza Castillo, Omar E., "The effect of ice crystal shape on aircraft contrails" (2010). Graduate Theses, Dissertations, and Problem Reports. 2954.

https://researchrepository.wvu.edu/etd/2954

This Dissertation is protected by copyright and/or related rights. It has been brought to you by the The Research Repository @ WVU with permission from the rights-holder(s). You are free to use this Dissertation in any way that is permitted by the copyright and related rights legislation that applies to your use. For other uses you must obtain permission from the rights-holder(s) directly, unless additional rights are indicated by a Creative Commons license in the record and/ or on the work itself. This Dissertation has been accepted for inclusion in WVU Graduate Theses, Dissertations, and Problem Reports collection by an authorized administrator of The Research Repository @ WVU.

For more information, please contact researchrepository@mail.wvu.edu. 


\title{
THE EFFECT OF ICE CRYSTAL SHAPE ON AIRCRAFT CONTRAILS
}

\author{
Omar E. Meza Castillo \\ Dissertation submitted to the \\ College of Engineering and Mineral Resources \\ at West Virginia University \\ in partial fulfillment of the requirements \\ for the degree of \\ Doctor of Philosophy \\ in \\ Mechanical Engineering
}

Wade W. Huebsch, Ph.D., Co-chair

David C. Lewellen, Ph.D., Co-chair

John M. Kuhlman, Ph.D.

Donald D. Gray, Ph.D.

Gary Morris, Ph.D.

Department of Mechanical and Aerospace Engineering

Morgantown, West Virginia

2010

Keywords: Large-Eddy Simulation, contrails, microphysics, ice crystals, radiation

Copyright, 2010, Omar E. Meza Castillo 


\section{ABSTRACT \\ THE EFFECT OF ICE CRYSTAL SHAPE ON AIRCRAFT CONTRAILS \\ Omar E. Meza Castillo}

Aircraft contrails are a common phenomenon observed in the sky. They are formed mainly of water, from the ambient atmosphere and as a by-product of the combustion process, in the form of ice crystals. They have been identified as a potential contributor to global warming. Some contrails can be long-lived and create man-made cloud cover, thus possibly altering the radiative balance of the earth. There has been a great deal of research on various aspects of contrail development, but to date, little has been done on the influence of ice crystal shapes on the contrail evolution. In-situ studies have reported that young contrails are mainly quasi-spherical crystals while older contrails can have a much more diverse spectrum of possible shapes. The most common shapes found in contrails are quasi-spherical, hexagonal columns, hexagonal plates, and bullet rosettes. Numerical simulations of contrails to date typically have assumed "spherical" as the default ice shape. This work simulated contrail development with a large eddy simulation (LES) model that implemented both spherical and non-spherical shapes to examine the effects. The included shape effect parameters, such as capacitance coefficient, ventilation factor, Kelvin effect, fall velocity and ice crystal surface area, help to establish the shape difference in the results. This study also investigated initial sensitivities to an additional ice parameter, the ice deposition coefficient. The literature shows conflicting values for this coefficient over a wide range.

In the course of this investigation a comparison of various ice metrics was made for simulations with different assumed crystal shapes (spheres, hexagonal columns, hexagonal plates, bullet rosettes and combination of shapes). The simulations were performed at early and late contrail time, with a range of ice crystal sizes, and 
with/without coupled radiation. In young and older contrails and without coupled radiation, the difference from the shape effect in ice crystal number, $\mathrm{N}(\mathrm{t})$, is not significant compared with the level of uncertainty. In young contrails, the difference between spherical and non-spherical shapes in $\mathrm{N}(\mathrm{t})$ is less than $7 \%$ for relatively large ice particles and $23 \%$ for relatively small ice particles. The ice mass, $\mathrm{M}(\mathrm{t})$, is not significantly affected by the crystal shapes, with less than $8 \%$ difference. However, the ice surface area, $\mathrm{S}(\mathrm{t})$, is the ice metric more sensitive to crystal shape, with a maximum difference of $68 \%$. It increases at late time, though it is mainly governed by geometrical rather than dynamical effects.

The small sensitivity to shape effects in the ice contrail metrics when radiation is not included suggests that the spherical shape will provide a reasonable representation for all shapes found in the in-situ studies. The radiation is included at late time, when the lasting effects of contrails are more critical. The inclusion of coupled radiation increases the level of dispersion in the results and hence increases slightly the differences due to shape effects. The small difference is also observed in the infrared heating rates of contrails. 


\section{ACKNOWLEDGEMENTS}

It is a pleasure to acknowledge to Dr. David C. Lewellen and Dr. Wade Huebsch, my advisors who have contributed with their help, support, attention and inspiring scientific discussions which have been very valuable to the completion of this dissertation. They always helped me anytime that I needed guidance in the study of contrail evolution. An special thank to my committee members, Dr. John M. Kuhlman, Dr. Donald D. Gray and Dr. Gary Morris, for their time and valuable comments on the research work and dissertation.

An special thank to my wife Marlene Derteano, and my son Adrian Meza for all this time, their love, support, inspiration and understanding. The absence will not be a constant in the future. At the same time, I would also like to thank my mother Elizabeth and my father Alberto in my homeland, Peru, and my sister-in-law at West Hartford, Connecticut who have been taking care of my wife and son throughout the years. An special thank to my sister Dra. Betty Risco and her mother Delfina Rodriguez. Without their support and understanding, I would not be writing these words today.

I thank to Dr. Eduardo Perez, Dr. Francisco Elizalde-Blancas, Dr. Hermann Alcazar, Jose Antonio Escobar, Aaron Montejo, Orlando Ugarte and students at CFD Lab at West Virginia University for their friendship and help on using some packages. A special thank to Paula L. Beninato at Literacy Volunteers of Great Hartford-Connecticut for the proof-reading of this Dissertation.

This work was carried out at the Department of Mechanical \& Aerospace Engineering at West Virginia University. The project was funded by Boeing to study the contrail evolution below the impact of atmospheric and microphysic parameters. 


\section{Contents}

Abstract $\quad$ ii

Acknowledgements $\quad$ iv

List of Tables viii

List of Figures $\quad$ xi

Nomenclature $\quad$ xii

1 Introduction 1

1.1 Motivation . . . . . . . . . . . . . . . . . 1

1.2 WVU Studies of Contrail Evolution . . . . . . . . . . . . . 4

1.3 Outline of this Study . . . . . . . . . . . . . 6

2 Background of Select Ice Microphysics 9

2.1 Ice Crystal Habits in Contrails . . . . . . . . . . . . . . . . . . . . 9

2.2 Definition of ice crystal shapes . . . . . . . . . . . . . . . 13

2.2.1 Hexagonal Columns: . . . . . . . . . . . . . . . 15

2.2.2 Hexagonal Plates: . . . . . . . . . . . . . . . . . 16

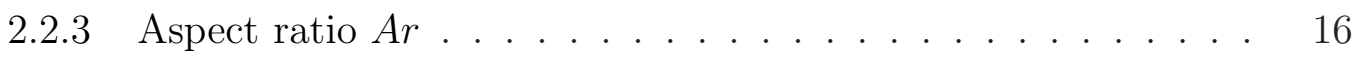

2.2.4 Bullet Rosettes: . . . . . . . . . . . . . . . . 17

2.3 Radiation . . . . . . . . . . . . . . . . . 18 
2.4 Ice Deposition Coefficient $\beta_{i} \ldots \ldots \ldots \ldots$

3 Numerical Model $\quad 21$

3.1 Model Description - Fluid Dynamics _. . . . . . . . . . . . . 21

3.2 Model Description - Ice Microphysics . . . . . . . . . . . . . . 23

3.3 Binned Ice Microphysics . . . . . . . . . . . . . . . . . . 25

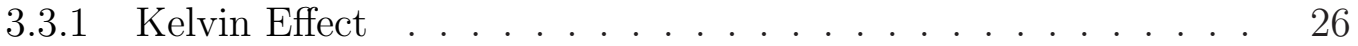

3.3.2 Aspect Ratio and Shape Dimensions _. . . . . . . . . 28

3.3 .3 Ice Surface Area . . . . . . . . . . . . . . . . . . . . . 32

3.3 .4 Combination of shapes: . . . . . . . . . . . . . . . . 32

3.3 .5 Capacitance Factor $C \ldots \ldots \ldots$

3.3.6 Ventilation Coefficient $f_{v} \ldots \ldots \ldots \ldots \ldots \ldots$

3.3 .7 Fall Velocity $V_{\text {fall }} \ldots \ldots \ldots \ldots \ldots \ldots \ldots$

3.3.8 Radiation Effects on Contrails . . . . . . . . . . . . . 43

3.3.9 Ice Deposition Coefficient $\beta_{i} \ldots \ldots \ldots \ldots \ldots$. . . . . 44

4 Contrail Simulation $\quad 47$

4.1 Initial Conditions and Simulation Sequence . . . . . . . . . . 48

4.1 .1 Young Contrails . . . . . . . . . . . . . . . . . . . . . . 49

$4.1 .2 \quad$ Older Contrails . . . . . . . . . . . . . . . . . . . . 49

4.1 .3 Grids . . . . . . . . . . . . . . . . . . . 50

$4.2 \quad$ Ambient Field . . . . . . . . . . . . . . . . . . . . . . . . 51

4.3 Ambient Turbulence and Wind Shear . . . . . . . . . . . . 51

4.4 Description of the Simulation Cases . . . . . . . . . . . . 58

4.5 Three-Dimensional Simulation . . . . . . . . . . . . . . . 61

4.5 .1 Young Contrail Dynamics _. . . . . . . . . . . . . 62

4.5.2 Older Contrail Dynamics . . . . . . . . . . . . . . . . 65 
4.6 Three-Dimensional versus Quasi-2D Simulations . . . . . . . . . 67

5 Shape Effect on Aircraft Contrails $\quad 73$

5.1 Shape Effect Analysis . . . . . . . . . . . . . . . . . 73

5.1.1 Young Contrails . . . . . . . . . . . . . . . 74

5.1 .2 Older Contrails . . . . . . . . . . . . . . . . 78

5.2 Shape Effect Analysis and Initial Radiation Study . . . . . . . . . . . 95

6 Initial Sensitivity Analysis of Ice Deposition Coefficient 106

6.1 Introduction . . . . . . . . . . . . . . 106

6.2 Young Contrails . . . . . . . . . . . . . . . . 107

6.3 Older contrails . . . . . . . . . . . . . . . . 107

7 Conclusions and Future Work 113

7.1 Conclusions . . . . . . . . . . . . . . . . . . . 113

7.2 Future Work . . . . . . . . . . . . . . . . . . . 115 


\section{List of Tables}

2.1 Habits of ice crystals determined by in situ contrail studies . . . . . . 10

3.1 Constants for configurations $4-4$ and $6-2$ to calculate $D_{\max } \ldots \ldots$

3.2 Piecewise constant parameter functions for fall velocity . . . . . . . . 40

4.1 Boeing 767 parameters at the jet exit: . . . . . . . . . . . . 48

4.2 Grids for Contrail Simulations . . . . . . . . . . . . . . 50

4.3 Cases in initial ice shape effect study - Initial Comparison . . . . . . 60

5.1 Maximum shape difference for $T_{\text {ref }}$ and $R H_{\text {ice }}$ BCI - Young contrails . $\quad 77$

5.2 Maximum shape difference for $T_{\text {ref }}$ and $R H_{\text {ice }}$ BCII - Young contrails 78 


\section{List of Figures}

1.1 Natural cirrus and aircraft induced cirrus cloud . . . . . . . . . . 2

2.1 Ice particles size radius and temperature . . . . . . . . . . . . 11

2.2 Ice particle size spectra in contrails . . . . . . . . . . . . . . . . 12

2.3 Ice particle size spectra in young cirrus . . . . . . . . . . . . . . . 13

2.4 Summary of ice habits between $-20^{\circ} \mathrm{C}$ and $-70^{\circ} \mathrm{C} \ldots \ldots . . . . . .14$

2.5 Ice crystal habits . . . . . . . . . . . . . . . 15

2.6 Schematic representation of the hexagonal column and plate shapes . 16

2.7 Schematic representation of the bullet rosette shape . . . . . . . . . 17

3.1 Evolution of ice crystal number distribution with time . . . . . . . 25

3.2 Kelvin effect term for different ice crystal shapes . . . . . . . . . . . . 28

3.3 Aspect ratio of hexagonal column crystals . . . . . . . . . . . . . 29

3.4 Aspect ratio of hexagonal plate crystals . . . . . . . . . . . . . . . 30

3.5 Surface area of single ice crystals . . . . . . . . . . . . . . . 33

3.6 Capacitance of different shapes . . . . . . . . . . . . . . . 35

3.7 Ventilation coefficients of different ice crystal shapes . . . . . . . . . . 37

3.8 Fall velocities for spheres, hexagonal columns, plates, and bullet rosettes 42

3.9 Average logarithm spectral ice bin distribution at late time . . . . . 43

4.1 Simulation Setup - axes and typical grid . . . . . . . . . . . . . 47

4.2 Vertical profiles of $\theta$ used in simulations . . . . . . . . . . 52 
4.3 Vertical profiles of $R H_{i c e}$ used in simulations . . . . . . . . . . . . . 52

4.4 Ambient Turbulence at 1300s early time . . . . . . . . . . . 55

4.5 Ambient Turbulence at 21600s late time . . . . . . . . . . . . . 57

4.6 Simulation, 3D - Young Contrail - Cross-Stream Drift Plot . . . . . . 63

4.7 Total Ice crystal number N(t) and Contrail-mean ice spectrum . . . . 64

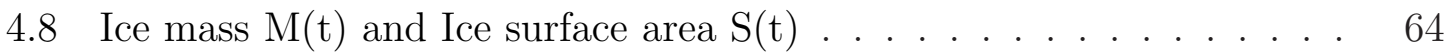

4.9 Simulation, 3D - Young Contrail - Vertical Integrated Drift Plot . . . 65

4.10 Simulation, 3D - Old Contrail - Vertical Integrated Drift Plot . . . . 66

4.11 Simulation, 3D - Old Contrail - Cross-stream Drift Plot . . . . . . . . 67

4.12 3D and Q2D Comparison at early time . . . . . . . . . 69

4.13 3D and Q2D Comparison at late time . . . . . . . . . . . 70

$5.1 \mathrm{~N}(\mathrm{t}), \mathrm{M}(\mathrm{t})$, and $\mathrm{S}(\mathrm{t})$ - case I, shapes, $T_{\text {ref }}$ variation at early time . . 75

$5.2 \mathrm{~N}(\mathrm{t}), \mathrm{M}(\mathrm{t})$, and $\mathrm{S}(\mathrm{t})$ - case I, shapes, $R H_{\text {ice }}$ variation at early time . $\quad 76$

$5.3 \mathrm{~N}(\mathrm{t}), \mathrm{M}(\mathrm{t})$, and $\mathrm{S}(\mathrm{t})$ - case II, shapes, $T_{\text {ref }}$ variation at early time . 79

$5.4 \mathrm{~N}(\mathrm{t}), \mathrm{M}(\mathrm{t})$, and $\mathrm{S}(\mathrm{t})$ - case II, shapes, $R H_{\text {ice }}$ variation at early time . 80

$5.5 \mathrm{~N}(\mathrm{t}), \mathrm{M}(\mathrm{t})$, and $\mathrm{S}(\mathrm{t})$ - case I, shapes, $T_{\text {ref }}$ variation at late time $\ldots . \quad 82$

$5.6 \mathrm{~N}(\mathrm{t}), \mathrm{M}(\mathrm{t})$, and $\mathrm{S}(\mathrm{t})$ - case I, shapes, $R H_{\text {ice }}$ variation at late time . . 83

$5.7 \mathrm{~N}(\mathrm{t}), \mathrm{M}(\mathrm{t})$, and $\mathrm{S}(\mathrm{t})$ - case I, shapes, $R H_{\text {ice }}$ variation at late time . . 84

$5.8 \mathrm{~N}(\mathrm{t}), \mathrm{M}(\mathrm{t})$, and $\mathrm{S}(\mathrm{t})$ - case I, shapes, $R H_{i c e}$ variation at late time . . 85

$5.9 \mathrm{~N}(\mathrm{t}), \mathrm{M}(\mathrm{t})$, and $\mathrm{S}(\mathrm{t})$ - case II, shapes, $T_{\text {ref }}$ variation at late time $\ldots \quad . \quad 87$

$5.10 \mathrm{~N}(\mathrm{t}), \mathrm{M}(\mathrm{t})$, and $\mathrm{S}(\mathrm{t})$ - case II, shapes, $R H_{\text {ice }}$ variation at late time . . 88

$5.11 \mathrm{~N}(\mathrm{t}), \mathrm{M}(\mathrm{t})$ and $\mathrm{S}(\mathrm{t})$, Wind shear - late time - case I . . . . . . . . 90

$5.12 \mathrm{~N}(\mathrm{t}), \mathrm{M}(\mathrm{t})$ and $\mathrm{S}(\mathrm{t})$, Wind shear - late time - case II . . . . . . . 91

$5.13 \mathrm{~N}(\mathrm{t}), \mathrm{M}(\mathrm{t})$ and $\mathrm{S}(\mathrm{t})$, Stratification level - late time - case I . . . . . 93

$5.14 \mathrm{~N}(\mathrm{t}), \mathrm{M}(\mathrm{t})$ and $\mathrm{S}(\mathrm{t})$, Stratification level - late time - case II . . . . . 94

$5.15 \mathrm{~N}(\mathrm{t}), \mathrm{M}(\mathrm{t})$ and $\mathrm{S}(\mathrm{t})$, Radiation - late time - Case I . . . . . . . . 96 
$5.16 \mathrm{~N}(\mathrm{t}), \mathrm{M}(\mathrm{t})$ and $\mathrm{S}(\mathrm{t})$, Radiation - late time - Case II . . . . . . . 97

5.17 Schematic radiation on contrails . . . . . . . . . . . . . . . . 99

5.18 Effective radius . . . . . . . . . . . . . . . . 100

5.19 Volume Absorption Coefficient . . . . . . . . . . . . . . 101

$5.20 \mathrm{~N}(\mathrm{t}), \mathrm{M}(\mathrm{t})$ and $\mathrm{S}(\mathrm{t})$, Infrared heating rates - late time - Case I . . . 103

$5.21 \mathrm{~N}(\mathrm{t}), \mathrm{M}(\mathrm{t})$ and $\mathrm{S}(\mathrm{t})$, Infrared heating rates - late time - Case II . . . 104

6.1 Ice Deposition Coefficient $\beta_{i}$, Large Crystals - Analysis - Early time . 108

6.2 Ice Deposition Coefficient $\beta_{i}$, Small Crystals - Analysis - Early time . 109

6.3 Ice Deposition Coefficient $\beta_{i}$, Large Crystals - Analysis - Late time . . 111

6.4 Ice Deposition Coefficient $\beta_{i}$, Small Crystals - Analysis - Late time . . 112 


\section{Nomenclature}

\section{Roman Symbols}

\begin{tabular}{|c|c|}
\hline$b_{0}, b_{1}, b_{2}, b_{3}$ & coefficients for absorption coefficient \\
\hline$b_{o}$ & vortex-pair separation \\
\hline$b_{t}$ & constant in turbulent dissipation rate \\
\hline$c_{1}, c_{2}, c_{3}$ & parameters for Cunningham correction factor \\
\hline$c_{p}$ & specific heat of air at constant pressure \\
\hline$d$ & twice the side length of the hexagon \\
\hline$e_{s x}$ & saturation vapor pressure with respect to ice \\
\hline$f_{1}, f_{2}$ & correction factors for the Stokes fall velocity \\
\hline$f_{v}$ & ventilation coefficient - Binned model \\
\hline$f_{t}$ & ventilation coefficient for thermal diffusion - Binned model \\
\hline$g$ & acceleration of gravity \\
\hline$g_{0}$ & kernel coefficient without radiation or heat conduction \\
\hline$g_{1}$ & kernel coefficient for heat conduction \\
\hline$h$ & apothem of the hexagonal column \\
\hline$m_{1}, m_{2}, m_{3}$ & transition masses for hexagonal column shape \\
\hline$m_{i}$ & mass of ice particle \\
\hline$m s h_{\text {limit }}$ & mass limit spheres-hexagonal columns \\
\hline$m s h p_{\text {limit }}$ & mass limit spheres-hexagonal plates \\
\hline$m h b_{\text {limit }}$ & mass limit hexagonal columns-bullet rosettes \\
\hline$m_{v}$ & molecular weight of water vapor \\
\hline$n_{b}$ & number of bullets per bullet rosette \\
\hline$q$ & turbulent kinetic energy \\
\hline
\end{tabular}




\section{Roman Symbols}

\begin{tabular}{|c|c|}
\hline$q_{i}$ & ice mixing ratio \\
\hline$q_{s i}$ & ice saturation mixing ratio \\
\hline$q_{t}$ & total water content mixing ratio \\
\hline$q_{v}$ & water vapor mixing ratio \\
\hline$r$ & radius of sphere \\
\hline$r_{e}$ & effective radius \\
\hline$s$ & side of the hexagonal column \\
\hline$t$ & time \\
\hline$w$ & basal face width of the hexagonal column \\
\hline$z_{b}, z_{t}$ & base and top definition in a cloud-free atmosphere \\
\hline$A_{c}$ & ice particle cross-sectional area \\
\hline$A_{c v}$ & ice particle cross-sectional area per unit volume \\
\hline$A_{k}$ & Kelvin curvature effect term \\
\hline$A r$ & aspect ratio of the hexagonal column \\
\hline$C$ & capacitance or crystal shape factor \\
\hline$C_{a}, C_{b}$ & constants for bullet rosette configuration \\
\hline$C_{d}$ & drag coefficient \\
\hline$C f(T, p)$ & correction factor for fall velocity - hexagonal column shape \\
\hline$D$ & water vapor diffusion coefficient \\
\hline$D_{\max }$ & maximum dimension for bullet rosette \\
\hline$D_{c}$ & characteristic max. dimension of ice particle - Reynolds Number \\
\hline$D_{e}$ & effective diameter \\
\hline
\end{tabular}




\section{Roman Symbols}

$D_{g} \quad$ drag force acting on the spherical particle

EI $I_{\text {iceno }}$

emission index of ice crystal number, \#/ $\mathrm{kg}$ of fuel

$F_{b}$

buoyancy force acting on the spherical particle

$F_{b}^{\uparrow}$

upwelling infrared flux at contrail base

$F_{t}^{\downarrow}$

downwelling infrared flux at contrail top

$K_{n}$

particle Knudsen number

$L$

length of hexagonal column ice

$L_{b}$

length of a single ice bullet

$L_{h}$

latent heat of sublimation

$P_{0}$

ref. pressure for hexagonal column fall velocity correction factor

$M(t)$

ice mass per length of flight path

$N(t)$

ice crystal number per length of flight path

$P$

pressure

$P_{o}$

reference pressure

$P_{\text {ref }}$

reference pressure at flight level

$R$

perfect gas constant

Re

particle Reynolds number

$R H_{\text {ice }}$

relative humidity with respect to ice

$S$

supersaturation level - Binned model

$S c$

particle Schmidt number 


\section{Roman Symbols}

$\begin{array}{ll}S_{f} & \text { Cunningham correction for slip-flow } \\ S_{s p h} & \text { ice surface area for a single sphere } \\ S_{b r} & \text { ice surface area for a single bullet rosette } \\ S_{h c / h p} & \text { Ice surface are for a single hexagonal column/plate } \\ S(t) & \text { ice surface area per length of flight path } \\ T & \text { temperature } \\ T_{c} & \text { contrail temperature } \\ T_{0} & \text { ref. pressure for hexagonal column fall velocity correction factor } \\ T_{r e f} & \text { reference temperature at flight level } \\ V & \text { velocity of particle } \\ V_{a} & \text { speed of the aircraft } \\ V_{f a l l} & \text { particle fall velocity - spherical ice shape } \\ V_{o}(m) & \text { hexagonal column particle fall velocity at } T_{0} \text { and } P_{0} \\ V f a l l(m, T, p) & \text { particle fall velocity - hex. column ice shape } \\ V f_{\text {init }} & \text { initial fall velocity of vortex system } \\ W & \text { weight of the spherical particle }\end{array}$




\section{Greek Symbols}

$\alpha$

$\beta$

$\beta_{d}$

$\beta_{a}$

$\beta_{i}$

$\delta$

$\epsilon$

$\epsilon_{i}$

$\epsilon_{t}$

$\eta$

$\Lambda$

$\gamma$

$\kappa^{\uparrow \downarrow}$

$\lambda$

$\mu$

$\rho$

$\rho_{i}$

$\rho_{i e}$

$\sigma$

$\sigma_{i / v}$

$\gamma(m)$

$\delta(m)$

$\Gamma$ constant for the aspect ratio hexagonal column ice shape constant for the aspect ratio hexagonal column ice shape diffusivity factor

volume absorption coefficient

ice deposition coefficient

characteristic length of ice shape

ratio between molar masses of dry air and water vapor

emissivity

turbulent dissipation rate

overall propulsion efficiency of the aircraft

subgrid turbulence length scale

spectral coefficient for mass absorption coefficient

effective mass absorption coefficient

thermal conductivity

dynamic viscosity of air

density of air

density of ice

equilibrium ice density at flight level

Stefan-Boltzman constant

surface tension at ice/vapor interface

piecewise constant function for fall velocity - hex. column shape

piecewise constant function for fall velocity - hex. column shape

vortex circulation 


$\begin{array}{ll}\text { Abbreviations } & \\ \text { IWC } & \text { Ice water content } \\ \text { IWP } & \text { Ice water path } \\ \text { IHR } & \text { Infrared heating rate } \\ \text { IPCC } & \text { Intergovernmental Panel on Climate Change } \\ \text { LW } & \text { Longwave radiation } \\ \text { NASA } & \text { National Aeronautics and Space Administration } \\ \text { SW } & \text { Shortwave radiation } \\ \text { TRADEOFF } & \text { Aircraft emissions: Contribution of different climate } \\ & \text { components to changes in radiative forcing - } \\ & \text { Tradeoff to reduce atmospheric impact } \\ & \text { West Virginia University }\end{array}$




\section{Chapter 1}

\section{Introduction}

\subsection{Motivation}

Contrails are artificial clouds that consist of ice crystals formed behind a passing aircraft. Depending on suitable atmospheric conditions, the morphology of contrails observed in the sky could be widely varied from simple linear dissipating contrails, to spreading persistent contrails (contrail-cirrus). The formation of contrails in the atmosphere has increased at the same rate as the air traffic in the past two decades. Analysis of the future demand indicates for the next 20 years (period 2008-2027), that passenger and cargo traffic will grow at 5.0 and $5.8 \%$ per year respectively (Boeing, 2008). Studies indicate that the projected large increases in air traffic in the coming decades will impact the global occurrence of aircraft contrails. The increase in contrails could significantly alter the actual cloud cover, leading to changes in climate (Penner et al., 1999). The important contribution of these man made clouds to global warming in recent years, has renewed the interest of many investigators regarding the formation, evolution, lasting effects, and global impact of contrails in the atmosphere.

During their evolution into cirrus clouds, the microphysical and radiative properties of contrails can vary significantly; not only with environmental conditions but 
also with time (Schumann and Wendling, 1990). Aged contrails can have similar morphology to natural cirrus as is shown in figure 1.1. In situ studies found that ice crystals evolve in size in young contrails (age $<1 \mathrm{~min}$ ) during their evolution into aged contrails (up to 1 hour), and aged contrails into young cirrus clouds (Schröder et al., 2000).
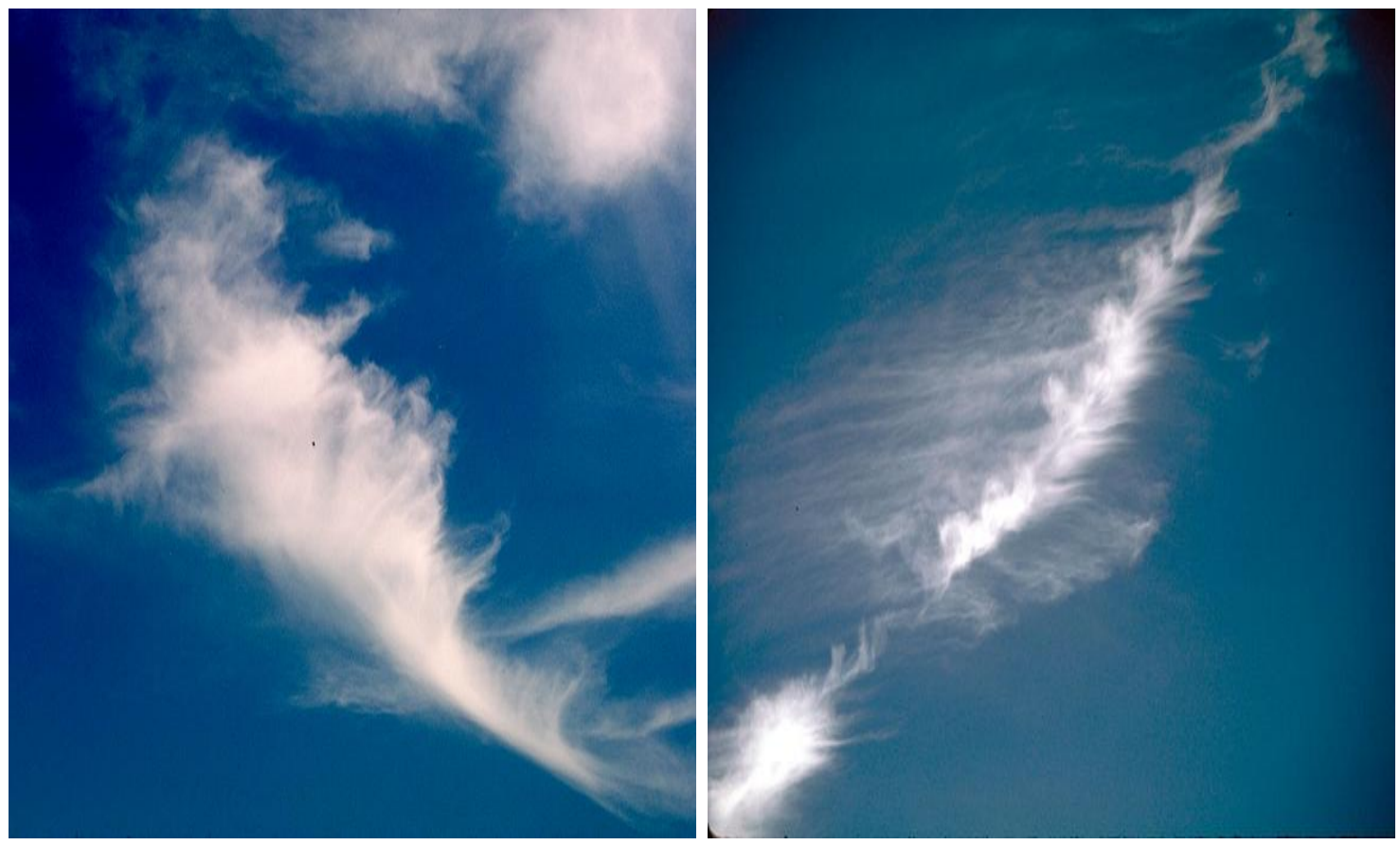

Figure 1.1: At the left a natural cirrus and on the right a cirrus cloud from spreading contrail, image credit: provided by www.maltaweather.info/cirrus.jpg and Cloudman's Cloud Gallery www.cloudman.com/.

Many scientists have suspected that aircraft contrails are implicated in global warming due to the continuous air traffic growth. The non-fly period (from September 11 to September 14) after the terrorist attacks of September 11, 2001, was an opportune event to test in real manner the idea of contrail impact on the climate. Researchers involved in contrail studies had the opportunity to analyze the changes in Earth's surface temperature. The results showed that contrails can be cooling or warming clouds. This means that they can suppress both daytime highs (by reflecting 
sunlight back to space) and nighttime lows (by trapping radiated heat). The final balance suggests that contrails have a net warming effect (Travis et al., 2004).

In the past two decades, there have been modeling studies on contrail evolution that have contributed to our understanding of aircraft contrail induced clouds (e.g. Gierens (1996); Gierens and Jensen (1998); Chlond (1998); Jensen et al. (1998); Sussmann and Gierens (1999); Chen and Lin (2001); Lewellen and Lewellen (2001a); Huebsch and Lewellen (2006b); Unterstrasser et al. (2008)). Models of diverse complexity have been used; some with thermodynamical, dynamical, microphysical and radiative effects. It is not completely understood what are the critical parameters (atmospheric ambient condition, aircraft or microphysical) that affect the global impact.

In this thesis, we are specifically interested in the effects of ice crystal habit (shape and size) on the evolution of contrails from a few seconds out to several hours.

The shapes of ice crystals have been a subject of great interest for a long time. In order to simplify the shape of ice particles, most previous contrail simulations approximated the ice crystal as spherical. But situ measurement have shown that the morphology of ice crystals inside of persistent contrails is complex; the core and periphery have different concentration and ice crystal habits (unresolved spherical, irregular column and bullet rosette) (Lawson et al., 1998). If we want to model in the most realistic manner the formation and evolution of persistent contrails, the simulation of contrails needs to include accurate microphysical properties (such as ice crystal habit and number density) involved in the real phenomenon.

During this research the following questions will be addressed: How do ice microphysical properties affect contrail evolution? Are there specific microphysical properties associated with the lasting effects and formation of persistent contrails? Will the ice crystal shape significantly alter the formation and evolution of persistent con- 
trails into cirrus clouds? Will the ice crystal shape have a significant influence in the simulation at early and late time?

The study of ice crystal shape effects in contrails is potentially important for several reasons: 1) A non-spherical shape involves features that at late time and under specific atmospheric conditions could have a significant influence in the contrail evolution; 2) the optical depth and the radiative effects of contrails depend on size, number and shape of ice crystals; 3) differences between spherical and non-spherical habits could help to understand contrail evolution at early and late time in a realistic manner.

In the process to define a relevant parameter involved in the contrail evolution, the ice crystal shape was selected as a priority. However, in the spectrum of microphysical parameters involved in the evolution of cirrus clouds, the deposition coefficient was detected as a critical parameter (Lin et al., 2002). Lasting effects of cirrus clouds in the upper troposphere have been studied using a wide range of values for the deposition coefficient. This parameter is defined as the probability for an individual water vapor molecule impacting on the crystal to be incorporated into the crystal (Pruppacher and Klett, 1997). The high level of uncertainty in the results for this parameter give us the possibility to study in a initial stage its influence on the contrail evolution. As contrails are mainly formed by ice crystals in transition to contrailscirrus, the deposition coefficient may affect the sensitivity in the ice crystal number remaining in the plume and hence its ice mass, ice surface area and lifetime.

\subsection{WVU Studies of Contrail Evolution}

This thesis work builds upon a longstanding and extensive research effort at WVU originally funded by NASA, and currently under Boeing support. In this part of the dissertation, it is briefly summarize some of this work, much of which is as yet 
unpublished.

The starting point is placed in the studies of the effects of aircraft wake dynamics on species dispersion, wake plume chemistry and contrail development performed by Lewellen and Lewellen (1996); Lewellen et al. (1998); Lewellen and Lewellen (2001a,b), with funding from NASA as part of the Atmospheric Effects of Aviation Project (AEAP). Later work included substantial improvement in the WVU contrail code, extensive sensitivity studies of young and old contrails to atmospheric and aircraft variables, studies of precipitation, coupled radiation, and ice crystal loss.

The WVU contrail code is a three-dimensional high-resolution large-eddy simulation (LES) code that was developed to investigate both the properties of contrails in the near-field plume and far-field evolution out to ages of many hours (Lewellen and Lewellen, 2001a).

The current code initializes simulations using flow and emission fields from Boeing 2D wake simulations. A binned ice microphysics using parts of the NASA Ames Community Aerosol and Radiation Model for Atmospheres (CARMA) is used for ice growth, sedimentation and coupled radiation.

A major modification in the code was the inclusion of "dynamic ice binning" that allows one to define the ice bin structure locally for each point in space and time when the CARMA microphysics is used. In turn, the simulation only needs a subset of the total bins at a given grid location to resolve the ice spectrum, thus reducing overall computational resources (Lewellen et al., 2009).

The most important capabilities of the WVU Contrail code for simulation of aircraft contrails include:

- input of cross-stream and downstream wind shear

- use of binned or bulk ice microphysics

- addition of radiation 
- handle long-term simulations including ice precipitation

- option for jet asymmetry

- use of ambient data file to specify vertical profiles of potential temperature, $R H_{\text {ice }}$ (or total water mixing ratio), and wind shear

- use of passive scalars independent or in addition to the ice

- a scheme was developed to maintain ambient atmospheric turbulence at desired levels at late times.

- extensive options for output diagnostics and post-simulation analysis of results

Recent results are documented in an unpublished collection of reports presented to Boeing, that include analysis of contrails at early and late time, but using only the spherical ice crystal shape. The information available about the ice habits establishes that inside of contrails the most common ice crystal shapes are spheres, hexagonal columns, hexagonal plates, and bullet rosettes. A more realistic treatment should be an initial spectrum formed by a combination of shapes to cover all ice habits found in real contrails, or at minimum allow different ice shapes at later stages of the evolution.

\subsection{Outline of this Study}

The primary focus of this sensitivity study is to improve the fundamental understanding of ice crystal shape effects on the evolution of persistent contrails by extending the present WVU contrail code to simulate contrail evolution using different ice crystal shapes. The non-spherical shape will simulate in a realistic manner most of the important microphysical process involved in the particular habit. The specific goals for this work are to: 
1. Implement realistic modeling of new ice crystal shapes in the WVU contrail code: hexagonal column, hexagonal plate, bullet rosette, and an attempt to create a realistic combined ice spectrum formed by hexagonal column and bullet rosette shapes.

2. Develop an early and late time uncertainty and sensitivity study of some parameters, such as temperature, relative humidity with respect to ice, wind shear, and ambient temperature stratification for every shape in the study.

3. Perform a comparative study of contrail evolution for a Boeing-767 aircraft. The study will have as a point of reference the results with spherical shape. This comparative study will consist of two stages:

- First, two cases will be proposed for study (contrail with small and large ice crystals) to see the effects in the contrail evolution at early time (from 0 to $1000 \mathrm{~s}$ ), where the aircraft wake dynamics are dominated by the pair of downward traveling vortices.

- Second, at late time the previous segment of simulation will be continued in a larger domain to study the evolution of older contrails for several hours.

4. Analyze and determine the differences obtained in the comparative study and establish the effects of crystal shape in the contrail evolution at early and late time.

5. Analyze microphysical processes such as vapor-diffusional growth or sublimation to understand the influence of the shape effect in contrail ice evolution.

6. Attempt the implementation of coupled radiation into the WVU contrail code for the new shapes and analyze the impact of radiation on contrails formed by 
non-spherical ice crystals.

7. Study the influence of deposition coefficient in the contrail evolution through simulation tests.

Chapter 2 of this dissertation presents some necessary background on ice microphysics, the implementation of hexagonal column, hexagonal plate, bullet rosette and combination of shapes in the WVU contrail code is in chapter 3, contrail simulation is explained in chapter 4, the shape effect on contrails is presented in chapter 5, in chapter 6 an initial sensitivity study on ice deposition coefficient is discussed, and conclusions and proposed future work are presented in chapter 7. 


\section{Chapter 2}

\section{Background of Select Ice}

\section{Microphysics}

\subsection{Ice Crystal Habits in Contrails}

Accurate models for the microphysical properties of contrails are important in the simulation of contrail evolution. In the last three decades field observations have shown a wide variety of ice crystals habits in contrails and contrails in transition to cirrus clouds, but still many uncertainties exist. These are mainly in the size measurements of ice crystals, where the approximation to spheres introduces uncertainties in the order of $20 \%$ to $60 \%$ (Baumgardner et al., 1992; Spinhirne et al., 1998); and the unresolved combination of ice crystal shapes, sizes and orientation permit uncertainties in the radiative properties of contrail cirrus (Liou et al., 1998).

Ice crystal habit in contrail and natural cirrus is primarily controlled by humidity and temperature evolution inside the parcel. Previous contrail studies are presented in table 2.1 that lists the shapes, size range, number density, and age found in the in situ microphysical research. The simpler habit consists of particles with spherical or quasispherical shape, while the complex are bullet rosettes, columns, and plates. Several 
studies (e.g. Baumgardner and Gandrud (1998)) have established that contrails are initially formed with spherical ice crystals.

Schröder et al. (2000), could not establish a specific shape, reporting an amorphous, near-spherical ice crystal shape. Quasi-spherical ice crystals were reported in young contrails by Meyer et al. (2002), with non-spherical ice shapes in aged contrails.

Table 2.1: Habits of ice crystals determined by in situ contrail studies

\begin{tabular}{|c|c|c|c|c|c|}
\hline Author(s)-year & Shapes & $\mathrm{T}^{\circ} \mathrm{C}$ & range $(\mu \mathrm{m})$ & $\mathrm{N}\left(\mathrm{cm}^{-3}\right)$ & Age \\
\hline Parungo, 1995 & hollow columns & $-25--53$ & $<150$ & & $3 \min$ \\
\hline Sassen, 1997 & spheres & -62 & $10-30$ & $1-8$ & $30-60 \mathrm{~min}$ \\
\hline Baumgardner et al., 1998 & spheres & -63 & $0.4-10$ & $1-1000$ & $25-35 \mathrm{~s}$ \\
\hline Goodman et al., 1998 & $\begin{array}{l}\text { hexagonal plates } \\
\text { columns } \\
\text { triagonal plates }\end{array}$ & -61 & $>0.5$ & $5-10$ & $1 \mathrm{~min}$ \\
\hline Heymsfield et al., 1998 & bullet rosettes & -52 & $1-250$ & $10-100$ & $>60 \mathrm{~min}$ \\
\hline Lawson et al., 1998 & $\begin{array}{l}\text { spheroids } \\
\text { plates } \\
\text { columns } \\
\text { bullet rosettes }\end{array}$ & -55 & $1-600$ & & $40 \mathrm{~min}$ \\
\hline Liou et al., 1998 & $\begin{array}{l}\text { bullet rosette } \\
\text { hollow columns } \\
\text { plates }\end{array}$ & -61.1 & $1-90$ & & $50 \mathrm{~s}$ \\
\hline Sassen and Hsueh, 1998 & spheroids & -57 & $20-30$ & & $>60 \mathrm{~min}$ \\
\hline Spinhirne et al., 1998 & spheres & & $<40$ & & $>6 \mathrm{~h}$ \\
\hline Poellot et al., 1999 & spheres & $-47--66$ & $<50$ & $\sim 100$ & $3-15 \min$ \\
\hline Schröder et al., 2000 & close to spheres & $-53--57$ & $1-10$ & $>100$ & $>30 \min$ \\
\hline Meyert et al., 2002 & spheres & -48 & & & \\
\hline
\end{tabular}

The non-sphericity of the ice crystals reported during the evolution of contrails was presented by Parungo (1995) who reported contrails formed by hollow columns; Goodman et al. (1998) with in situ sampling found that the habits were predominately hexagonal plates $(75 \%)$, columns (20\%) and triangles (5\%). In precipitating contrails at high relative humidity with respet to ice $R H_{\text {ice }}$, Heymsfield et al. (1998) found well-defined bullet rosettes; Lawson et al. (1998), in the study of contrails evolv- 
ing into cirrus clouds, observed that the most common shapes in contrails and cirrus clouds are irregular spheroids, plates, columns and bullet rosettes; and Liou et al. (1998), after obtaining the measurement of the ice crystal size distribution in contrail cirrus, proposed the combination of bulllet rosettes (50\%), hollow columns (30\%) and plates (20\%). The wide variability of ice crystal shapes observed in in-situ studies (table 2.1) constitutes the motivation of this dissertation.

The size range of ice crystals is an important microphysical characteristic in contrails, and has been found to be variable (see table 2.1). In situ observations by Goodman et al. (1998) reported in 1 min young contrail ice crystals in the range $>0.5 \mu \mathrm{m}$. Schröder et al. (2000) has established that young contrails with more than 30 min old have a higher concentration of ice crystals than cirrus clouds, since contrails are formed by smaller ice crystals with mean radius in the range of $1-10 \mu \mathrm{m}$.

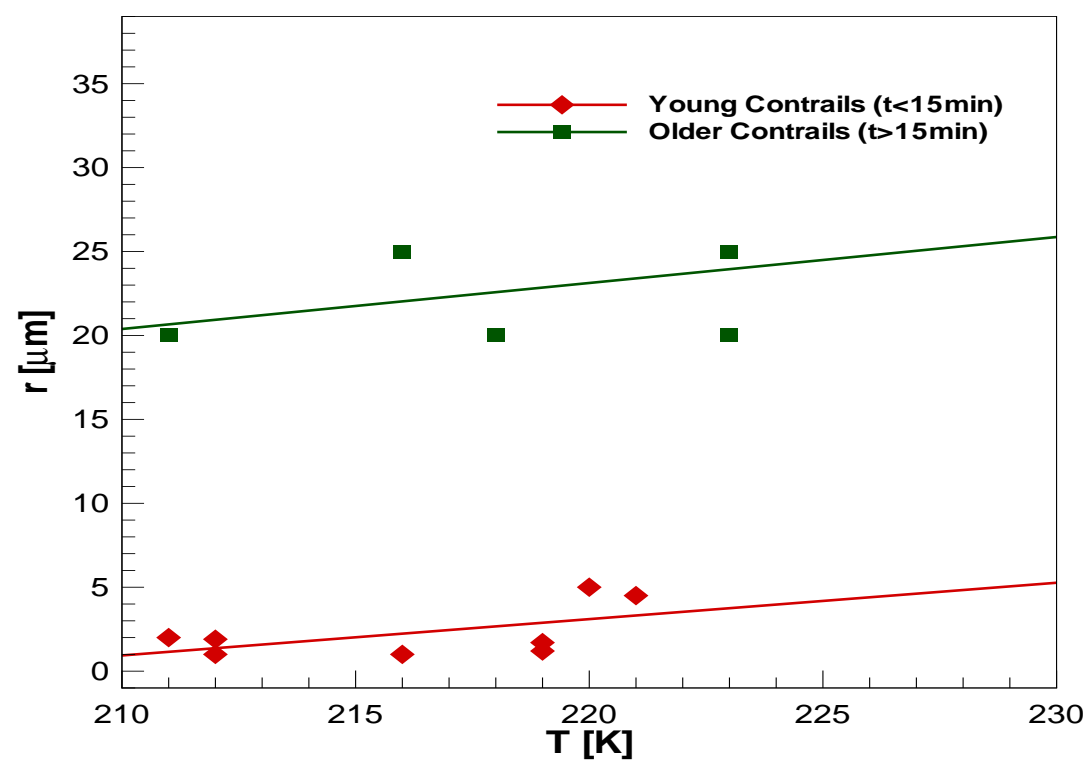

Figure 2.1: Ice particles size - radius and temperature

Figure 2.1 shows the in situ values of the ice particle radius with respect to the 
temperature and time. It is based on all studies presented in table 2.1. The difference in crystal sizes between young and older contrails is clearly observed.

Figure 2.2 shows that favorable atmospheric conditions (humid air and low temperature) permit contrail ice crystals to grow in size, shifting the spectrum toward the largest sizes. Figure 2.3 shows that, as a consequence of the growth process the aged persistent contrails appear to have similar particle sizes as young cirrus (Schröder et al., 2000). Poellot et al. (1999) found small crystals $(<50 \mu \mathrm{m})$; the study of contrails evolving into cirrus clouds by Heymsfield et al. (1998) reported sizes in the range 1-250 $\mu \mathrm{m}$. However, the largest range observed $(1-600 \mu \mathrm{m})$ was reported by Lawson et al. (1998).

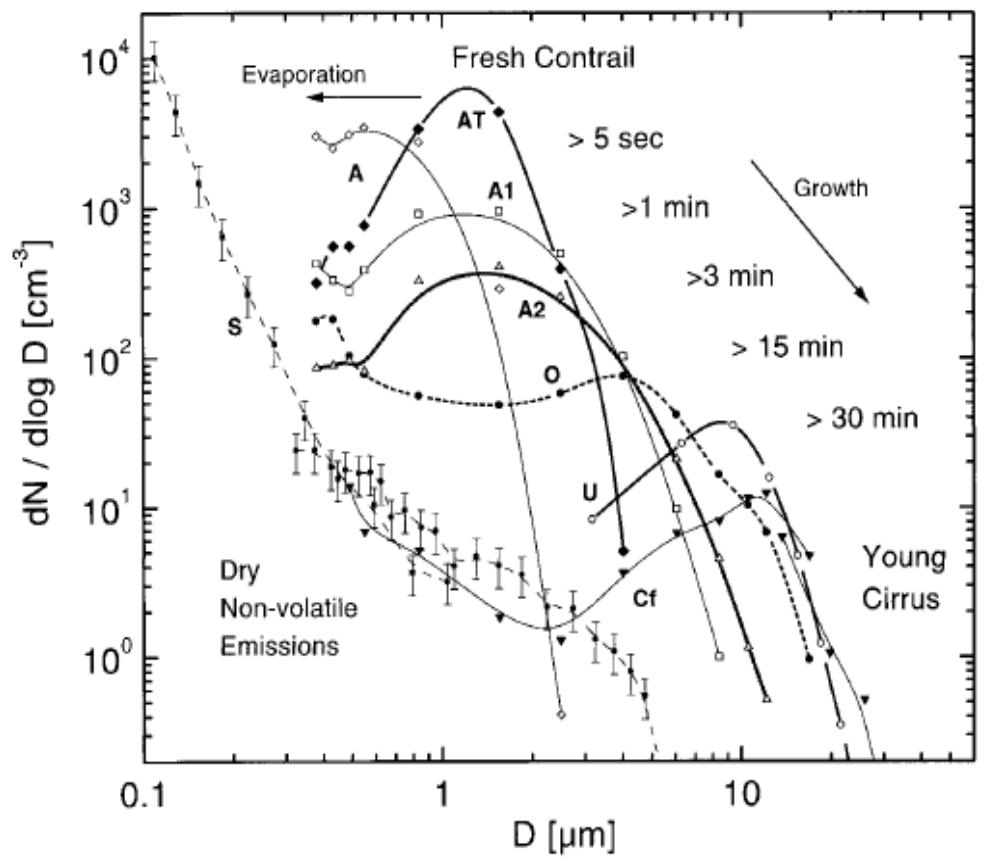

Figure 2.2: Ice particle size spectra in contrails of different ages at ranges temperature [215K-219K] and relative humidity [30\%-80\%]. Adapted from (Schröder et al., 2000)

The typical concentration of ice crystals in young contrails (age $<1 \mathrm{~min}$ ) is high, exceeding a few $1000 \mathrm{~cm}^{-3}$, but aged contrails present an important decrease in the concentration by two or three orders of magnitude (Spinhirne et al., 1998; 
Schröder et al., 2000; Heymsfield et al., 1998).

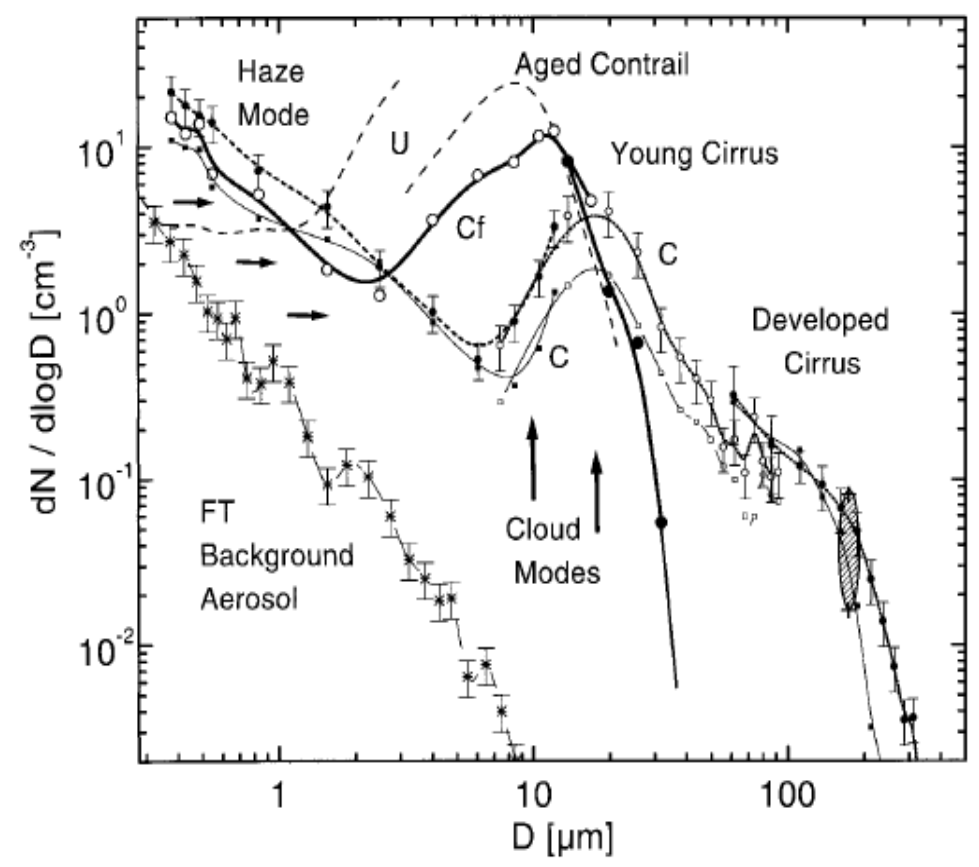

Figure 2.3: Ice particle size spectra in young cirrus clouds at ranges [213K-233K] and relative humidity [60\%-85\%]. Adapted from (Schröder et al., 2000)

\subsection{Definition of ice crystal shapes}

Differences in ice crystal shapes depend on temperature, water vapor supply (supersaturation with respect to ice) and time. Magono and Lee (1966) established that there are up to 80 ice crystal habits that could be formed in the atmosphere.

The recent laboratory study by Bailey and Hallett (2004) suggested a summary of habits (figure 2.4) that are in agreement with the complex habits found in situ observation of contrails.

The shapes of ice crystals assumed in the numerical simulation in this study are based on the shapes observed in contrails (see table 2.1). Libbrecht (2005) reported some of these ice crystals shapes (figure 2.5). A simple plate-like, a simple hollow 


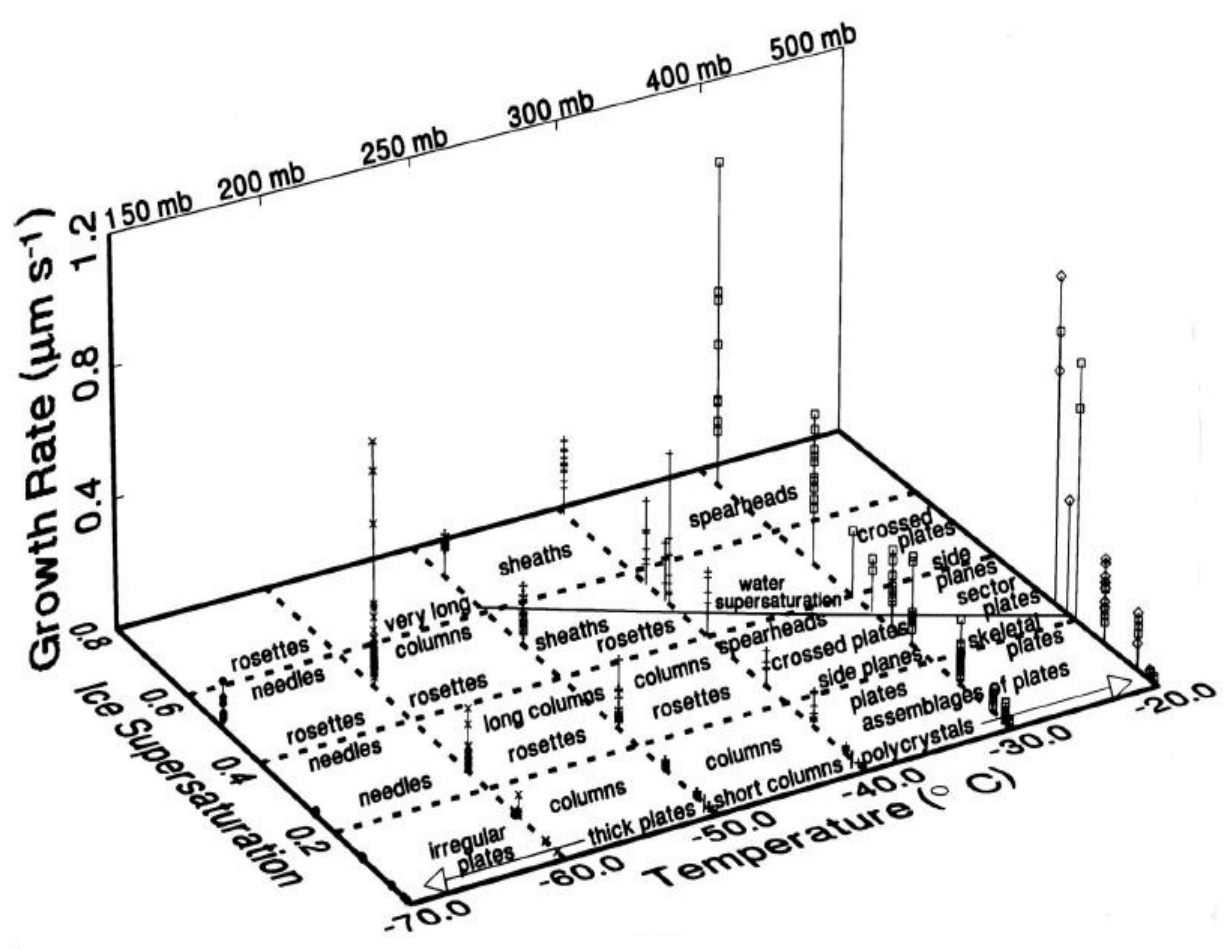

Figure 2.4: Summary of ice habits between $-20^{\circ} \mathrm{C}$ and $-70^{\circ} \mathrm{C}$ courtesy of Bailey and Hallett (2004)

hexagonal columnar, a needle-like and a simple four rosette bullet ice crystals are shown.

The ice crystals can be solid or hollow, but for our study they will be considered solid. Every shape is complicated to represent in a simulation. An important simplification is based on the fact that ice crystals are often axially symmetric, such that their shapes can be described by some characteristic dimensions (i.e., length along axis, width and the aspect ratio), or alternatively, their shapes can be approximated by spheroids (oblate and prolate) (MacDonald, 1963). The dimensional values are directly involved in shape coefficients or parameters (such as capacitance, ventilation factors, Kelvin effect, and fall velocity) that strongly influence the diffusional growth and precipitation process.

The ice crystals shapes implemented were defined by Bailey and Hallett (2004) as 

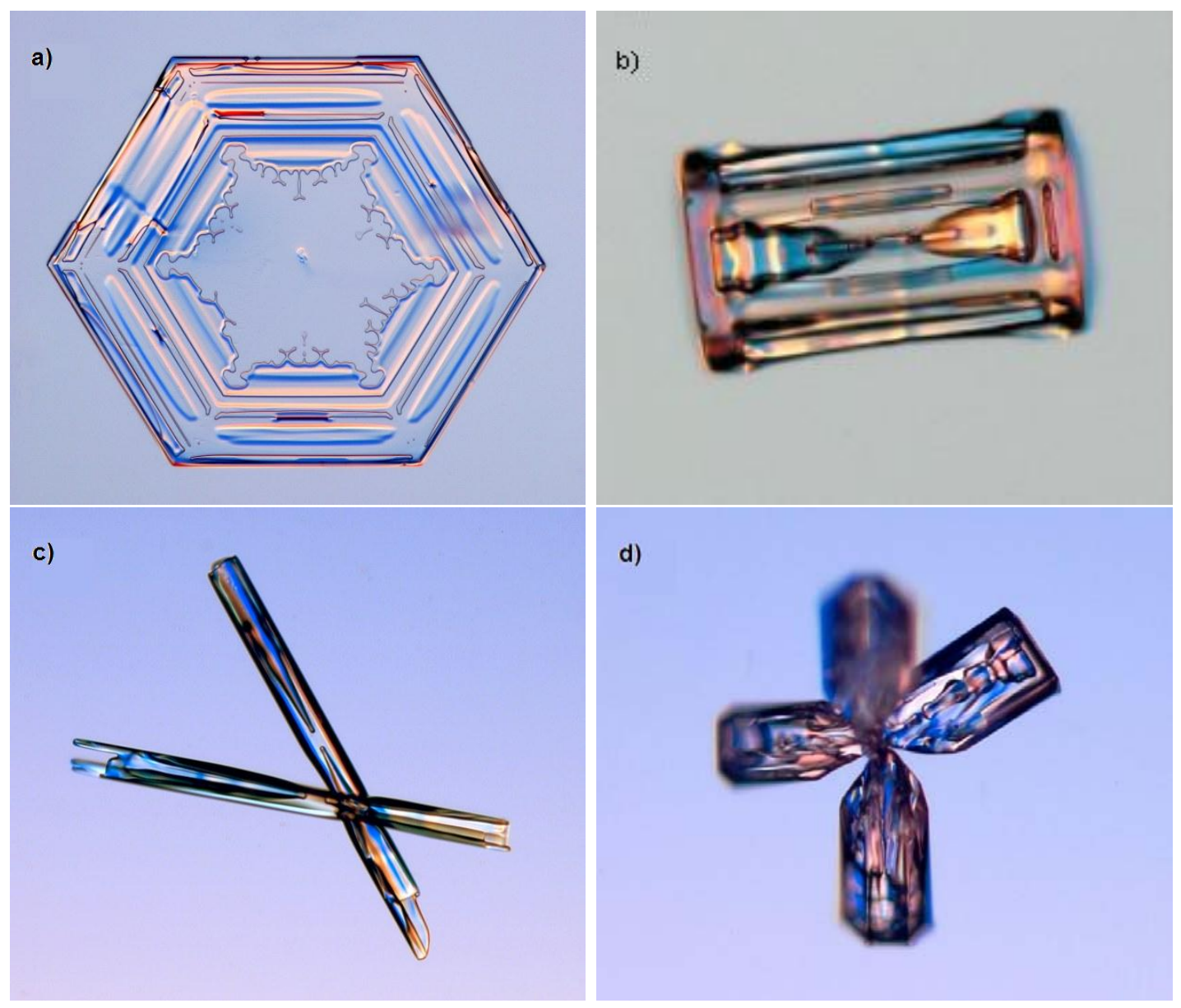

Figure 2.5: Examples of several different morphological types of ice crystals. a) A relatively simple plate-like crystal, b) A simple hollow hexagonal columnar, c) Needlelike crystals and d) A simple four rosette bullet crystal, courtesy (Libbrecht, 2005)

follows:

\subsubsection{Hexagonal Columns:}

These ice crystals consist of two simple hexagonal faces and lengths (L) greater than basal face widths $(\mathrm{w})$. The other characteristic lengths are side of hexagon (s) and the apothem (h) (figure 2.6). In the case of hexagonal ice crystals the dimensions are calculated using a geometrical relation called aspect ratio. 


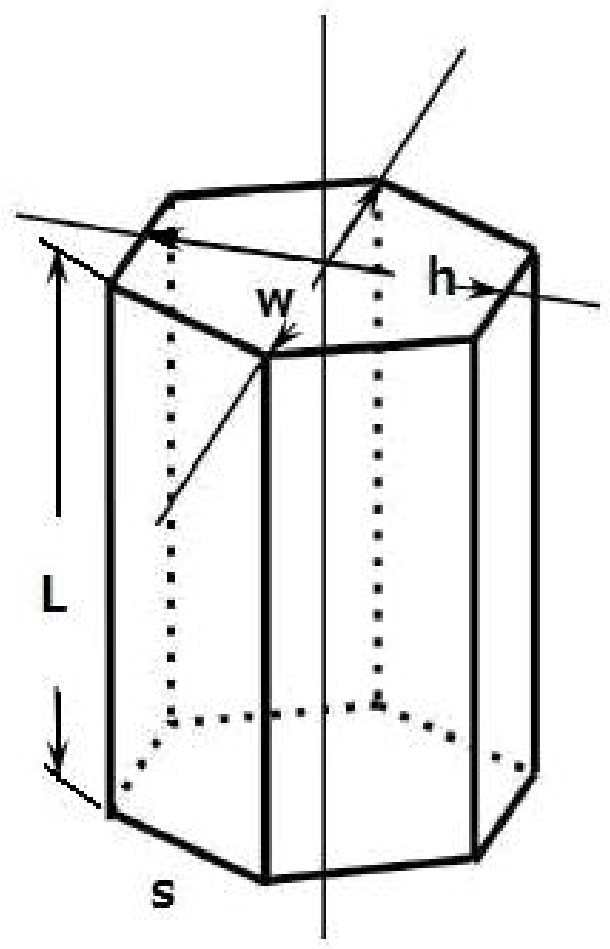

Figure 2.6: Schematic representation of the hexagonal column and plate shapes

\subsubsection{Hexagonal Plates:}

These have the same characteristics as hexagonal columns, but with basal face width (w) larger than prism face height (L).

\subsubsection{Aspect ratio $A r$}

For a hexagonal column and plate with height $(\mathrm{L})$ and basal face width $(w)$ (2 times the side length of the hexagon), the aspect ratio is defined as $A r=L / w$. For hexagonal columns $A r \geq 1$, while for hexagonal plates $A r \leq 1$. 


\subsubsection{Bullet Rosettes:}

These are polycrystals formed by bullets (hexagonal columns) radiating from a common growth center. The single bullet is formed by a hexagonal column and a pyramidal end (figure 2.7).
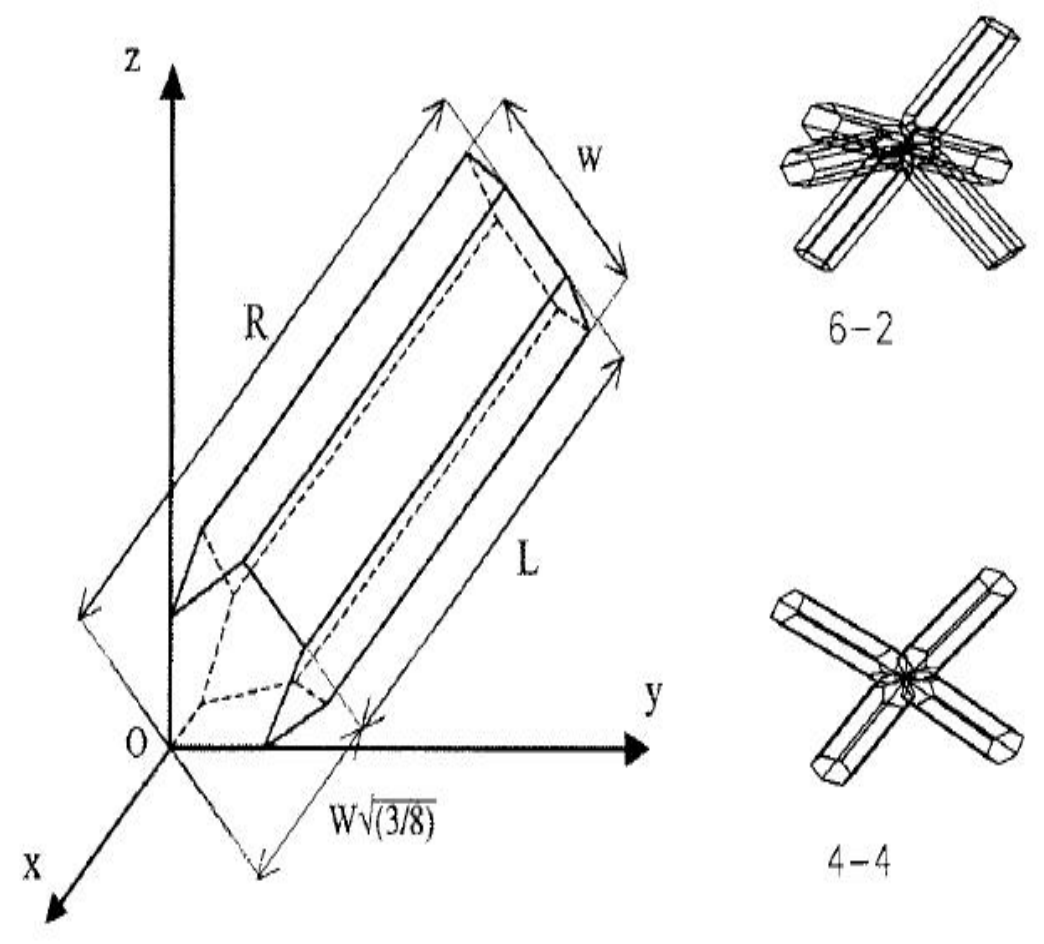

$4-4$

Figure 2.7: Schematic representation of the bullet rosette shape, the elementary single bullet and configuration 6-2 and 4-4 from (Heymsfield and Iaquinta, 2000a)

The bullet rosette shape assumed herein was used by Heymsfield et al. (1998) and Lawson et al. (1998). Heymsfield et al. (1998) found this shape in highly supersaturated ambient with respect to ice $\left(R H_{\text {ice }}=120 \%-140 \%\right)$, while in the same year Lawson et al. (1998) found this shape in supersaturated ambient, defining that ice crystals with lengths larger than $200 \mu \mathrm{m}$ are definitively bullet rosettes, similar to what is observed in natural cirrus. 


\subsection{Radiation}

Radiation studies on contrails have been performed in two areas: 1) effects of solar radiation related on contrail evolution and 2) effects of contrail on solar radiation. This study has the interest to determine the radiation effects on contrail dynamics and the possible influence of the crystal shape.

The lasting effects of contrails include their impact on the radiation balance of the Earth. The evolution of persistent contrails in the atmosphere involves radiative processes that contribute to net cooling/negative forcing [by reflecting the incident solar radiation (Shortwave (SW) 0.2-5 $\mu \mathrm{m}$ ) or heating/positive forcing by absorbing infrared radiation, reducing the outgoing (Longwave (LW) 5 - $50 \mu \mathrm{m}$ ) emitted to space. Studies on contrail evolution with spherical ice crystals and coupled with radiation reported that small infrared cooling rate leads to minimal changes in the contrail dynamics (Chlond, 1998). Gierens (1996) found a similar behavior of contrails (hexagonal columns) coupled with solar and infrared radiation. However, Jensen et al. (1998) observed significant updraft dynamics by a net heating on contrails (spheres). The heating process is mainly driven by the absorption of infrared radiation emitted by the Earth's surface. An increase in altitude increases the relative humidity through adiabatic cooling. The higher supersaturation generated by the updraft dynamics can be important for the shape effect studies of older contrails.

In situ contrail studies have established that the number density of ice crystals is much larger than in cirrus clouds, but with smaller size spectrum (Schröder et al., 2000). The contrail optical depth is a function of crystal shape, the size spectrum and ice water path (IWP). This optical depth determines how much longwave radiation is either absorbed or emitted and how much short wave radiation is absorbed (Yang et al., 2008). The crystal size is an important parameter in contrail absorption and reflection. Contrails with high concentration of small ice crystals will reflect 
more solar radiation. This is because the small ice crystals have larger cross-sectional area given fixed IWC. Studies on contrails and cirrus clouds reported that small ice particles produce strong heating rates depending on IWP (Ebert and Curry, 1992; Meerkötter et al., 1999). The shape effect plays an important role in the absorption and reflection. For a given ice crystal size, the cloud reflection decreases in the following shape order: bullet rosettes, hollow columns, plates and spheres because the increasing asymmetry allows strong forward scattering Liou et al. (1998).

\subsection{Ice Deposition Coefficient $\beta_{i}$}

Studies on cirrus evolution found that the ice deposition coefficient is a critical parameter to determine the total number of ice crystals. In homogeneous nucleation, this parameter affects mainly the growth rate of smaller ice crystals. The number of ice crystals decreases as $\beta_{i}$ increases (Lin et al., 2002). Laboratory studies by Haynes et al. (1992) reported possible extreme values in the interval 0.001-1. Magee et al. (2006) found by laboratory measurements the value of 0.006 .

Lohmann et al. (2008) found that a decrease of $\beta_{i}$ from 0.5 to 0.006 as was suggested by laboratory measurement leads to an increase in the ice crystal number concentration that is not realistic. A recent study of the influence of ambient supersaturation and ice crystal size on deposition coefficient was reported by Nelson and Kay (2007). Ice crystal deposition coefficient affects ice crystal habit (shape and size), which are directly related to cloud processes and cloud radiative impacts.

An important parameter in contrail simulations is the number of ice crystals. The concentration level of ice crystals remaining in young contrails related to the size range can influence the lasting effects of older contrails. As the ice crystal number is potentially sensitive to the deposition coefficient and the latter is so uncertain it is necessary to include its analysis in studying the evolution of persistent contrails into 
contrails-cirrus.

This brief background helps us to have a more realistic level of understanding about the habits found in contrails and the influence on radiation impact of contrails. They are not formed strictly by a specific shape of ice crystal; instead there is a combination of shapes inside of the plume. A modest attempt to simulate contrails close to reality requires the implementation of a combined ice spectrum formed by hexagonal columns and bullet rosettes and an initial study of shape effect coupled with radiation. The next chapter treats all sets of equations and assumptions taken to implement every shape individually, followed by a combined ice spectrum. 


\section{Chapter 3}

\section{Numerical Model}

The evolution of contrails involves atmospheric flows influenced by different scales of turbulent motions. This nonlinear phenomenon is complicated to study theoretically, therefore numerical simulations are most often used to investigate it. In atmospheric flows the Reynolds numbers are sufficiently large that direct numerical simulation (DNS) of the solution of the Navier-Stokes equations is not practical. Large-eddy simulations (LES), where the Navier-Stokes equations are solved directly for the most important scales of motion and the effects of turbulence from the smallest scales are modeled become the most realistic feasible solution method.

\subsection{Model Description - Fluid Dynamics}

The Large-Eddy Simulation code that forms the core of the WVU contrail code has been used during the last years in different turbulent phenomena in the atmosphere such as tornadoes (Lewellen et al., 1997, 2000), and boundary layer cloud modeling (Lewellen and Lewellen, 1998, 2002).

It was derived from an LES code developed primarily for boundary layer cloud modeling as described in Sykes and Henn (1989); Sykes et al. (1990). Finite difference in its three-dimensional scheme, it was used to model the incompressible Navier-Stokes 
equations with the Boussinesq approximation for stretched and staggered grids with an accuracy of second-order in time and space. In the study of aircraft wakes, it has been used to simulate the vortex pair breakup and the accompanying exhaust dispersion in the atmosphere (Lewellen and Lewellen, 1996), compared with Lidar measurements (Lewellen et al., 1998), favorably used to model the effects of wake dynamics on chemistry within the wake vortex (Lewellen and Lewellen, 2001b) and on contrail evolution studies out to 30 minutes (Lewellen and Lewellen, 2001a).

The initial stage in the present simulation reads the wake results from the 2-D Boeing data at one second after the exhaust gases of the B-767 aircraft have left the engines. This data is obtained by a combination of in-situ measurement and numerical simulations. The ice crystals, engine water and heat are distributed in the grid proportionally to the concentration of a passive exhaust tracer. Additional simulations are required to generate an ambient turbulent field that will be added to the simulation. At this point the contrail can be simulated.

Contrail development at early times was described in four overlapping regimes by Lewellen and Lewellen (2001a):

In the evolution of an aircraft contrail, it is convenient to identify four overlapping regimes. During the first few seconds, the engine exhaust jets rapidly mix with ambient air and, for suitable atmospheric conditions, become supersaturated with respect to water and form a contrail (see, e.g., Schummann (1996)). During the same period, the vorticity shed from the wings rolls up into a pair of trailing vortices. The wake dynamics following this roll-up/jet regime are dominated by the interactions of the vortex pair. Typically in this stage the engine exhaust jets partly wrap into the vortex cores and partly detrain into a buoyant plume; the vortices fall and interact with each other through a mutual induction sinusoidal 
instability (Crow, 1970) and with any ambient shear that is present, until they finally break up, typically within a few minutes. Given ambient conditions supersaturated with respect to ice, the contrail can grow as moist air mixes with the ice crystal-rich exhaust plume. After vortex breakup, positive buoyancy acquired from the hot engine exhausts and from the vortex pair falling through any ambient stratification can dominate the dynamics until the plume mixes sufficiently with the ambient air, typically within one or two BruntVäisälä periods ( 10-20 min). Finally, at later times, the plume dispersion is dominated by the interaction with the ambient atmosphere via atmospheric turbulence, gravity waves, and shear. The latent heat release and radiative cooling within the contrail may also contribute significantly at this point as these effects are no longer dwarfed by the wake-induced dynamics. Under favorable conditions (particularly a large ambient supersaturation with respect to ice), the ice mass in the contrail can continue to grow and persist until the individual ice crystals become heavy enough to precipitate out.

\subsection{Model Description - Ice Microphysics}

There are, in general, two distinct approaches to handling the ice microphysics involved in the contrail evolution models: bulk and binned. The bin-resolving microphysics model provides more rigorous solutions than the bulk model; this is because the bulk microphysics approach is a relatively simple model, assuming a fixed spectrum, losing the information about higher moments of the particle size distribution within the cell, and therefore the computational cost is significantly lower than binned microphysics (Lewellen and Lewellen, 2001a). The physical effects included in both microphysics models in the WVU code are diffusional growth/sublimation, sedimen- 
tation, buoyancy due to latent heat release, and perturbation pressure effects.

The binned microphysics treatment used in the WVU contrail code was described in detail by Lewellen et al. (2009). The ice population during the evolution of a contrail varies in location and time. Young contrail is characterized by high population of small ice crystals in the core and low population of large crystals in its periphery. This means that during a simulation is only used a fraction of the total ice bin spectrum. The contrail code includes this major modification through the "dynamic local binning" reducing in output file size, memory usage and computer time consuming. This improvement defines a "global ice bin space" as the total number of ice bins that form the spectrum, which is discretized and divided into a number of sections (particle-size bins) with a mass ratio value between bins, having defined a smallest ice mass. The required fraction of the whole ice bin space is handle using a sub-space called "local ice bin space" with a fixed bin number that can be less or equal than the "global ice bin space".

A study by Huebsch and Lewellen (2006a) compared bulk and binned results at two different ambient supersaturation levels with respect to ice $\left(R H_{i c e}=110 \%\right.$ and $130 \%$ ). Figure 3.1 shows the contrail metric of total ice crystal number per meter of flight path, $\mathrm{N}(\mathrm{t})$, that permits one to follow the evolution of the total ice particles in the plume. The number of ice crystals lost at early times is governed by the adiabatic compressional heating and the mixing process. The adiabatic compression and heating are a consequence of the descending vortices, causing sub saturation and evaporation of the ice (Sussmann and Gierens, 1999; Lewellen and Lewellen, 2001a). After the wake vortex has died completely, the evolution of the ice crystal number depends on the mixing due to atmospheric turbulence and the supersaturation level.

The results show a dramatic difference in the evolution of ice crystal number distribution with time for the bulk and binned models. The ice crystals lost are never 


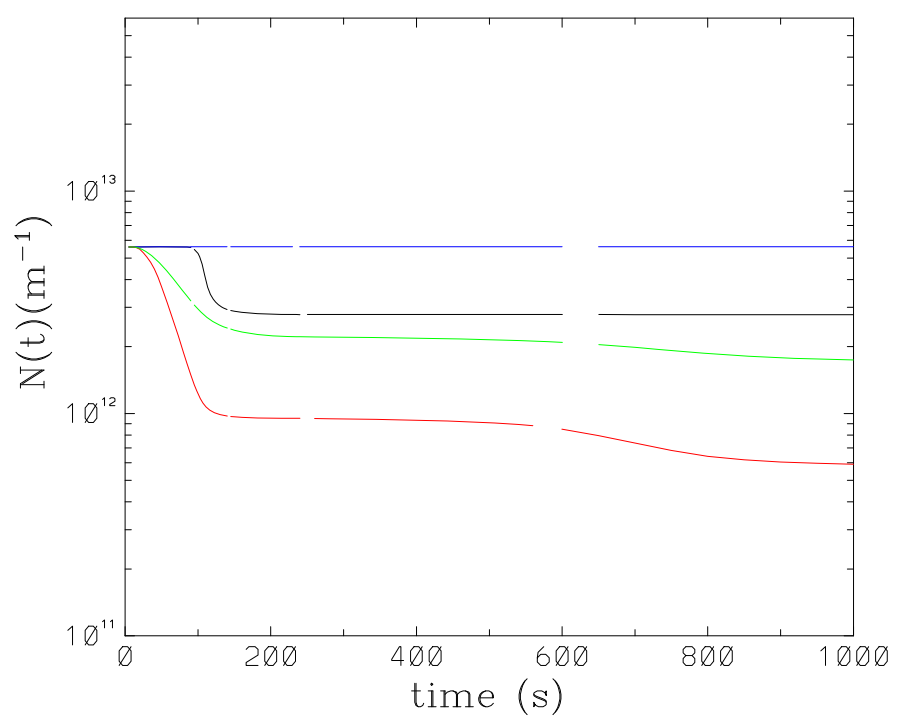

Figure 3.1: Changes in total ice crystal number as a function of time for Boeing platform B-767 at baseline conditions: stable stratification given by a potential temperature gradient of $2.5 \mathrm{~K} / \mathrm{km}, P_{\text {ref }}=250 \mathrm{hPa}$ and $T_{r e f}=220 \mathrm{~K}$, with $R H_{\text {ice }}=$ $110 \%$ (bulk ice: black; binned ice: red) and $R H_{\text {ice }}=130 \%$ (bulk ice: blue; binned ice: green)

recovered, and this leads to significant differences in contrail properties (e.g., optical depth or mean crystal size) that will persist in time. The ice crystals lost during the falling vortices system and the late-time crystal loss due to buoyant oscillations of the plume are only captured with more detail in the binned microphysics model.

This analysis and comparison of the results between the bulk and binned microphysics shows that the the binned model is the most appropriate to simulate the evolution of contrails because this model captures more physical details at early and later time.

\subsection{Binned Ice Microphysics}

The implementation of additional ice crystals shapes in the WVU contrail code through CARMA routines requires adding or modifying some routines to cover the 
spherical, hexagonal columns, bullet rosettes and a combination of shapes.

The rate of mass growth of a single ice crystal is handled at a level similar to that of Ackerman et al. (1995). The modification in the equation is very simple, the capacitance for the spherical shape is the radius " $r$ " and is changed by a capacitance value "C" of each shape (including spheres).

$$
\frac{d m_{i}}{d t}=\frac{g_{0} e_{s x}\left(S+1-A_{k}\right)}{1+g_{0} g_{1} e_{s x}}
$$

where the various coefficients are:

Coefficient for particle without radiation or heat conduction:

$$
g_{0}=\frac{4 \pi C D f_{v} m_{v}}{R T}
$$

Coefficient for heat conduction:

$$
g_{1}=\frac{m_{v} L_{h}^{2}}{R T^{2} \lambda f_{t} 4 \pi C}
$$

Saturation vapor pressure (hPa), from (Laube and Höller 1986) via Chlond (1998).

$$
e_{s x}=\exp \left(\frac{-6147.795166}{T}+24.31720686\right)
$$

Water vapor supersaturation

$$
S=\frac{q_{t}-q_{i}}{q_{s i}}-1=\frac{q_{v}}{q_{s i}}-1=\left(R H_{i c e} / 100\right)-1
$$

\subsubsection{Kelvin Effect}

The factor $A_{k}$ in equation 3.1 accounts for the increase in vapor pressure exerted by the ice crystal due to the Kelvin curvature effect. The Kelvin curvature effect is 
given by equation 3.6 which is a function of temperature, density, surface tension and characteristic length.

$$
A_{k}=\exp \left(\frac{2 m_{v} \sigma_{i / v}}{R T \rho_{i} \delta}\right)
$$

The variables that are directly related to the shape of the ice crystals are the surface tension $\sigma_{i / v}$ and the characteristic length $\delta$. For spherical ice particles the surface tension at ice/vapor interface is handled by the expression $\sigma_{i / v}=141.0-0.15 *$ T(K), (Hale and Plummer, 1974). However, for hexagonal columns, plates and bullet rosettes an equation that expresses the temperature dependence for the surface tension at ice/vapor interface $\sigma_{i / v}$ is not available in the literature. Pruppacher and Klett (1997) cite for hexagonal columns two values for the $\sigma_{i / v}$ at $273 \mathrm{~K}\left(\sigma_{i / v}=104 \mathrm{erg} /\right.$ $\left.\mathrm{cm}^{2}\right)$ and at $233 \mathrm{~K}\left(\sigma_{i / v}=106 \mathrm{erg} / \mathrm{cm}^{2}\right)$. In order to have an expression for the surface tension, we have followed the same procedure as Fortin et al. (2003). A straight line was fit with the two data points available in the literature, obtaining the final expression: $\sigma_{i / v}=117.658-0.05 * T(\mathrm{~K})$.

Pruppacher and Klett (1997) define the characteristic length $\delta$ as the distance of the face to the center of the ice particles. For the case of spheres this is the radius "r"; for hexagonal columns and plates the apothem " $h$ ", that is variable of each bin size, with dependence on the aspect ratio, defined by Spichtinger and Gierens (2008) in equation 3.7 as discussed subsequently in this chapter. In the case of bullet rosettes every bullet is a single hexagonal column (with different aspect ratio), and hence the characteristic length is the apothem of a single bullet.

The term $A_{k}$ for different ice crystal shapes as a function of mass (or radius) is presented in figure 3.2. For the mass range shown the spheres had radii in the range of 0.05 to $163 \mu \mathrm{m}$, while the hexagonal columns, hexagonal plates and bullet rosettes (6 and 4 bullets) with apothems in the range of 0.043 to $64.9 \mu \mathrm{m}, 0.02$ to $35.2 \mu \mathrm{m}$, 


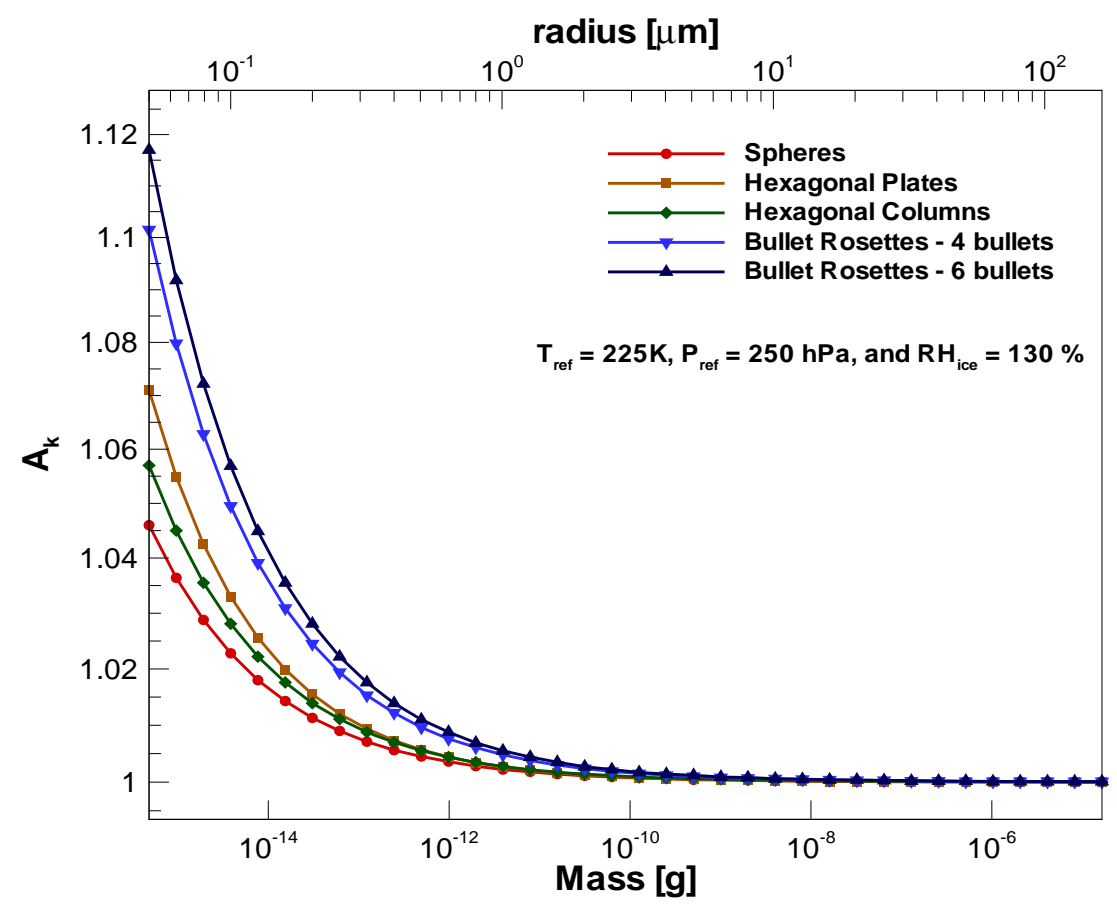

Figure 3.2: Kelvin effect term for different ice crystal shapes: spheres, hexagonal columns and bullet rosettes

0.033 to $202 \mu \mathrm{m}$, and 0.023 to $40.3 \mu \mathrm{m}$ respectively. The Kelvin curvature effect is largest for bullet rosette since its characteristic length is smaller than that of any other shapes having the same crystal mass.

\subsubsection{Aspect Ratio and Shape Dimensions}

In this study the aspect ratio $A r$ for hexagonal columns (equation 3.7) is taken as a function of ice crystal mass from Spichtinger and Gierens (2008), with a different ice density $\rho_{i}=0.917 \mathrm{~g} / \mathrm{cm}^{3}$. This relation involves the volume formula of a hexagonal column and the mass-length relations (parameters $\alpha$ and $\beta$ ) from 
Heymsfield and Iaquinta (2000a).

$$
A r= \begin{cases}1 & , m_{i}<m s h_{\text {limit }} \\ \sqrt{\frac{\sqrt{27} \rho_{i}}{8 \alpha^{\frac{3}{\beta}} m_{i}^{\frac{3-\beta}{2 \beta}}}} & , m_{i} \geq m s h_{\text {limit }}\end{cases}
$$

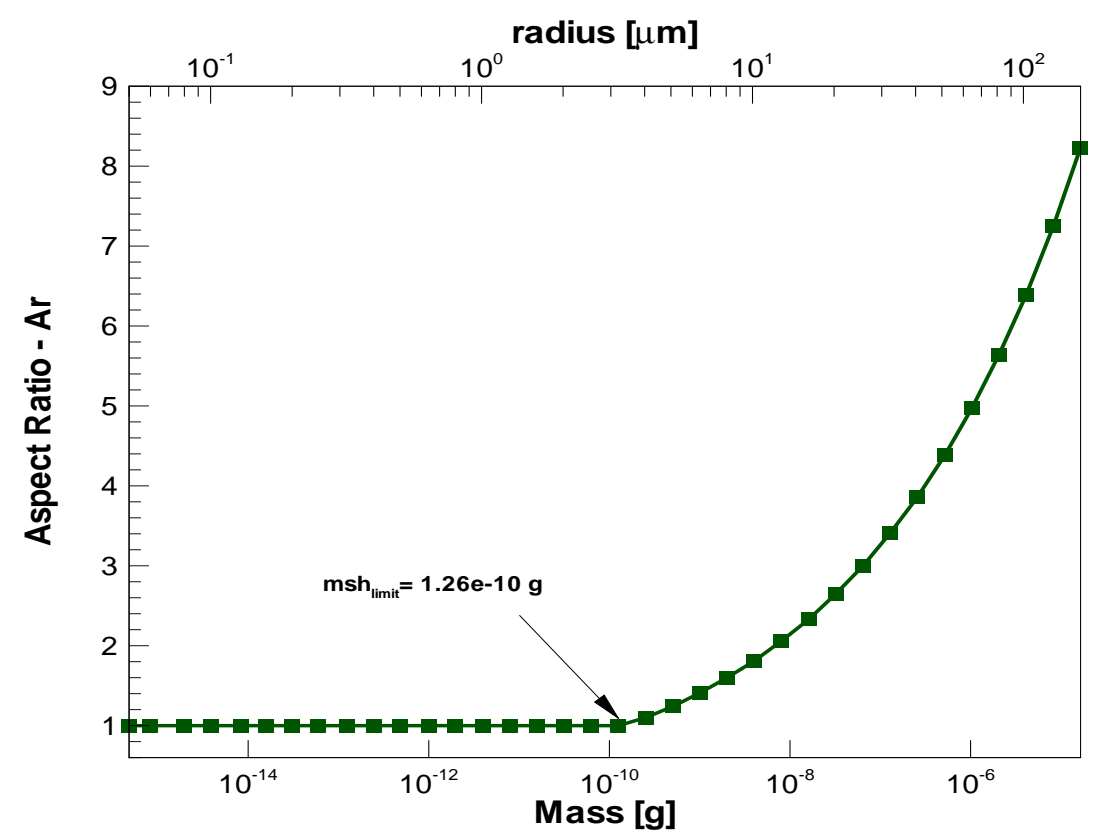

Figure 3.3: Aspect ratio of hexagonal column crystals

The ice spectrum is divided in two groups of particles (figure 3.3), with the smaller with aspect ratio equal to 1 , being close to spherical, supported by Lawson et al. (1998) who established that small ice crystals in contrails appear to have spheroidal shape. The rest of the ice crystals have aspect ratios bigger than 1 (with a maximum value of 8.23), giving a good approximation to the size distribution of in situ observations. This assumption is reasonable since it is known that contrail ice crystals can have different shapes and hexagonal columnar is among the most common observed in contrails (Lawson et al., 1998; Parungo, 1995). The limit between small and large particles is established by the parameter ice mass $m s h_{\text {limit }}=1.26 \times 10^{-10}$ 
g or equivalent length $3.2 \mu \mathrm{m}$ as was used by Spichtinger and Gierens (2008).

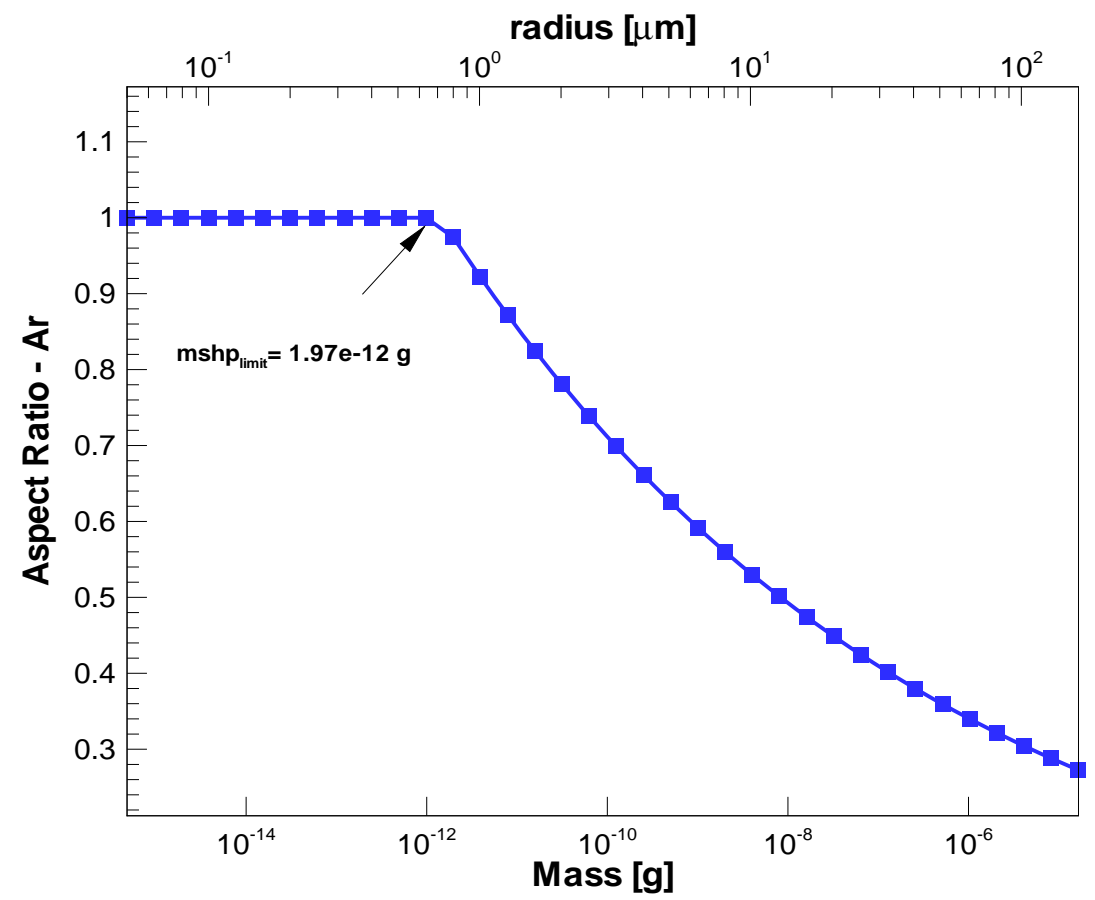

Figure 3.4: Aspect ratio of hexagonal plate crystals

The solid hexagonal plate also was characterized by aspect ratio. It was defined as in the hexagonal columns. This relation was handled as a function of mass using relations of Pruppacher and Klett (1997). This involves the volume formula of a hexagonal plate and dimensional relationships of thickness and diameter.

$$
A r= \begin{cases}1.0 & m_{i}<m s h p_{\text {limit }} \\ 0.1130 m_{i}^{-0.0799} & m_{i} \geq m s h p_{\text {limit }}\end{cases}
$$

The mass limit between small and large particles is defined as $m s h p_{\text {limit }}=1.97 \times$ $10^{-12}(\mathrm{~g})$ or equivalent length $0.63 \mu \mathrm{m}$. As in the hexagonal columns the spectrum is divided in two groups of particles (figure 3.4 ), the smaller with aspect ratio equal to 1, close to spherical. However, the large particles have an aspect ratio less than 1. 
The bullet rosette ice crystal dimensions are calculated based on the mathematical relations used in Heymsfield and Iaquinta (2000a). The dimensions of each bullet are a function of the aspect ratio, that is calculated as in the hexagonal column shape. The capacitance of a bullet rosette shape also needs the calculation of two dimensional parameters:

\section{Length of a single bullet $L_{b}$}

The characteristic length of a single bullet is a dimension calculated as a function of ice mass, ice density and aspect ratio. The relation was taken from equation A11 from Heymsfield and Iaquinta (2000a).

$$
L_{b}=\left(\frac{m_{i}}{n_{b} \rho_{i}\left(0.65+0.21 A r^{-1}\right) A r^{-2}}\right)^{1 / 3}
$$

\section{Maximum Dimension $D_{\max }$}

The maximum dimension of a bullet rosette $D_{\max }$ is used to calculate the capacitance of bullet rosette ice crystals. The equation and constants $C_{a}$ and $C_{b}$ only for two configurations (4-4 and 6-2)are given in table 3.1 from Heymsfield and Iaquinta (2000a).

$$
D_{\max }=\left(C_{a}+C_{b} A r^{-1}\right) L_{b}
$$

Table 3.1: Constants for configurations 4-4 and 6-2 to calculate $D_{\max }$

\begin{tabular}{lll}
\hline & $4-4$ & $6-2$ \\
\hline$C_{a}$ & 1.612 & 1.964 \\
\hline$C_{b}$ & 1.566 & 1.390 \\
\hline
\end{tabular}




\subsubsection{Ice Surface Area}

The total ice surface area per length of contrail, $\mathrm{S}(\mathrm{t})$, is an important ice contrail metric that depends on the individual crystal surface areas for each shape. The equations used are:

- Spheres: .

$$
S_{s p h}=4 \pi r^{2}
$$

- Hexagonal Column and Hexagonal Plate: The surface area is calculated by the area of 6 rectangles and 2 hexagonal faces.

$$
S_{h c / h p}=6 s(h+L)
$$

- Bullet Rosette: From figure 2.7 the surface area is calculated in two parts: the hexagonal column part: (1 hexagonal, 6 rectangle faces), the pyramidal end part: (3 pentagons and 3 triangles faces).

$$
S_{b r}=n_{b}[s(4.5 h+6 L+5.1614 s)]
$$

For a given mass the bullet rosette shape has the largest surface area, followed in order by the hexagonal column, hexagonal plate and sphere (figure 3.5).

\subsubsection{Combination of shapes:}

As was discussed previously, contrails are formed by a wide spectrum of ice shapes. The simulation of realistic contrails needs to have an ice spectrum that reflects the ice particles found in in situ observations. The modest combined ice spectrum consists as follows: the smallest particles are considered spherical, the medium-sized hexagonal 


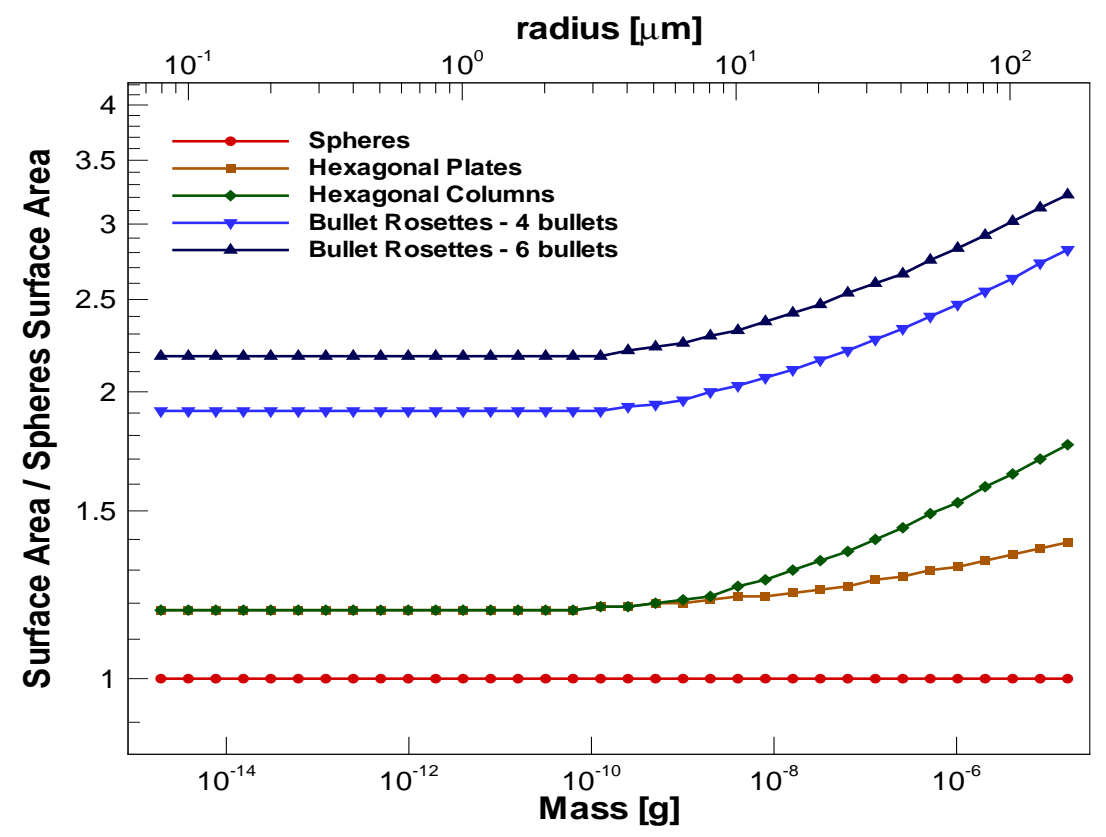

Figure 3.5: Surface area of single ice crystals

columns and the largest bullet rosettes. This initial attempt to recreate the transitional evolution of the ice crystals is an approximation of first order that should be improved in the future. The proposed spectrum follows the habit transition observed in the atmosphere and laboratory (Bailey and Hallett, 2004).

The complex behavior of ice crystal habit is strongly dependent on temperature and ice-supersaturation level. This means that detailed studies of ice crystal habits and transitional processes is needed to have a more realistic spectrum. Hexagonal columns with aspect ratio 1 (close to spheres) and hexagonal column particles have the same limit used by Spichtinger and Gierens (2008) $\left(m s h_{\text {limit }}=2.146 \times 10^{-10}(\mathrm{~g})\right)$. The limit for hexagonal columns and bullet rosettes $\left(m h b_{\text {limit }}=1.8963 \times 10^{-7}(\mathrm{~g})\right)$ was calculated based on the results found by Lawson et al. (1998), where the largest particles $(\geq 200 \mu \mathrm{m})$ are mainly bullet rosettes. 


\subsubsection{Capacitance Factor $C$}

The stationary diffusion shape factor, also called capacitance $C$ (used in equation 3.1), considers the influence of ice shape on the field of water vapor around the crystal. A number of methods have been used to calculate the capacitance factor: MacDonald (1963) and Podzimek (1996) used metal models to simulate ice crystals and measure the corresponding capacitance, while Chiruta and Wang $(2003,2005)$ through the finite-element method calculated the capacitance of bullet rosettes, solid and hollow hexagonal columns. Recently Westbrook et al. (2008), using the random walk method to calculate the capacitance of hexagonal column, plates, bullet rosettes, stellar, dendrites and aggregates ice crystals.

The capacitances of the different ice crystal shapes used in our simulations were taken from Westbrook et al. (2008), with dimensions of length [cm], and are a function of the aspect ratio.

1. Spheres:

$$
C=r
$$

2. Hexagonal Columns and Hexagonal Plates:

$$
C=0.58\left(1+0.95 A r^{0.75}\right) s
$$

3. Bullet Rosettes 4 - bullets:

$$
C=0.35 A r^{-0.27} D_{\max }
$$

4. Bullet Rosettes 6 - bullets:

$$
C=0.40 A r^{-0.25} D_{\max }
$$




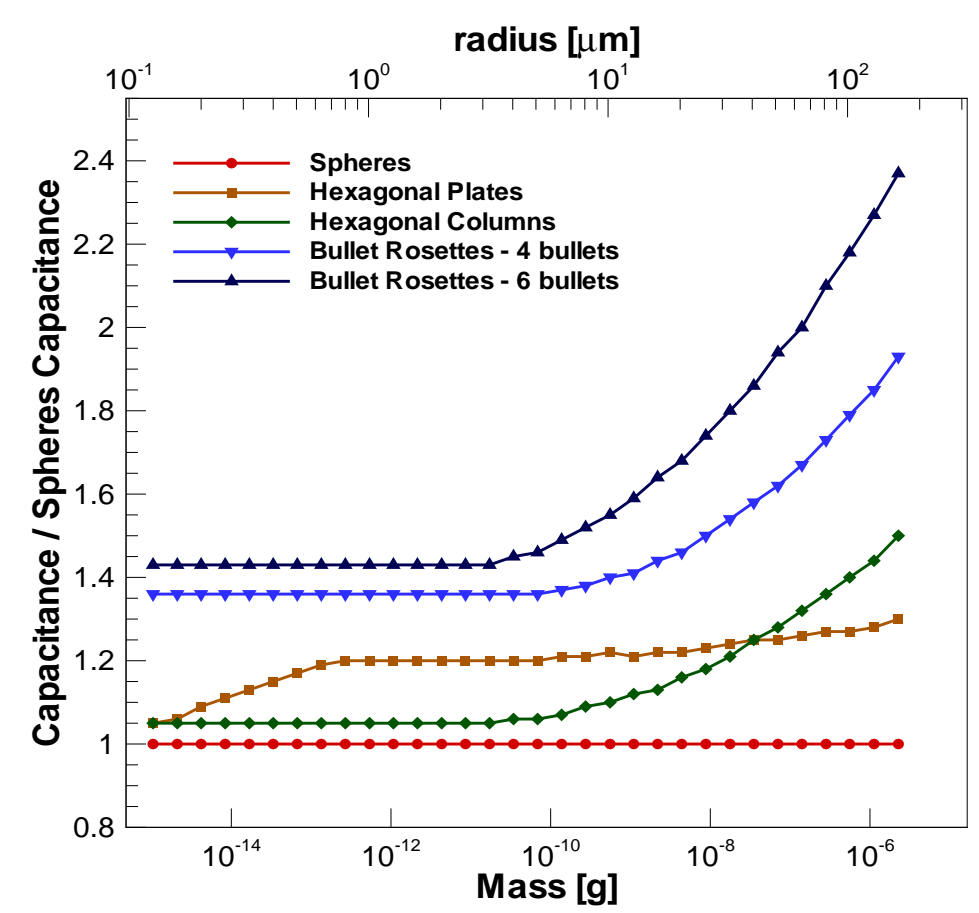

Figure 3.6: Capacitance of spheres, hexagonal columns, plates, and bullet rosettes with 4 and 6 bullets normalized respect to spheres Westbrook et al. (2008)

A comparison between the capacitance of spheres, hexagonal columns, plates, and bullet rosettes ice crystals at the same mass is shown in figure 3.6. The figure shows the capacitance has the same behavior that was found by Liu et al. (2003). The bullet rosettes ( 6 or 4 bullets) have the largest capacitance, the medium values correspond to hexagonal columns, and finally the smallest value is for spheres. This behavior in the capacitance is important in the contrail evolution at late time where the ice crystals can grow large enough to precipitate.

\subsubsection{Ventilation Coefficient $f_{v}$}

The ice crystals in the contrail evolution are moving with respect to water vapor in the ambient air. The vapor is distributed around the ice particles and the diffusional growth rate of ice crystals is influenced by the motion. Pruppacher and Klett (1997) 
had defined the effect that drives the mechanism of growth or sublimation as the ventilation effect. In the contrail evolution at late time it is very important when particles are large enough to have appreciable fall velocities.

The ventilation coefficient is a function of $S c=\frac{\mu}{\rho D}$ (Schmidt number) and $R e=$ $\frac{\rho V_{\text {fall }} D_{c}}{\mu}$ (particle Reynolds number). The characteristic maximum dimension $D_{c}$ of the ice particle involves the aspect ratio, which is calculated first. This dimension for the case of spheres is the diameter $(2 r)$, for the hexagonal columns is the basal face width $(w)$, and for bullet rosettes is the maximum dimension $\left(D_{\max }\right)$.

1. Spheres: CARMA version 2.3 in the microphysics model includes an equation for the ventilation coefficient for spherical ice crystals that was taken from Hall and Pruppacher (1976):

$$
f_{v}= \begin{cases}1.0+0.14 X^{2} & X<1.0 \\ 0.86+0.28 X & X \geq 1.0\end{cases}
$$

where: $X=S c^{1 / 3} R e^{1 / 2}$

2. Hexagonal Columns: The ventilation coefficient is represented by a secondorder polynomial described by Johnson (1997) using the results of Ji and Wang (1999).

$$
f_{v}=1.0+0.0309 X+0.1447 X^{2}
$$

3. Hexagonal Plates: The ventilation coefficient is defined as hexagonal columns.

$$
f_{v}=1.0+0.0105 X+0.0228 X^{2}
$$


4. Bullet Rosettes 4 and 6 - bullets: The ventilation coefficient is based on

$$
f_{v}=1.0+0.30059 X-0.0022 X^{2}
$$

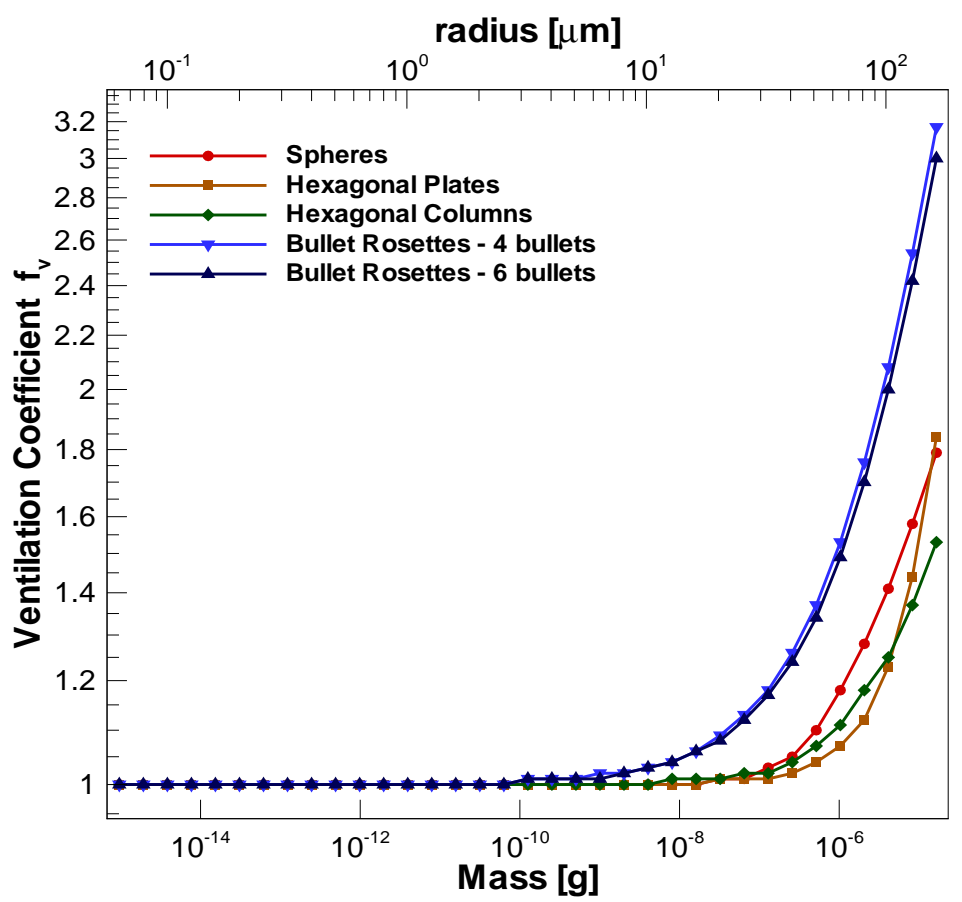

Figure 3.7: Ventilation coefficients for spheres, hexagonal columns, plates, and bullet rosettes

Figure 3.7 shows ventilation coefficients versus ice mass (or radius). The results show that the ventilation coefficient varies significantly with ice crystal shape, where the value of $X$ is a function of the aspect ratio through the approximation of the Reynolds number $R e$ for non-spherical shapes (specifically for the hexagonal column and bullet rosette shapes). Bullet rosettes have a higher $f_{v}$ value compared with spheres and hexagonal columns at the same ice mass. This is likely due to their higher surface area. 


\subsubsection{Fall Velocity $V_{\text {fall }}$}

\section{Spherical Ice Shape}

The contrail ice crystal terminal velocities are of primary importance in determining the rate of precipitation from upper- to middle-tropospheric levels and profoundly influence the precipitation at the largest supersaturation levels and later times. An accurate relationship between fall velocity and shape of a single ice crystal is needed to carry out a realistic contrail simulation.

The binned ice microphysics included in CARMA version 2.3 has a particular treatment of the fall velocity for spherical ice crystals, assuming that the small particles fall in the Stokes-Cunningham regime $R e<0.01$. The first step is to calculate the Stokes fall velocity, using the following expression:

$$
V_{\text {fall }}=\frac{2 r^{2} g\left(\rho_{i}-\rho\right)}{9 \mu f_{1}} S_{f}, \quad S_{f}=1+f_{2} K_{n}\left(c_{1}+c_{2} e^{-c_{3} / K_{n}}\right)
$$

The Cunningham correction for slip-flow, $S_{f}$, accounts for gas kinetic effects near the particle surface, with parameters $c_{1}=1.246, c_{2}=0.42$, and $c_{3}=0.87$. The shape correction factors for spheres are $f_{1}=1$ and $f_{2}=1$ (Toon et al., 1989).

Using the velocity obtained, we calculate the Reynolds number, with the simple equation:

$$
R e=\frac{2 \rho r V_{\text {fall }}}{\mu}
$$


For the regime $R e<1000$,

$$
V_{\text {fall }}=\frac{R e \mu}{2 r \rho}
$$

And finally for $R e>1000$ the drag coefficient is $C d=0.45$ independent of Reynolds number. The fall velocity is reached when the weight of the object is exactly balanced by the upward buoyancy force and drag force. That is:

$$
W=F_{b}+D_{g}
$$

If the falling object is spherical in shape, the expressions for the three forces are given below:

$$
\begin{aligned}
& W=\frac{4}{3} \pi r^{3} \rho_{i} g \\
& F_{b}=\frac{4}{3} \pi r^{3} \rho g
\end{aligned}
$$

The value of $F_{b}$ can be considered negligible.

$$
D_{g}=\frac{1}{2} C d \rho V^{2} A, \quad A=\pi r^{2}
$$

Substitution of equations ( 3.26 - 3.28) in equation 3.25 and solving for terminal velocity, $V_{\text {fall }}$ to yield the following expression:

$$
V_{f a l l}=S_{f} \sqrt{\frac{8 r g \rho_{i}}{3 C d \rho}}
$$




\section{Hexagonal Column Ice Shape}

For the hexagonal column shape, the binned ice microphysics model in CARMA version 2.3 does not have implemented the fall velocity. In order to use the hexagonal column shape it was necessary to find an adequate approximation for the fall velocity. The approach chosen was that used in Spichtinger and Gierens (2008) and Unterstrasser et al. (2008), only for ice particles ( $L \leq 1899 \mu \mathrm{m})$. The fall velocity is based in mass-length relations from Heymsfield and Iaquinta (2000b). The fall velocity as a function of ice crystal mass is:

Mass-length relations

$$
V_{o}\left(m_{i}\right)=\gamma\left(m_{i}\right) m_{i}^{\delta\left(m_{i}\right)}
$$

The values of piecewise constant parameter functions $\gamma\left(m_{i}\right)$ and $\delta\left(m_{i}\right)$ are given

in table 3.2. The transition masses are $m_{1}=2.146 \times 10^{-13} \mathrm{~kg}, m_{2}=2.166 \times 10^{-9}$ $\mathrm{kg}$, and $m_{3}=4.264 \times 10^{-8} \mathrm{~kg}$.

Table 3.2: Piecewise constant parameter functions for fall velocity

\begin{tabular}{ccc}
\hline \multicolumn{2}{c}{ Values for $\gamma, \delta$ in the velocity-mass } \\
\hline$m_{i}$ & $\gamma\left(m_{i}\right)$ & $\delta\left(m_{i}\right)$ \\
\hline$m_{i} \leq m_{1}$ & 735.4 & 0.42 \\
\hline$m_{1} \leq m_{i} \leq m_{2}$ & 63292.4 & 0.57 \\
\hline$m_{2} \leq m_{i} \leq m_{3}$ & 329.8 & 0.31 \\
\hline$m_{3} \leq m_{i}$ & 8.85 & 0.096 \\
\hline
\end{tabular}

The terminal velocities $V_{o}\left(m_{i}\right)$ are valid for reference values of $T_{0}=233 \mathrm{~K}$ and $P_{0}=300 \mathrm{hPa}$; for other temperatures and pressures we need to apply the correction factor Spichtinger and Gierens (2008):

$$
C f(T, p)=\left(\frac{P}{P_{0}}\right)^{(-0.178)}\left(\frac{T}{T_{0}}\right)^{(-0.394)}
$$


such that the terminal velocity is:

$$
V_{\text {fall }}\left(m_{i}, T, p\right)=\gamma\left(m_{i}\right) m_{i}^{\delta\left(m_{i}\right)} C f(T, p)
$$

\section{Hexagonal Plate and Bullet Rosette Ice Shapes}

Westbrook (2007) suggests a modified Stokes solution for a sphere as the fall velocity equation (equation 3.34) for non-spherical ice particles. The effective

radius replaces the radius of the sphere, but the problem is to find the effective radius for non-spherical particles.

Westbrook (2007) found that the capacitance is the appropriate length scale that characterizes the crystal viscous drag " $r "=$ " $C$ ". In our simulations the fall velocity of hexagonal plate and bullet rosette ice crystals is approximated by the equations 3.33 and 3.34 and using the capacitance calculated previously in equations $3.15,3.16$ and 3.17 .

The expression of fall velocity for horizontally oriented planar and bullet rosette crystals are respectively as follow :

$$
\begin{aligned}
& V_{\text {fall }}=\left(\frac{g}{8 \pi \mu}\right) \frac{m_{i}}{C} \\
& V_{\text {fall }}=\left(\frac{g}{6 \pi \mu}\right) \frac{m_{i}}{C}
\end{aligned}
$$

A comparison between the fall velocities of spheres, hexagonal columns, plates, and bullet rosettes is shown in figure 3.8. As expected the bullet rosette ice crystals have lower fall velocities than spherical and hexagonal column ice particles of the same mass. The difference is clearly observed in almost all of the 


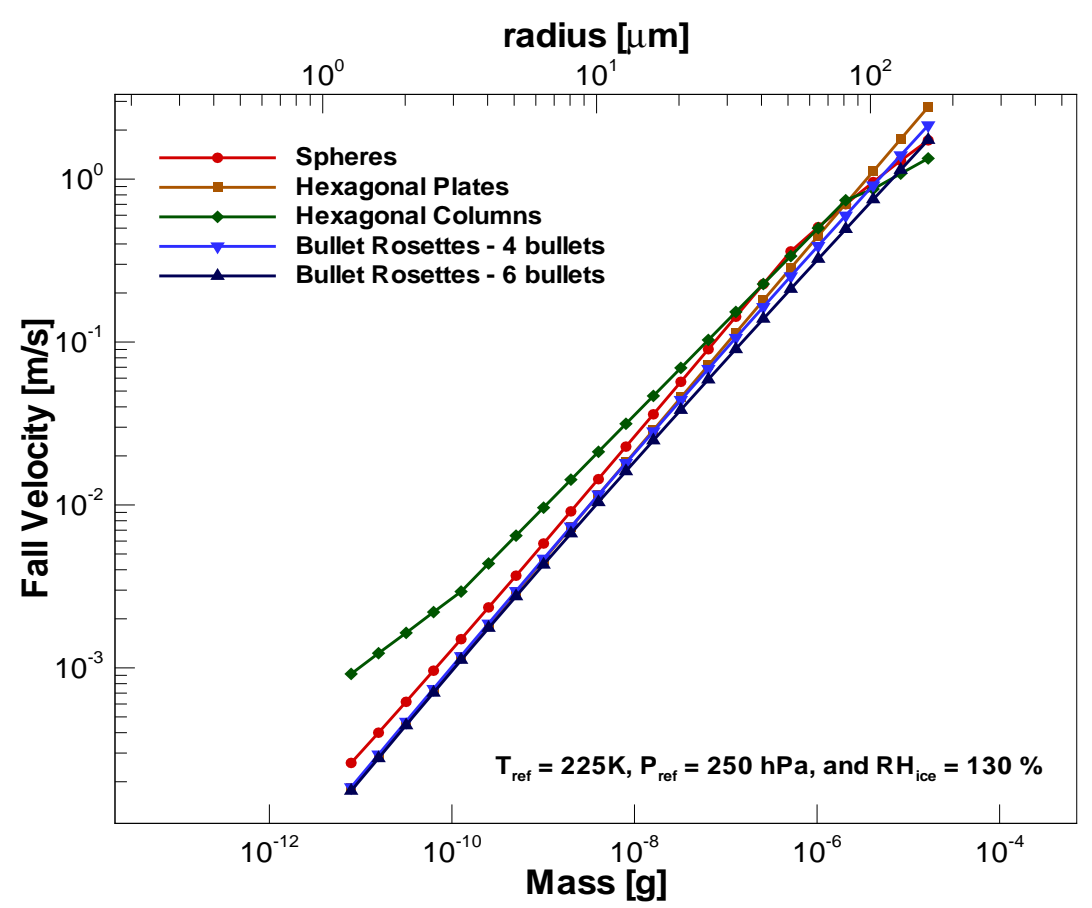

Figure 3.8: Fall velocities for spheres, hexagonal columns, plates, and bullet rosettes

spectrum, except for the larger ice particles. The contradictory velocity difference in larger ice particles is due to inconsistent handling; for some no high Reynolds correction have been included, for other (e.g., the hexagonal columns) empirical data was used that is not specific to that shape. In order to analyze the influence of larger ice crystals in contrail simulations, the case with large crystals was selected for this study. In older contrails with large crystals, the fall velocities increase larger than the mixing velocities and precipitation reaches an important level, resulting in a rapid increase of the contrail vertical extent. During this precipitation process at around 1.5 hours of simulation, the average logarithm spectral ice bin distribution $d M / d \log (r)$ reaches a pick at almost 100 $\mu \mathrm{m}$ (figure 3.9), where the fall velocities shows inconsistencies. The large particles in the spectral distribution for older contrail shows an important influence 
in the final results. A better approximation will be needed in the future to have in a realistic manner the dynamical evolution of older contrails.

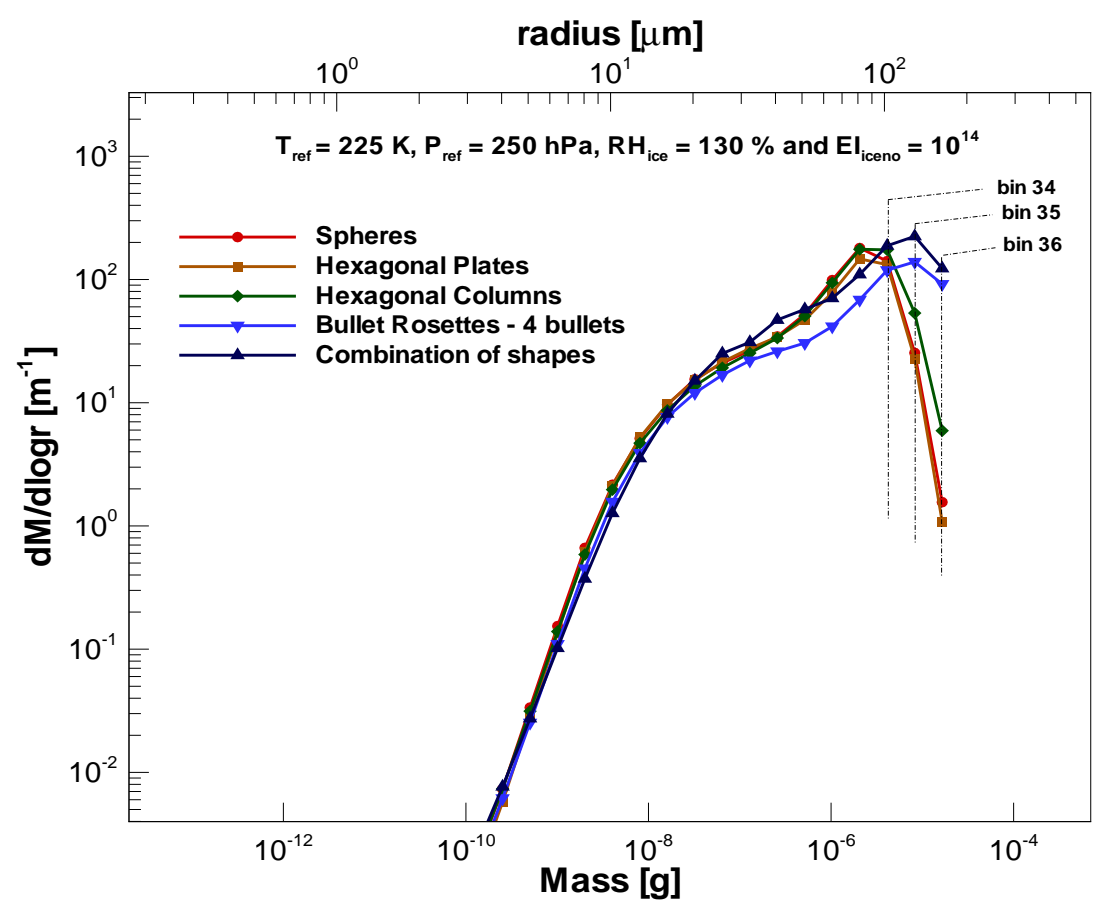

Figure 3.9: Average logarithm spectral ice bin distribution of the contrail plume at late time

\subsubsection{Radiation Effects on Contrails}

The WVU contrail code includes calculation of radiative heating and cooling taken from CARMA v2.3. The radiation parameters are calculated assuming Mie scattering for spheres. The spectral radiation covers the shortwave spectrum (Solar - SW) with 26 wavelength bins $(0-4.292 \mu \mathrm{m})$ and longwave (infrared - LW) with 18 bins (4.546-62.50 $\mu \mathrm{m})$.

The radiation computation included 10 vertical layers between the surface and the bottom of the LES domain. The calculation of the downwelling infrared 
radiation at the domain top assumed a temperature of $130 \mathrm{~K}$. The water vapor column above the domain is not considered on the simulation. The cosine of the solar zenith angle was held fixed at 0.5. In older contrails, ice particles fall in random orientation; an initial study of the shape effect on simulations coupled with radiation included the shape-dependent crystal cross-sectional areas. In order to simplify the calculation of their cross-sectional areas the following simple orientations were assumed: sphere (default), hexagonal column (horizontally), hexagonal plates (vertically) and bullet rosette (number of bullets $\times$ hexagonal columns - horizontally). As a consequence the cross-sectional areas were expressed as follows:

- Sphere:

$$
A_{c}=\pi r^{2}
$$

- Hexagonal column:

$$
A_{c}=2 s L
$$

- Hexagonal plate:

$$
A_{c}=1.5 \sqrt{3} s^{2}
$$

- Bullet rosette:

$$
A_{c}=2 n_{b} s L
$$

\subsubsection{Ice Deposition Coefficient $\beta_{i}$}

As part of this study we also have analyzed the influence of the ice deposition coefficient (or mass accommodation coefficient) on the ice contrail metrics. The mass accommodation or sticking coefficient of water vapor over a solid ice surface 
has been considered by many researchers experimentally and theoretically giving conflicting results for $\beta_{i}$. Table 5.5 from Pruppacher and Klett (1997) presents results ranging over more than two orders of magnitude $(0.014-1.0) . \quad \beta_{i}$ is defined as the ratio of number of atoms (or molecules) that adsorb, or "stick", to a surface to the total number of atoms that impinge upon that surface during the same period of time. Its value is between 1.00 (all impinging atoms stick) and 0.00 (none of the atoms stick).

The value of $\beta_{i}$ (CARMA default value 0.93 ) is included in the WVU contrail code through kinetic theory modifications to the diffusion coefficient D for small ice crystals. In CARMA the effective diffusion coefficient is given by:

$$
D^{*}=D /\left(1+\lambda k_{n}\right) \quad \text { with } \quad \lambda=\left(\frac{4}{3} k_{n}+.71\right) /\left(k_{n}+1\right)+\frac{4}{3} \frac{\left(1-\beta_{i}\right)}{\beta_{i}}
$$

where the Knudsen number is $k_{n}$, the ratio of the molecular mean free path in the gas to the particle radius.

Modeling studies in cirrus clouds have analyzed the effects of $\beta_{i}$ on the homogeneous nucleation of new ice crystals. The ice deposition coefficient directly influences the growth rate of small ice particles and hence the ice crystal number density that results (Lin et al., 2002). The contrail simulations here do not include nucleation; the evolution of ice particles is only by diffusional growth. However, contrail simulation of young and older contrails involves mainly processes related to small ice crystals. The mass growth rate (equation 3.1) depends directly on the diffusion coefficient through equation 3.2. The dependence of $D^{*}$ on $\beta_{i}$ will be small if $k_{n}$ is small (large ice crystals) or if $\beta_{i}$ is close to 1 . (CARMA has as default value 0.93). The decrease in the diffusion coefficient (by 
decreasing $\beta_{i}$ ) reduces the impact of the Kelvin effect and so more ice particles are conserved in the plume. 


\section{Chapter 4}

\section{Contrail Simulation}

Detailed modeling of aircraft contrail requires an explanation of the initial setting to understand the simulation process.

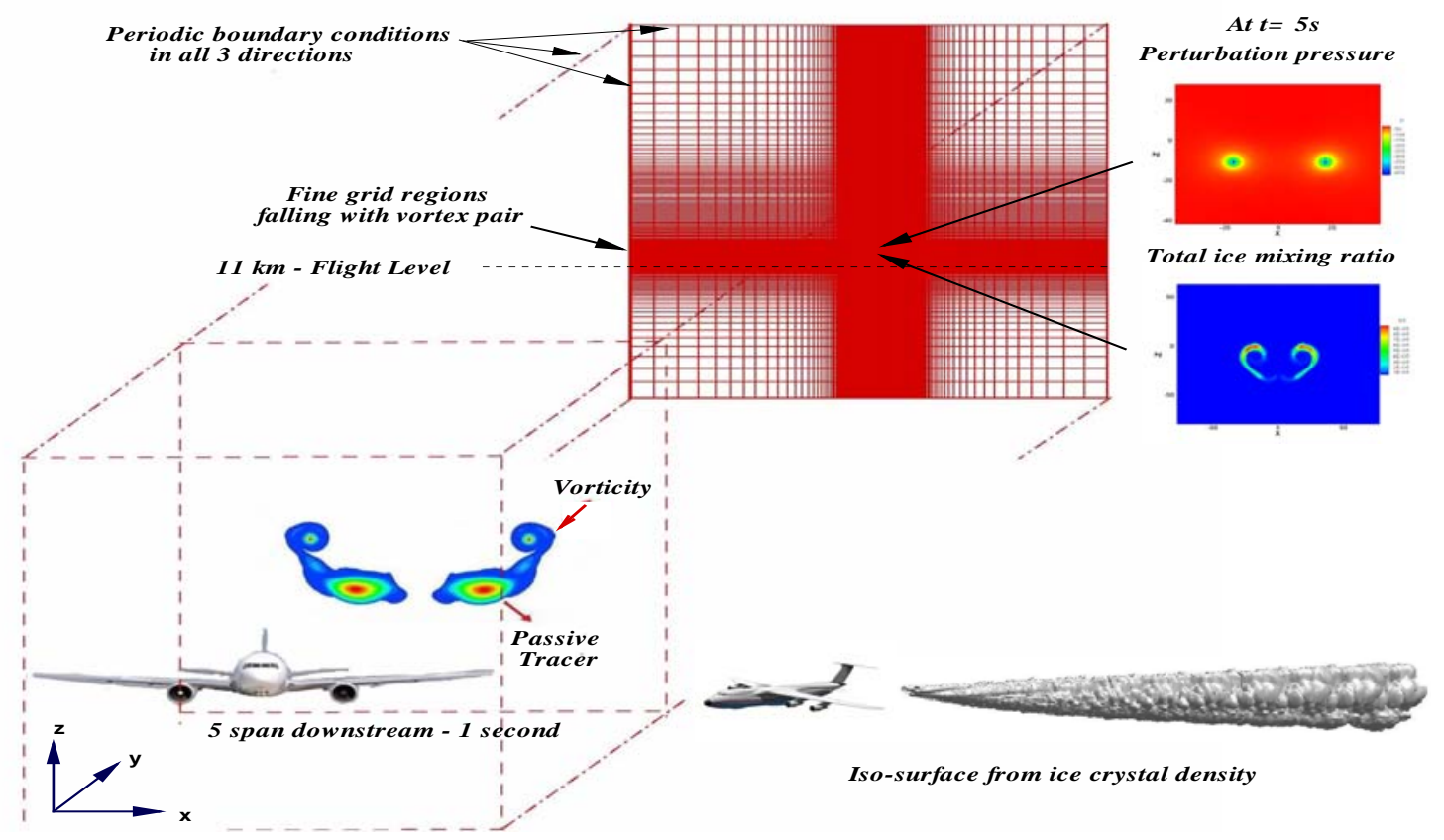

Figure 4.1: Simulation Setup - axes and typical grid configuration 
Firstly, the coordinate system is defined as " $\mathrm{x}$ ", " $\mathrm{y}$ ", and " $\mathrm{z}$ " in the cross-stream, down-stream, and vertical direction respectively (figure 4.1). Periodic boundary conditions were employed in all three directions. The stretched grid permits the boundaries to be moved far from the wake, minimizing the sensitivity to the boundary conditions (Lewellen and Lewellen, 1996). The aircraft and flight parameters used in the present simulations are defined in table 4.1.

Table 4.1: Boeing 767 parameters at the jet exit:

\begin{tabular}{lll}
\hline Type & Boeing 767 & {$[-]$} \\
\hline Altitude & 11 & $\mathrm{~km}$ \\
\hline Flight speed & 236.9 & $\mathrm{~m} / \mathrm{s}$ \\
\hline Vortex circulation & 390.7 & $\mathrm{~m}^{2} / \mathrm{s}$ \\
\hline Geometric wing span & 47.24 & $\mathrm{~m}$ \\
\hline Vortex pair spacing & 19.4 & $\mathrm{~m}$ \\
\hline Initial fall velocity of vortex system & 1.61 & $\mathrm{~m} / \mathrm{s}$ \\
\hline Fuel flow & 5.78 & $\mathrm{~kg} / \mathrm{km}$ \\
\hline Emission index of water & 1.25 & $\mathrm{~kg} / \mathrm{kg}$ \\
\hline Assumed engine efficiency & 30 & $\%$ \\
\hline
\end{tabular}

\subsection{Initial Conditions and Simulation Sequence}

The contrail simulation is initialized from the Boeing 2D-wake field taken at one second (or $\sim 5$ spans) downstream. The data contains information such as vorticity level, temperature, velocities and passive tracer concentration that are read and interpolated onto the contrail grid. The WVU contrail code has the capability to simulate contrails in fully three-dimensional (3D) and quasi-two dimensional (Q2D) modes. Fully-3D contrail simulation captures in detail the three-dimensional evolution of the wake, vortex pair interaction and decay. On 
the other hand, Q2D simulation only includes some of the important physics of the contrail evolution and will be explained in more detail in section 4.6.

The sequence of a basic 3D simulation has been widely explained in previous studies (Lewellen and Lewellen, 2001a).

\subsubsection{Young Contrails}

The "early time" simulation from 0-1000 s covers the evolution of the vortex system, linking process, ring formation and break up. During this period, more grid resolution is needed to capture the dynamical evolution of the vortex wake. In young contrails (less than half hour) the core contains a high concentration of small ice crystals with insignificant fall velocities. However, the edges of the plume are characterized by high supersaturation and hence the ice crystals become large enough to acquire an adequate fall velocity to precipitate. Before the precipitation begins, the vortex dynamics that drives the contrail evolution almost dies out. After this period, the grid resolution is relaxed, but the simulation domain is increased in size.

\subsubsection{Older Contrails}

Persistent older contrails (ages $\sim$ hours) are characterized by an increase in their volumes, dispersion by atmospheric turbulence and vertical extent by precipitation process. The change in the initial morphology of the plume (horizontal dispersion by wind shear and vertical extent by the precipitation process) re-

quires a larger domain. This second stage is called the "late time" simulation period (1000-30000 s). 


\subsubsection{Grids}

A complete simulation of young contrails in $3 \mathrm{D}$ often requires different grids for different stages: jet development, prelinking, vortex linking, late vortex and buoyant plume and 2 or more grids for older contrails. Q2D simulations often only need 1 grid each for young and older contrails. Depending on the initial configuration of the case in study a different domain will be needed. Table 4.2 summarizes all grids used in this study:

Table 4.2: Grids for Contrail Simulations

\begin{tabular}{|c|c|c|c|c|c|}
\hline & Case & Description & Type & Domain $(\mathrm{m}) \mathrm{XxYxZ}$ & Grid points \\
\hline $3 \mathrm{D}$ & Base Case & Jet Development & Young & $840 \times 52 \times 905$ & $90 \times 130 \times 136$ \\
\hline $3 \mathrm{D}$ & Base Case & Prelinking & Young & $840 \times 260 \times 1058$ & $76 \times 130 \times 128$ \\
\hline $3 \mathrm{D}$ & Base Case & Vortex Linking & Young & $840 \times 260 \times 1058$ & $62 \times 162 \times 121$ \\
\hline $3 \mathrm{D}$ & Base Case & Late Vortex & Young & $840 \times 260 \times 1042$ & $77 \times 130 \times 122$ \\
\hline $3 \mathrm{D}$ & Base Case & Buoyant Plume & $\begin{array}{l}\text { Young } \\
\end{array}$ & $1520 \times 520 \times 1656$ & $51 \times 126 \times 137$ \\
\hline $3 \mathrm{D}$ & Wind Shear & Jet Development & $\begin{array}{l}\text { Young } \\
\end{array}$ & $840 \times 52 \times 905$ & $90 \times 130 \times 136$ \\
\hline $3 \mathrm{D}$ & Wind Shear & Prelinking & Young & $930 \times 260 \times 1058$ & $88 \times 130 \times 128$ \\
\hline $3 \mathrm{D}$ & Wind Shear & Vortex Linking & Young & $1280 \times 260 \times 1058$ & $82 \times 162 \times 115$ \\
\hline $3 \mathrm{D}$ & Wind Shear & Late Vortex & $\begin{array}{l}\text { Young } \\
\end{array}$ & $1280 \times 260 \times 1042$ & $97 \times 130 \times 122$ \\
\hline $3 \mathrm{D}$ & Wind Shear & Buoyant Plume 1 & $\begin{array}{l}\text { Young } \\
\end{array}$ & $1960 \times 520 \times 1656$ & $88 \times 126 \times 137$ \\
\hline $3 \mathrm{D}$ & Wind Shear & Buoyant Plume 2 & $\begin{array}{l}\text { Young } \\
\end{array}$ & $2400 \times 520 \times 1656$ & $151 \times 66 \times 138$ \\
\hline Q2D & Base Case & Jet Development & Young & $400 \times 40 \times 993$ & $94 \times 8 \times 149$ \\
\hline Q2D & Base Case & Complete sequence & Young & $1520 \times 40 \times 1670$ & $97 \times 8 \times 168$ \\
\hline Q2D & Base Case & Complete sequence & Older & $12000 \times 240 \times 5040$ & $155 \times 8 \times 189$ \\
\hline Q2D & Wind Shear & Jet Development & Young & $400 \times 40 \times 778$ & $94 \times 8 \times 149$ \\
\hline Q2D & Wind Shear & Complete sequence & Young & $1520 \times 40 \times 1670$ & $97 \times 8 \times 168$ \\
\hline Q2D & Wind Shear & Sequence 1 & Older & $16000 \times 200 \times 4040$ & $160 \times 8 \times 177$ \\
\hline Q2D & Wind Shear & Sequence 2 & Older & $32000 \times 400 \times 4050$ & $160 \times 8 \times 117$ \\
\hline Q2D & Radiation & Complete sequence & Older & $12000 \times 240 \times 7000$ & $155 \times 8 \times 238$ \\
\hline
\end{tabular}




\subsection{Ambient Field}

A quantity dynamically important is the potential temperature $\theta$. It is conserved for all dry adiabatic processes. The potential temperature of a parcel at pressure $\mathrm{P}$ commonly defined as the temperature that the parcel would posses if adiabatically brought to a standard reference pressure $P_{o}$, usually 1000 millibars or $\mathrm{hPa}$. In our study the reference pressure is taken as that at flight level, so $\theta=T$ there.

The lapse rate used in almost all simulation was $2.5 \mathrm{~K} / \mathrm{km}$ and the level of $R H_{\text {ice }}$ was chosen depending of the case in study. These parameters where generated and placed in an ambient file that was read by the WVU contrail code during a simulation.

The mean ambient vertical profiles of potential temperature $\theta$, and $R H_{\text {ice }}$ used are given in figures 4.2 and 4.3 respectively.

\subsection{Ambient Turbulence and Wind Shear}

The ambient turbulence is created in a separate LES simulation, depending on the stage of contrail development. A minimal change in the initial velocity and temperature perturbation may produce completely different results. The ambient turbulence level is very important in contrail simulations, since it influences the mixing of ambient air with the contrail, among other effects.

The WVU contrail code includes the capability of automatically adding these turbulence fields in order to have a more realistic ambient in the simulation. A short summary about the turbulence generation model by Lewellen et al. (2009) and used in this study is presented here: 


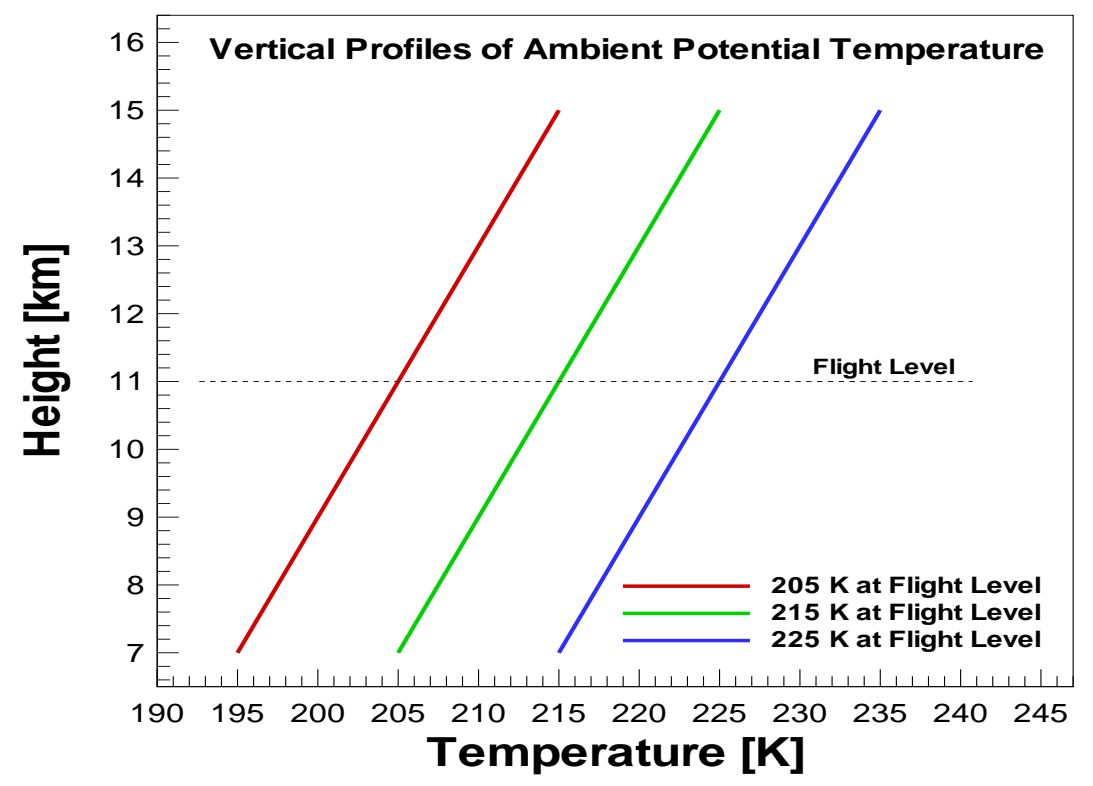

Figure 4.2: Vertical profiles of $\theta$ used in simulations

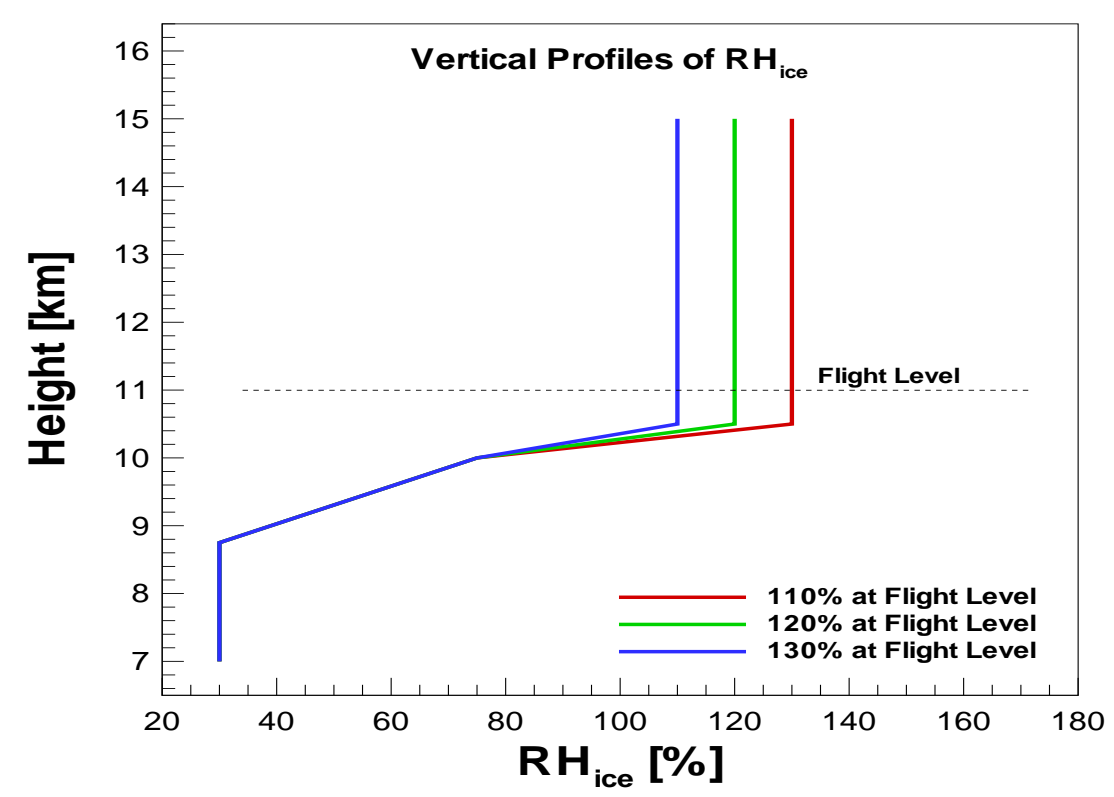

Figure 4.3: Vertical profiles of $R H_{i c e}$ used in simulations 
Contrail evolution is strongly affected by the level of turbulence. The generation of the turbulent ambient through a LES model is numerically expensive, due to the large domains and relatively fine grids used to capture in a realistic manner the atmospheric behavior. A quasi-steady turbulence field is enough to reproduce the turbulent behavior commonly found at flight level. The LES model is initialized with random temperature and velocity perturbation of a specific amplitude and superposed on the ambient stratification and mean shear. The simulation time depends on the desired level of decaying stratified turbulence, that generally occurs in several BruntVäisälä periods. A 3D velocity field perturbation and the subgrid turbulence kinetic energy are chosen at a specified age (decaying time), and added to the main LES simulation at specified times. The quasi-steady turbulence was set by parameters such as stratification, shear, amplitude of temperature perturbation, age of the field and periodical time of addition. A characteristic behavior in turbulence and gravity wave amplitudes is that they increase by increasing amplitude of temperature perturbation (and/or decreasing the periodical time of addition). The relative importance of gravity waves respect to turbulence is increased by increasing the age of the field (and/or periodical time of addition); and the level of intermittency increases as periodical time of addition is increased.

The ambient turbulence in the contrail evolution was developed for two well defined stages: young and older contrails. In young contrails, the ambient turbulence was treated following the approach used by (Lewellen and Lewellen, 1996; Lewellen et al., 1998). The perturbations in the ambient turbulence generate the initial growth of the Crow instability observed in the evolution of aircraft trailing vortices. The addition of turbulence into the contrail simulation is based on the initial configuration of the case in study. The optimal 
field is that after the fluctuations have settled down. The turbulence ambient field selected needs to have both long and short wavelength disturbances. Long wavelength disturbance is needed to activate the Crow instability and the some small wavelength to provide the appropriate mixing. In a field selected too early, the turbulent fluctuations will be too high, which is an unrealistic ambient turbulence field for normal aircraft operation. On the other hands, in a field much later, the small scale features are gone. In early contrails, the first ambient turbulence (age $\mathrm{t}=1300 \mathrm{~s}$, see figure 4.4) is generated in a separate LES run and added to the main 3D simulation.

Previous studies established that different levels of turbulence could modify the timescale of vortex linking, among other things. Lewellen and Lewellen (2001a) showed that at reference values of $T_{\text {ref }}=220 \mathrm{~K}$ and $P_{\text {ref }}=250 \mathrm{hPa}$, the linking process occurs around 90-100 s . However, for the highest level of atmospheric turbulence the initial vortex linking process could occur at $\sim 50$ s. The turbulence dissipation rate was parameterized by $\epsilon_{t}=b_{t} q^{3} / \Lambda$, where $b_{t}=$ $1 / 8$ is a constant, $1 / 2 q^{2}=$ subgrid turbulent kinetic energy per mass and $\Lambda=$ subgrid length scale, with the corresponding values of $\epsilon_{t}=5.6 \times 10^{-6} \mathrm{~m}^{2} \mathrm{~s}^{-3}$ and $\epsilon_{t}=5.8 \times 10^{-5} \mathrm{~m}^{2} \mathrm{~s}^{-3}$ respectively.

In the simulation of older contrails, the generation of a realistic ambient turbulence is complicated. At altitudes, where aged aircraft contrails evolve, the characterization, generation and maintenance of the atmospheric ambient is complex, since it is produced by a combination of atmospheric turbulence, gravity waves, wind shear and atmospheric stratification. Older contrail dispersion is mainly driven by atmospheric turbulence, waves, crystal precipitation and buoyant forcing by radiation. After the vortex pairs have almost die the atmospheric ambient needs to be re-energized from time to time to mimic a real 

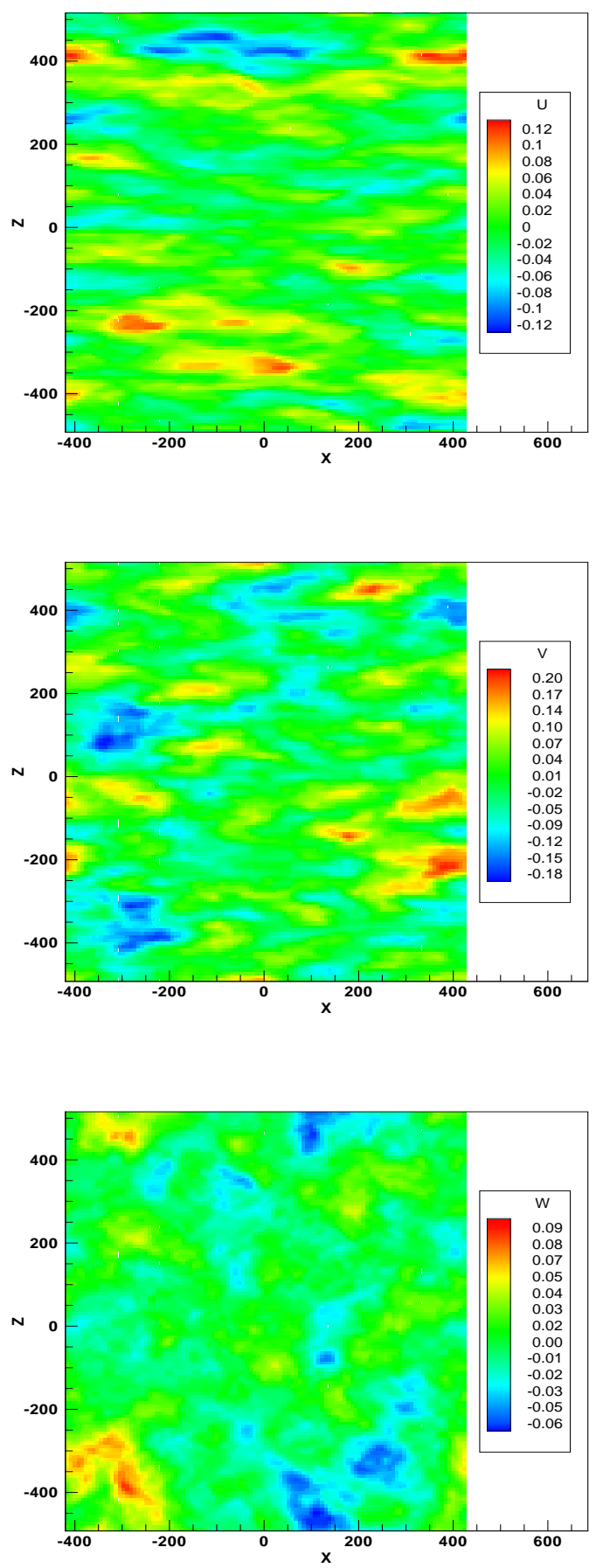

Figure 4.4: Ambient Turbulence $(\mathrm{m} / \mathrm{s})$ from $0-1300 \mathrm{~s}$, for the case at $T_{r e f}=225 \mathrm{~K}$, cross-stream slice. 
atmosphere.

In older contrails, to have a realistic atmosphere implies a periodic addition of turbulence. The period addition of atmospheric turbulence can generate a nonrealistic accumulative process of added velocity fields perturbation. In order to avoid this and have independent periodic turbulence addition, the horizontal mean average velocities are subtracted off and the entire domain is shifted a random amount in the $\mathrm{x}, \mathrm{y}$ and $\mathrm{z}$ direction. This process is realized in the simulation prior each periodic turbulence addition. The temperature perturbation is not added into the simulation since it can affect the ice microphysics dynamics of the contrail. For the case of older contrails with large ice crystals, from the separate LES run an aged turbulence field $t=21600 \mathrm{~s}$, figure 4.5 is added periodically to the main simulation.

The level of atmospheric turbulence is directly related to the combination of parameters that determine the quasi-steady turbulence. Based in previous studies of D.C. Lewellen, two levels of turbulence were used in this study: high turbulence level has a temperature perturbation of 2 , with $600 \mathrm{~s}$ at age of the field and periodical time addition of 600s; while low turbulence level has a temperature perturbation of $2 \times 10^{-1}$, with $3600 \mathrm{~s}$ at age of the field and periodical time addition of 3600s. The turbulence level was roughly quantified, as in Lewellen et al. (2009), by the averaged values of small-scale dissipation rate and the vertical velocity variance. In cases where the statistics are highly intermittent, a range of values would be more accurate. For the case of "high" turbulence, the range values were $\left[1.8 \times 10^{-5}-1.8 \times 10^{-4}\right] \mathrm{m}^{2} / \mathrm{s}^{3}$ and $\left[2.5 \times 10^{-2}-7.1 \times 10^{-2}\right] \mathrm{m}^{2} / \mathrm{s}^{2}$, and in the case of "low" turbulence level $\left[1.1 \times 10^{-11}-8.2 \times 10^{-7}\right] \mathrm{m}^{2} / \mathrm{s}^{3}$ and $\left[6.5 \times 10^{-3}-1.7 \times 10^{-2}\right] \mathrm{m}^{2} / \mathrm{s}^{2}$ respectively.

The WVU contrail code also has the capability to simulate both a down-stream 

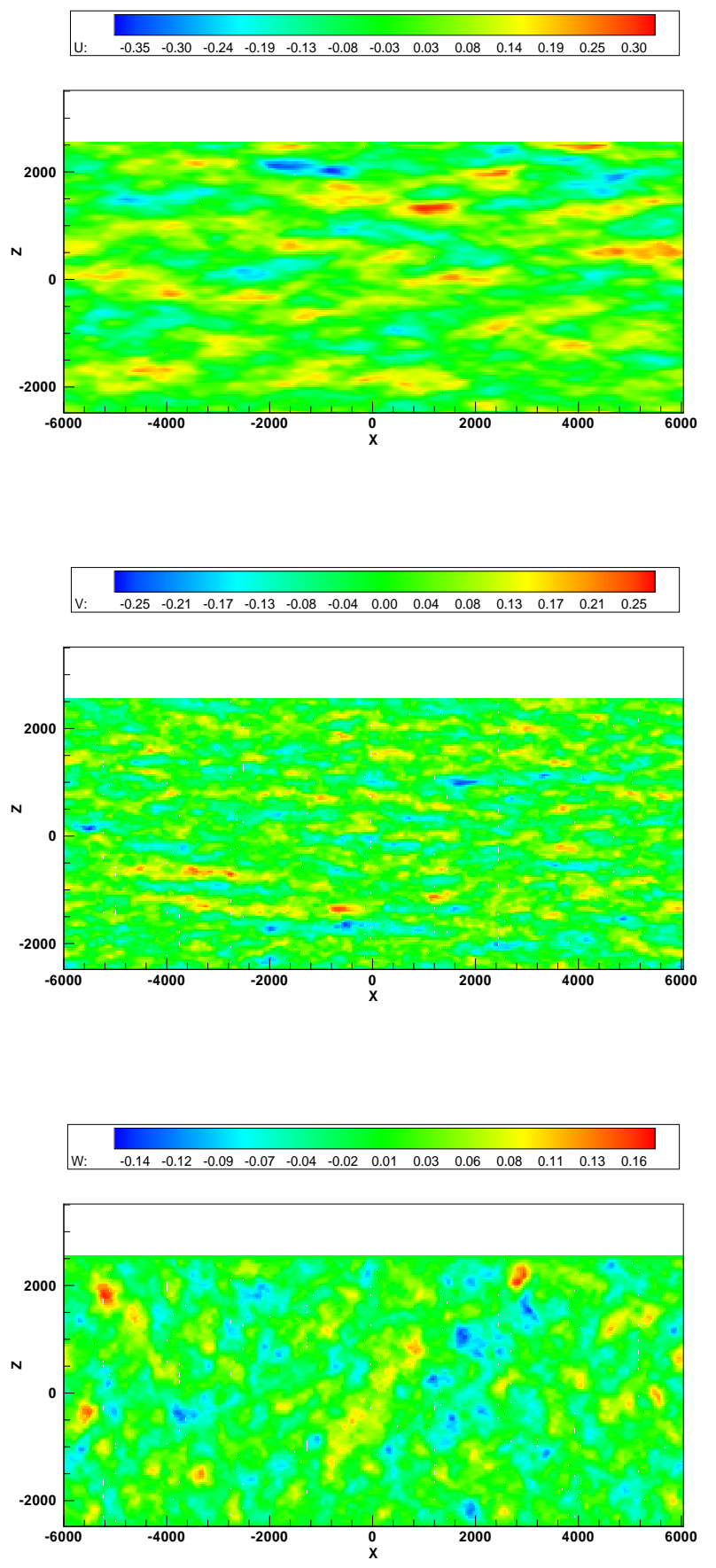

Figure 4.5: Ambient Turbulence $(\mathrm{m} / \mathrm{s})$ from $0-21600 \mathrm{~s}$, for the case at $T_{\text {ref }}=225 \mathrm{~K}$, cross-stream slice. 
shear and a cross-stream shear. The cross-stream wind shear is a linear vertical wind shear $\left(s^{-1}\right)$ that is perpendicular to the wake vortex axis $(d u / d z)$, while the down-stream is a vertical shear that is parallel to the flight path $(d v / d z)$. Typically, a contrail simulation with down-stream wind shear can be performed using the same grids as non-shear cases. However, wind shear in the crossstream direction can affect both the vortex dynamics and the ice development at early and late time. The dispersion and the smearing observed in the plume suggest using new grids expanding the domain in $\mathrm{x}$ direction to capture the new contrail characteristics.

\subsection{Description of the Simulation Cases}

Atmospheric conditions and aircraft parameters have been tested previously to establish the cases in this study. All cases involve ice supersaturated atmospheric ambients $\left(R H_{i c e} \geq 100 \%\right)$. $R H_{i c e}$ is defined as the ratio of the vapor pressure to the saturation vapor pressure with respect to ice. The reference temperature $T_{\text {ref }}$ and reference pressure $P_{\text {ref }}$ are defined at flight level (table 4.1). The number of ice crystals produced by the mass of fuel consumed, $E I_{\text {iceno }}$ (emission index of ice), defines the initial ice spectrum magnitude. The $E I_{\text {iceno }}$ values for the cases on study were chosen in order to have two well defined size ranges $\left(10^{14}\right.$ relative large and $10^{16}$ small crystals). The denomination of relative large and small crystals is based on previous studies. Low level of $E I_{\text {iceno }}$ and high $T_{\text {ref }}$ leads with large crystals, while high level of $E I_{\text {iceno }}$ and

low $T_{\text {ref }}$ wih small ice crystals. The used values are in agreement with previous works by Lewellen and Lewellen (2001a); Sussmann and Gierens (1999), and Gierens and Jensen (1998). The wind shear values in the cross-stream direction 
were chosen in modest mean levels. The binned microphysics was defined as a spectrum of 36 global and 18 local bins with a mass ratio between succesive bins of 2 .

The level of turbulence influences the mixing rate and the precipitation level. The cases in study the values were chosen as follows: base case I was simulated with "high" ambient turbulence level and added in the fields every 10 minutes (to study contrails formed by large ice crystals and the influence of precipitation process); and the base case II, with "low" ambient turbulence level and added every hour (to study contrail formed by small ice crystals and absence of precipitation).

The principal simulation set for the shape effect study is given in table 4.3 


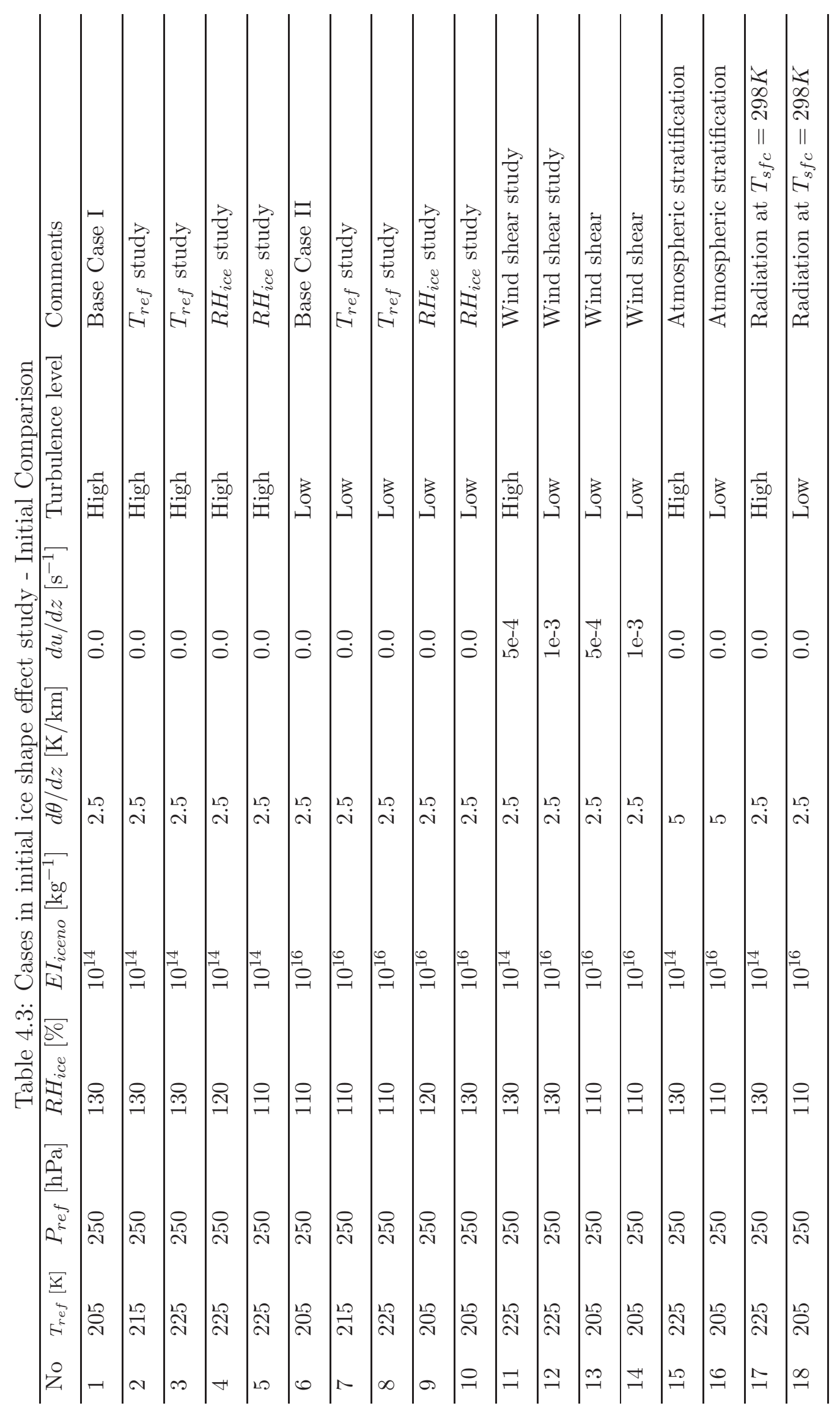


The reference temperatures were chosen based on the temperature statistics of measured values by Boeing $(205 \mathrm{~K}, 215 \mathrm{~K}$, and $225 \mathrm{~K})$. The level of supersaturation was selected in order to analyze the influence of available atmospheric moisture on the shape effect $(110 \%, 120 \%$, and 130\%). The atmosphere is normally turbulent, mainly by wind shear. The modest levels of mean wind shear chosen were $5 \times 10^{-4} \mathrm{~s}^{-1}$ and $1 \times 10^{-3} \mathrm{~s}^{-1}$.

The levels of atmospheric stratification were $2.5 \mathrm{~K} / \mathrm{km}$ and $5 \mathrm{~K} / \mathrm{km}$. The level $2.5 \mathrm{~K} / \mathrm{km}$ was used as default value for all simulations. The initial radiation study on contrails was focused on the base cases I and II and on the effect of the ice crystal size (effective radius $r_{e}$ ) on the radiative effects on contrails. The Earth's surface temperature value was $T_{s f c}=298 \mathrm{~K}$.

As an initial comparison we have defined two base simulations. We used the opposite ends of the final spectrum to define the cases: Base Case I mainly formed by large ice crystals due to the low level of $E I_{i c e n o}$, and by very small ice crystals for the Base Case II (see table 4.3).

\subsection{Three-Dimensional Simulation}

A basic review of the fluid dynamics and microphysical processes in contrail evolution is discussed here using the base case I and the Lewellen and Lewellen (2001a) previous reported results. In order to visualize the influence of the shape effect in the contrail fluid mechanics, we have used fully-3D simulations. The results will permit us to make a qualitative comparison of contrail evolution using different ice crystal shapes. Fully-3D contrail simulation is the best option, because it captures in a realistic manner the 3D wake dynamics present in the contrail evolution: the strong downward motion of the wake vortices and 
the expansion of the contrail plume through the mixing with the atmospheric ambient.

The analysis of the contrail fluid dynamics was made through the "drift plots". The drift plots were inspired by the scanning Lidar measurements of wakes in Lewellen et al. (1998). This type of plot shows the vertical distribution of the ice crystal number in the plume, as well as the evolution of the trailing vortices (touching process, vortex ring formation and the break up) with the help of the variable perturbation pressure. In the cross-stream direction, this plot shows the evolution of ice crystal number and ice mass. A drift velocity was defined as if the contrail were passing over a Lidar measurement chosen as $12 \mathrm{~m} / \mathrm{s}$ here.

\subsubsection{Young Contrail Dynamics}

As was shown in Lewellen and Lewellen (1996), the evolution of young contrails after initial formation is mainly driven by the wake dynamics. Figures 4.6 and 4.9 show that the vortex pair falls rapidly. As a consequence of atmospheric turbulence two velocity fields affect their evolution. The velocity field by the perturbation in the vortices that increase in a sinusoidal "mutual inductance" instability (The Crow instability) and the velocity field induced by each vortex itself "self induction". Both fields together permit the rapid descent of the vortex system with an initial velocity defined as $V f_{\text {init }}=\Gamma / 2 \pi b_{o}$.

Initially, the contrail plume falls down (Figure 4.6) and the competition between the compressional adiabatic heating process of the descending parts and the mixing with ambient fresh air drives an eventual ice crystal loss. The ice crystal diffusional growth is affected by the Kelvin effect directly related to the ice crystal habit (shape and size). The Kelvin effect plays an important role in the 


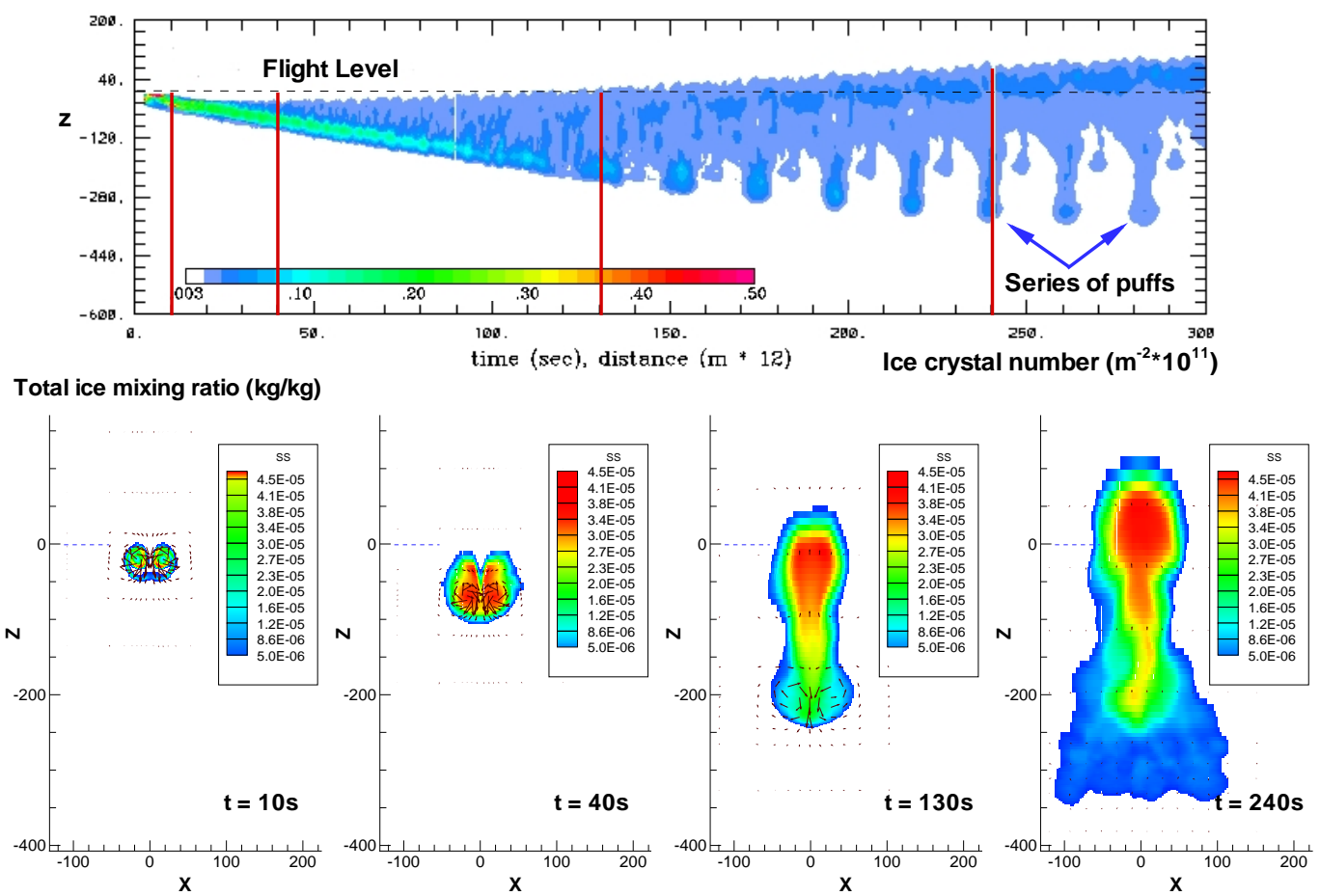

Figure 4.6: Contrail Simulation, 3D - Young Contrail - Total Mixing Ratio - CrossStream - Integrated Drift Plot (top) and Down-stream averaged contours (bottom)

ice physics. Its influence affects mainly the smaller ice crystals, which are lost by evaporation at a greater rate than large ones. This mechanism is clearly observed in young contrails, which are characterized by a high concentration of small ice particles and commonly observed at flight level.

Figure 4.7 (left) illustrates the influence of adiabatic heating and the Kelvin effect on the evolution of the number of ice crystals (decreasing in time by evaporation of the smaller ones). The reduction in concentration and the growth in size of the large ice crystals is observed in the shifting of the ice spectrum (right). Additionally, it is shown the evolution of the number of ice crystal when the Kelvin effect is turn off. The non-inclusion of this effect result in more number of ice crystal conserved in the plume (left). 

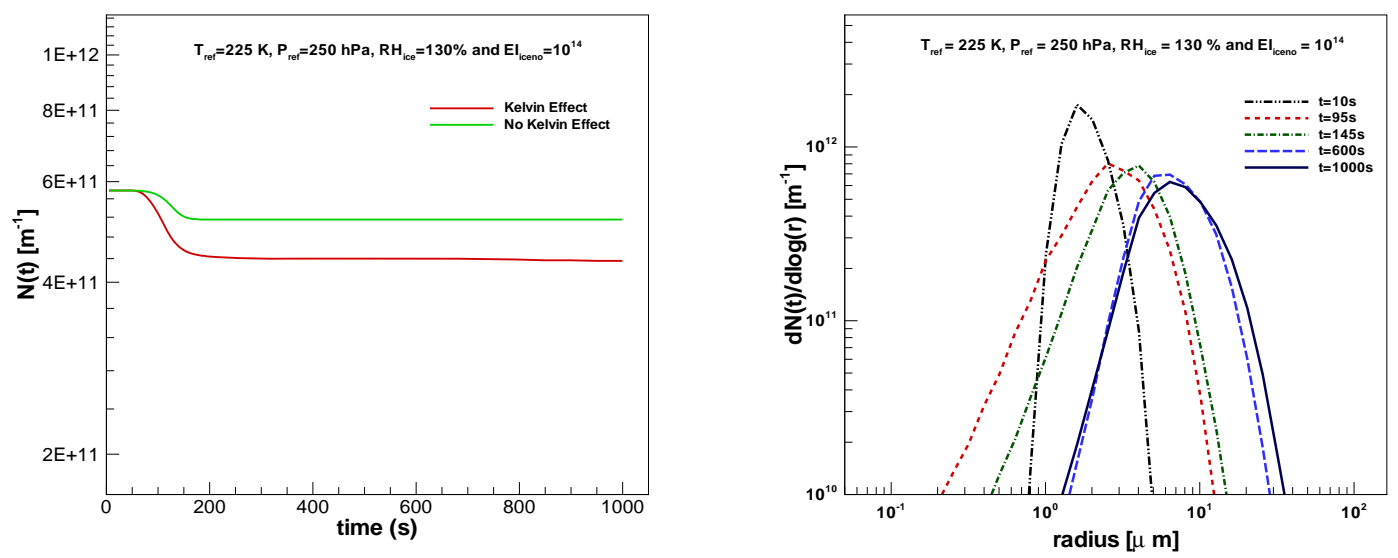

Figure 4.7: Total ice crystal number per meter of flight path, N(t) (left) and Contrail - mean ice spectrum at selected times (right)
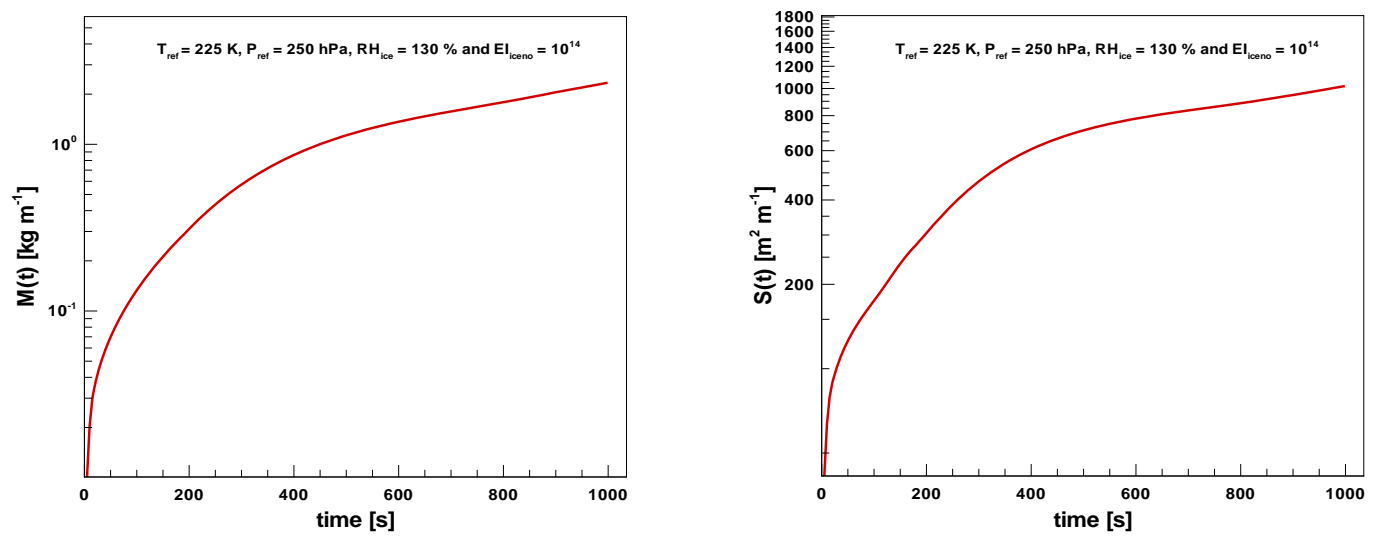

Figure 4.8: Ice mass $\mathrm{M}(\mathrm{t})$ (left) and Ice crystal surface area $\mathrm{S}(\mathrm{t})$ (right), both per meter of flight path

The surviving ice crystals of the period dominated by the adiabatic heating grow quickly during the first 300 s. The growth of larger ice crystals by the available moisture in young contrails is observed in ice mass $\mathrm{M}(\mathrm{t})$ and the ice surface area $\mathrm{S}(\mathrm{t})$ (figure 4.8).

Figure 4.9 shows that during the falling process, both vortices touch, forming elongated vortex rings. In their descent the rings form a series of puffs 

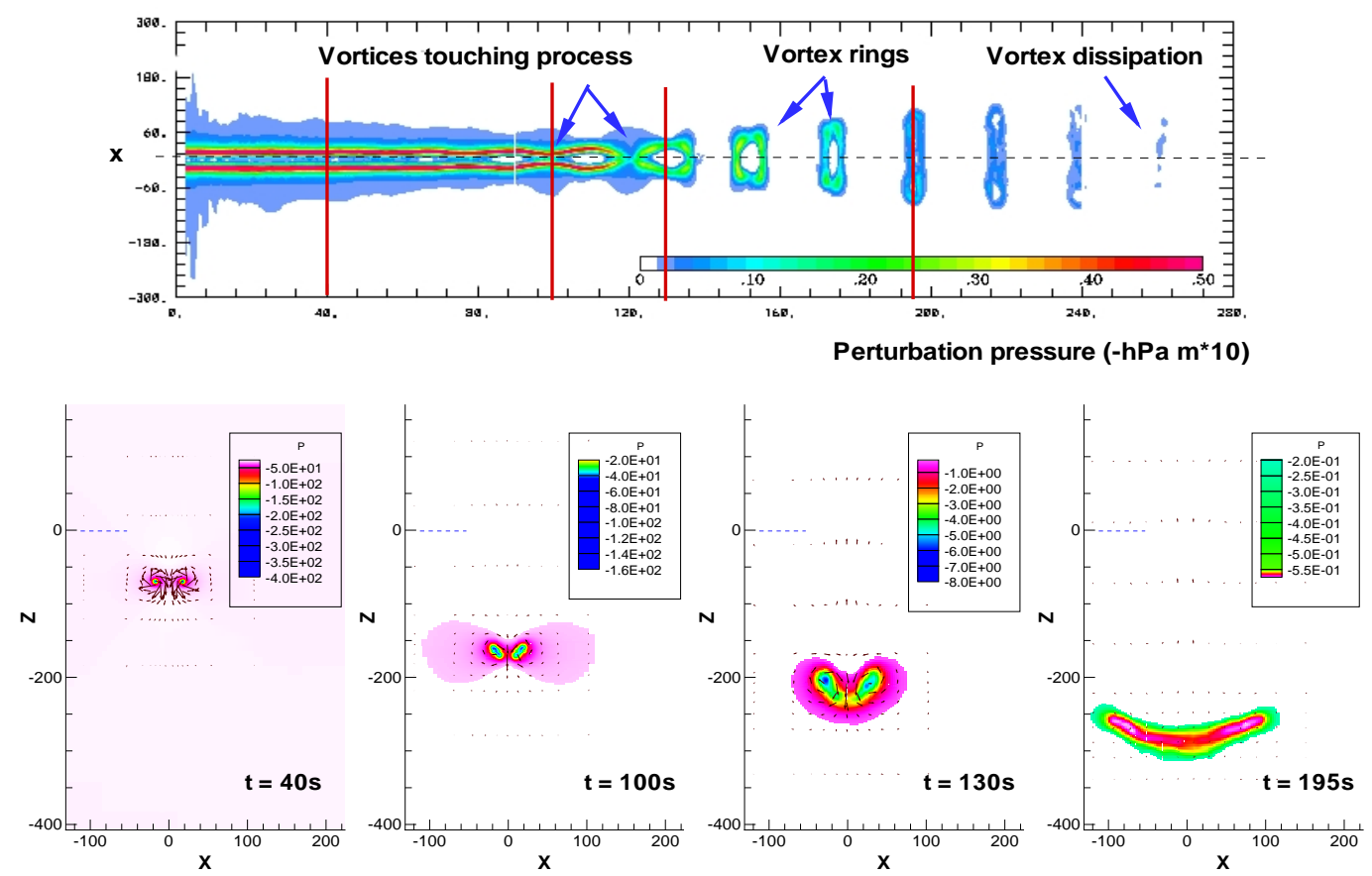

Figure 4.9: Contrail Simulation, 3D - Young Contrail - Perturbation Pressure - Vertical Integrated Drift Plot (top) and Down-stream averaged contours (bottom)

commonly seen in contrail evolution (Figure 4.6). After the vortex system has mostly died out a strong Brunt-Väisälä oscillation in the plume driven by the positive buoyancy as consequence of the atmospheric stratification is observed. The increase in the turbulence generated by the oscillation increases the plume dispersion until it diminishes to the ambient atmospheric turbulence level, after one or two BruntVäisälä periods ( 10-20 min).

\subsubsection{Older Contrail Dynamics}

The fluid dynamics of older contrails is mainly driven by atmospheric turbulence, wind shear, gravity waves, buoyant forces, and precipitation. Specifically in the case without mean wind shear imposed in the simulation, the horizontal 


\section{Contrail Simulation 3-D}
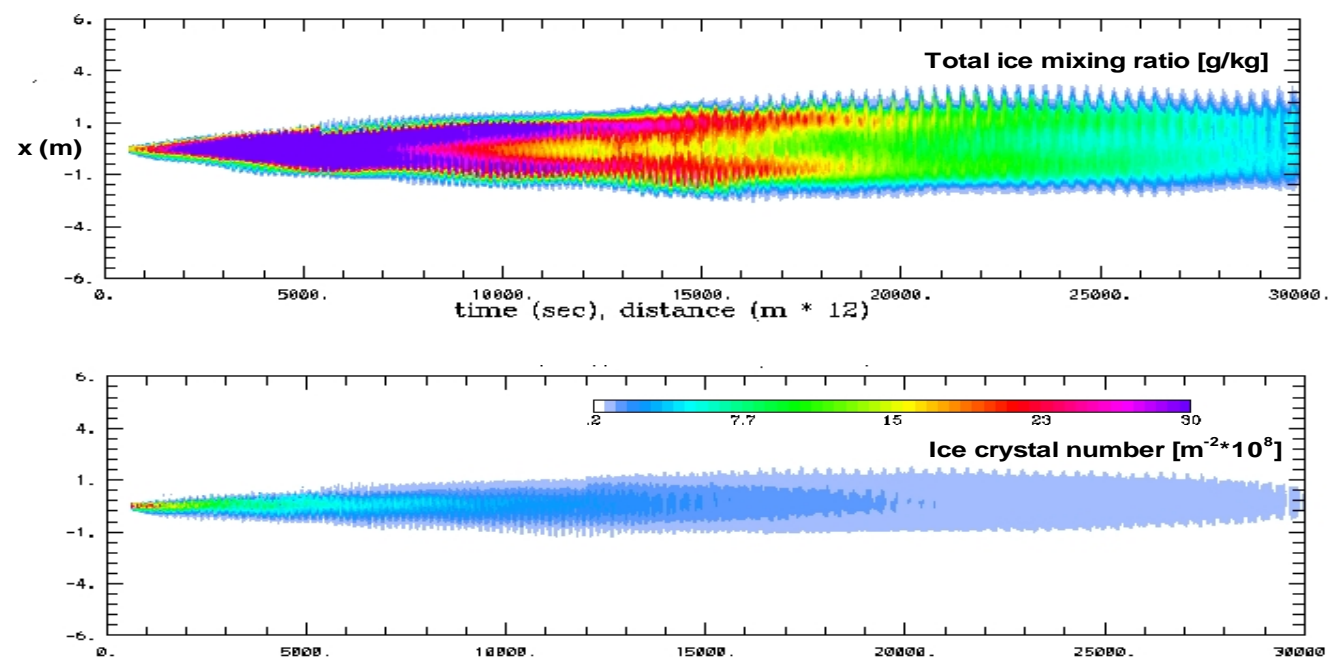

Figure 4.10: Contrail Simulation, 3D - Old Contrail - Vertical integrated ice mass mixing ratio (top) and number of ice crystals (bottom)

spreading observed in figure 4.10 depends on the level of atmospheric turbulence (producing local wind shear).

The remaining ice crystals from the compressional heating continue to be affected by the Kelvin effect but in minor degree, because the ice crystals have increased in size. D. C. Lewellen in a non-published report to Boeing defined this as "in situ loss", the mechanism of evaporation of the smallest ice crystals by the Kelvin effect, having their masses consumed by the largest ones, preferably in regions of high concentration of ice particles (at flight level).

When ice crystals have grown large enough to acquire adequate fall velocity they precipitate. The large ice particles fall eventually to subsaturated regions where they evaporate. This loss of ice particles is called "precipitation loss". 


\section{Contrail Simulation 3-D}

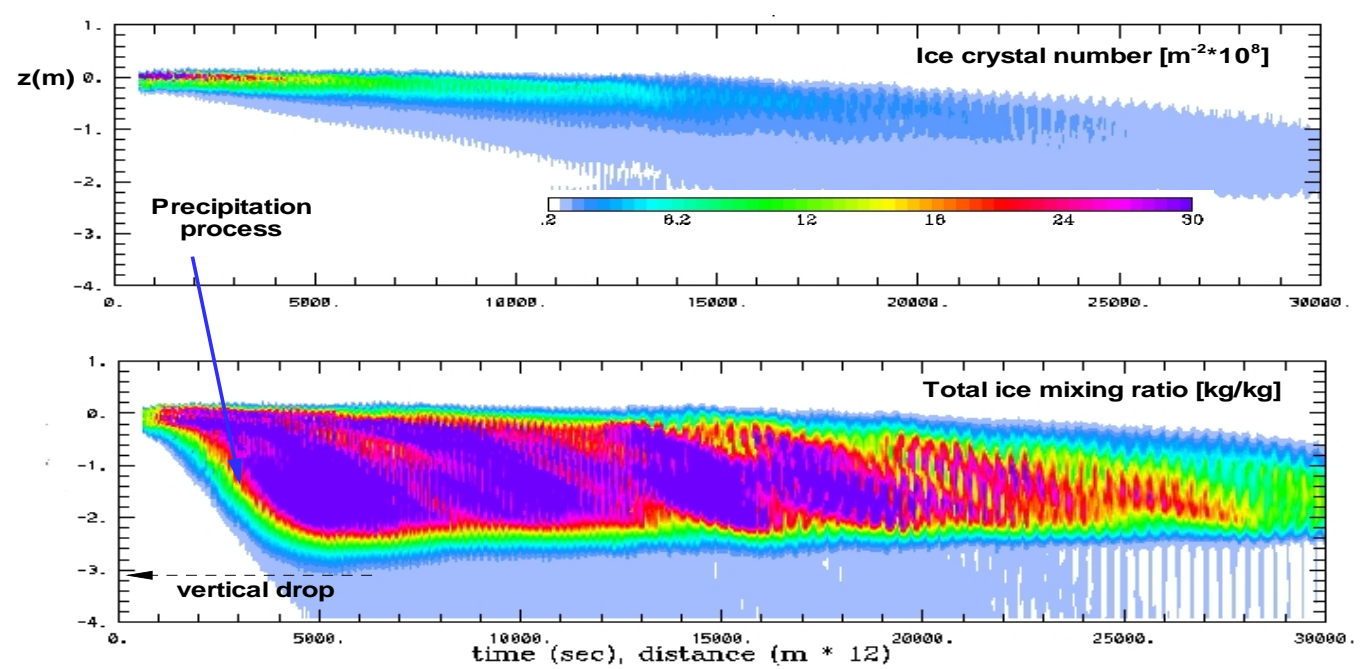

Figure 4.11: Contrail Simulation, 3D - Old Contrail - Cross-stream integrated ice mass mixing ratio (top) and ice crystal number (bottom)

Figure 4.11 shows the beginning of precipitation loss, which depends on the level of moisture, temperature, level of turbulence, and ice habit (capacitance, ventilation factor and fall velocity).

\subsection{Three-Dimensional versus Quasi-2D Simu-}

\section{lations}

Quasi-2D simulation is a very useful derivation of fully 3D simulation that includes some of the important physics involved in the vortex lifetime and determining the fall distance. The interaction with the ambient stratification and a representation of small-scale 3D mixing are included, but not some other ef- 
fects, in particular the Crow instability, due to the modest domain extent in the downstream direction. Previous WVU studies concluded that Q2D simulation is a good approximation to simulate contrail evolution. The advantages over the 3D-simulations are that they may be performed on a single ( and much smaller) grid, requiring less storage and computational time. This feature allows one to vary all parameters involved in the contrail evolution at early and late time. The grids are adjusted according with the contrail dynamic evolution, mainly the vortex system lifetime (vortex falling, vortices touching and linking, ring formation and break up).

In order to investigate the shape effects on contrail we used the Q2D model, which allows us to run a large set of simulations. The Q2D simulations were realized in two stages 1) simulation set out to $1000 \mathrm{~s}$ on domains of $1.6 \mathrm{~km}$ deep, $1.5 \mathrm{~km}$ wide $\mathrm{s}$ with finest cross-stream resolution of $6.7 \mathrm{~m}$ downstream and $1 \mathrm{~m}$ cross-stream; and 2) simulation sets with a starting point at 1000s on domains of $5 \mathrm{~km}$ deep, $12 \mathrm{~km}$ wide with finest resolution of $40 \mathrm{~m}$ downstream and $20 \mathrm{~m}$ cross-stream. To validate the level of accuracy of the Q2D model with respect to 3D simulation we have performed an uncertainty study of first order. A principle component of the uncertainty in the Q2D results is the the level of dispersion produced by running the simulations with different turbulence realizations for some conditions. The level of dispersion was calculated by a simple standard deviation with respect to the average value. It is useful and give us an important measure of the quality or significance of the numerical results. The statistical estimate of the dispersion in the Q2D results was obtained using 7 different turbulent realizations. Every realization consists in the same simulation, including all parameters and default setting, except for the initial random perturbation in the initial field. 

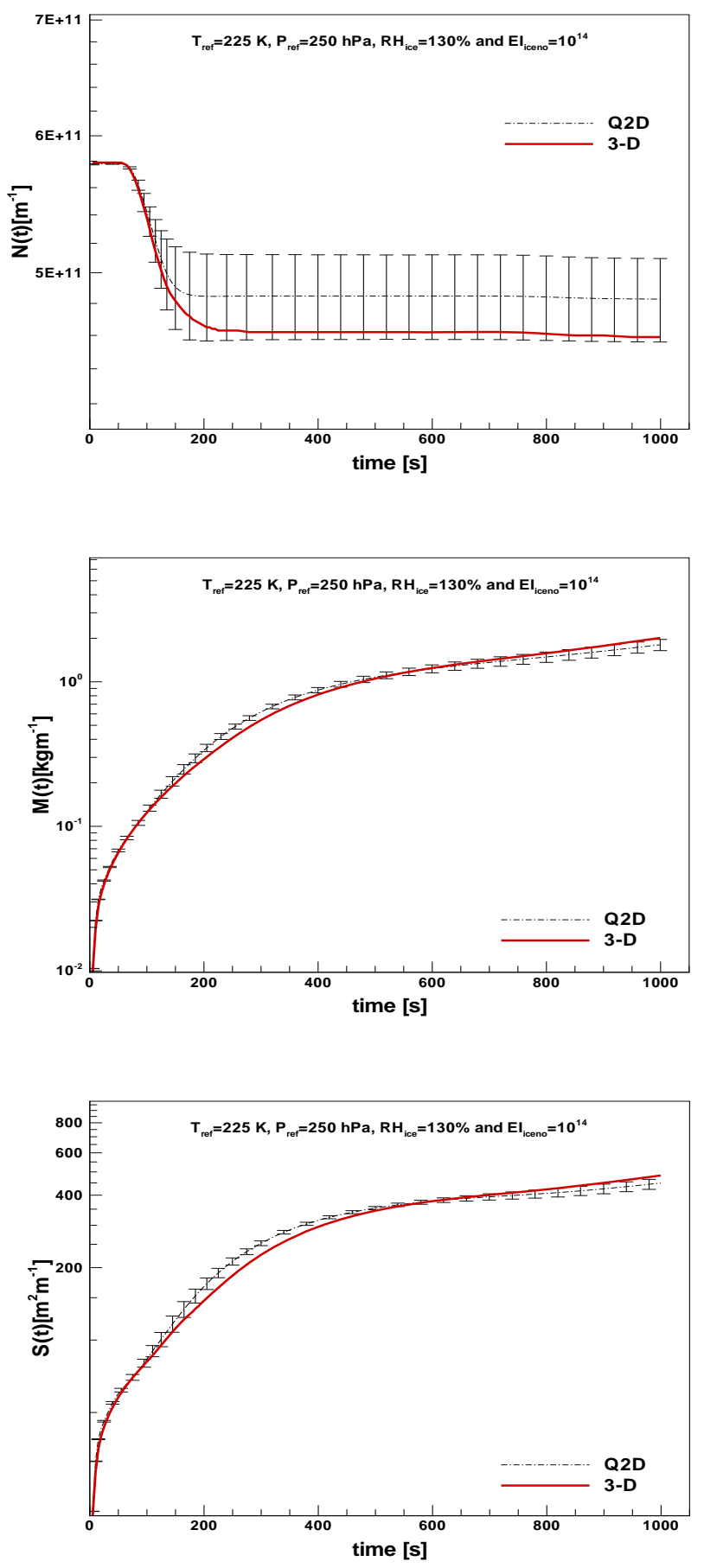

Figure 4.12: 3D and Q2D Comparison at early time - Base case I: N(t) (left), M(t) (middle), and $\mathrm{S}(\mathrm{t})$ (right) 

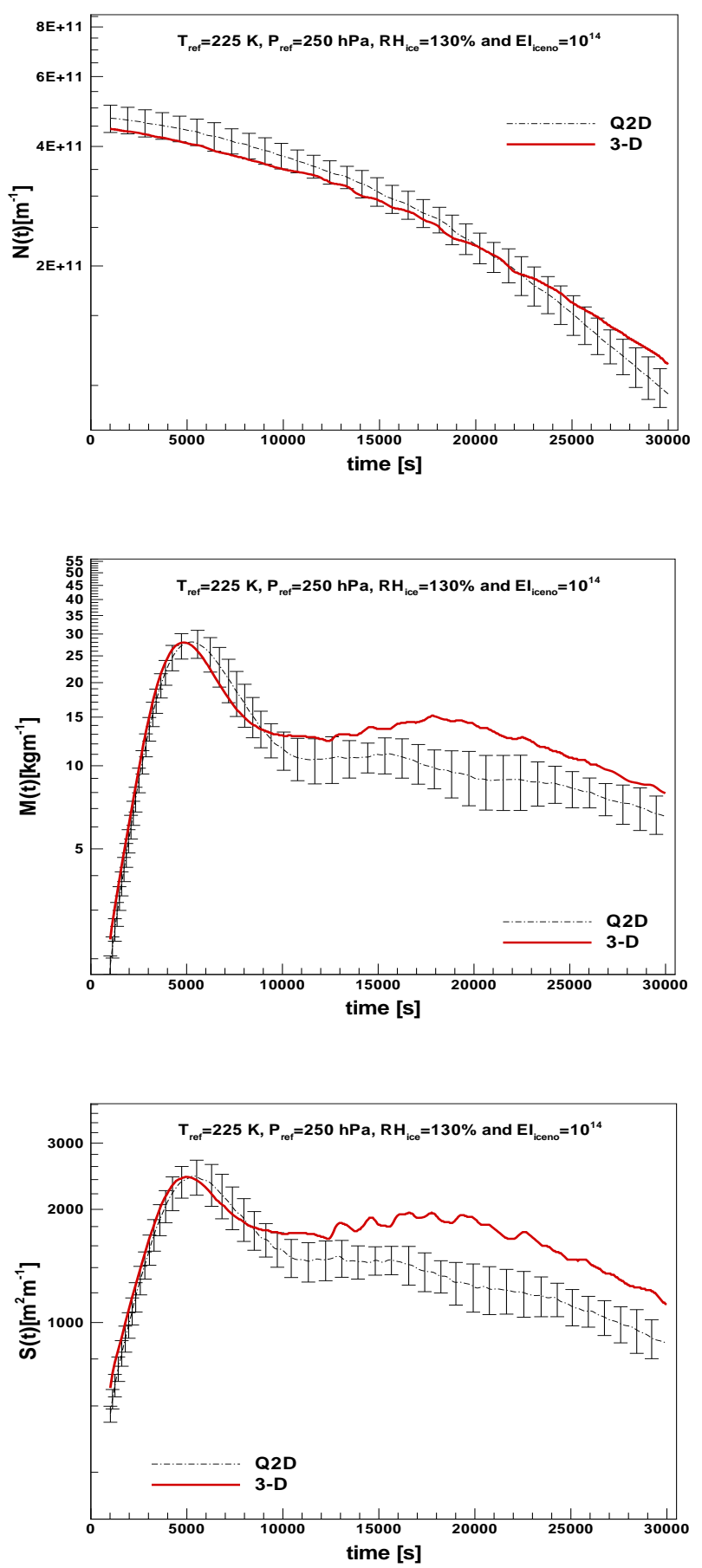

Figure 4.13: 3D and Q2D Comparison at late time - Base case I: N(t) (left), M(t) (middle), and $\mathrm{S}(\mathrm{t})$ (right) 
The 3D-Q2D contrail comparison was performed on the base that the turbulence fields matched between the 3D and Q2D cases. This critical task consisted in to find a very close level of dispersion in the Q2D contrail plume that match the 3D dispersion. The non-similar level of dispersion in the cases introduces certain level of uncertainty in the comparison process.

Figures 4.12 and 4.13 compare the Q2D and 3D results for young and older contrails, respectively. The specific case corresponds to the base case I, with a large level of ambient turbulence added every $10 \mathrm{~min}$. The results show good agreement between the models. The error bars show the level of dispersion in the Q2D results from different turbulent realizations, and the dashed-line is the Q2D average value. In young contrails and for the base case I (figure 4.12), the maximum value of the dispersion in $\mathrm{N}(\mathrm{t})$ relative to the average value is less than $6 \%$. The 3D results shown in red fall inside of the range of $\mathrm{Q} 2 \mathrm{D}$ dispersion and the difference from the Q2D average is less than the level of uncertainty. In the ice mass $\mathrm{M}(\mathrm{t})$ and ice surface area $\mathrm{S}(\mathrm{t})$ the maximum values of the dispersion relative to the average value are $9 \%$ and $8 \%$ respectively.

At late time, the level of dispersion increases as a consequence of the precipitation process and the turbulent distortions of the plume volume. The maximum values of Q2D spread in this specific case are: $12.29 \%, 23.32 \%$, and $15.11 \%$ for $\mathrm{N}(\mathrm{t}), \mathrm{M}(\mathrm{t})$ and $\mathrm{S}(\mathrm{t})$ respectively. The $3 \mathrm{D}$ results are in good agreement with the spreading Q2D results during the first 10000 s, after that time the difference between the $3 \mathrm{D}$ results and the Q2D average values increase, except in the $\mathrm{N}(\mathrm{t})$. This difference in the contrail metrics might be partly explainable by the difference in the level of dispersion of ice particles in the plume. In the 3D model the ice particles are more dispersed and hence the increase in mixing helps to preserve more ice crystals (figure $4.13 \mathrm{~N}(\mathrm{t})$ ). The preservation of more ice crys- 
tals increases the ice mass and ice surface area. The small level of spreading of the different realizations and the modest differences in the ice metrics between the Q2D and 3D results suggest that the Q2D model is adequate for this study. 


\section{Chapter 5}

\section{Shape Effect on Aircraft}

\section{Contrails}

The inclusion of hexagonal column, hexagonal plate and bullet rosette crystal shapes in the contrail code currently permits us to study shape effects on contrail evolution. This is an initial study to obtain in a realistic manner the microphysical and dynamical evolution of young and older contrails. An adequate understanding of the shape parameters (capacitance, fall velocity, ventilation factor, Kelvin effect, etc) is necessary to predict the microphysical properties of the contrail particles and their role in a real contrail. This study has been directed towards shape effects on the number of ice crystals surviving $N(t)$, ice mass $M(t)$, and total ice surface area $S(t)$ in the plume.

\subsection{Shape Effect Analysis}

In order to analyze the shape effect on contrail simulations, we have compared the average values and single realizations for every shape under study (hexagonal columns, hexagonal plates, bullet rosettes - 6 bullets and combination of shapes) 
compared to spheres. The study was focused on both young and older contrails. Contrail evolution is strongly affected by the initial configuration of the case under study (atmospheric conditions, initial number of ice crystals, size range spectrum, ice shape, etc). Young contrails (less than a half hour) are mainly composed of a high concentration of small particles. The small ice crystals are close enough to spheres that the shape effect should be negligible.

The evolution of contrail plumes is influenced greatly by the reference temperature and relative humidity with respect to ice at flight level. The reference temperature is important since it influences the air density $\rho$ and mainly the saturation mixing ratio $q_{s}$ that is directly related with the local relative humidity. The available amount of moisture in the ambient where the plume evolves is the key factor in the ice particles growth or evaporation rate.

\subsubsection{Young Contrails}

The variation of reference temperature and level of moisture are shown together in a figure to summarize our base case simulations. The results of base cases I (table 4.3) are presented in figures 5.1 and 5.2.

In young contrails, the results of the inclusion of non-spherical effects produce only modest changes in the contrail development. The difference due to the shape effect in the number of ice crystals $\mathrm{N}(\mathrm{t})$ and ice mass $\mathrm{M}(\mathrm{t})$ are not large compared with the uncertainty level. However, the ice surface area $\mathrm{S}(\mathrm{t})$ differences are more significant, driven mainly by geometrical rather than dynamical effects.

In order to visualize the difference between the shapes, a useful parameter was defined "Maximum difference". It was calculated by a simple difference respect 

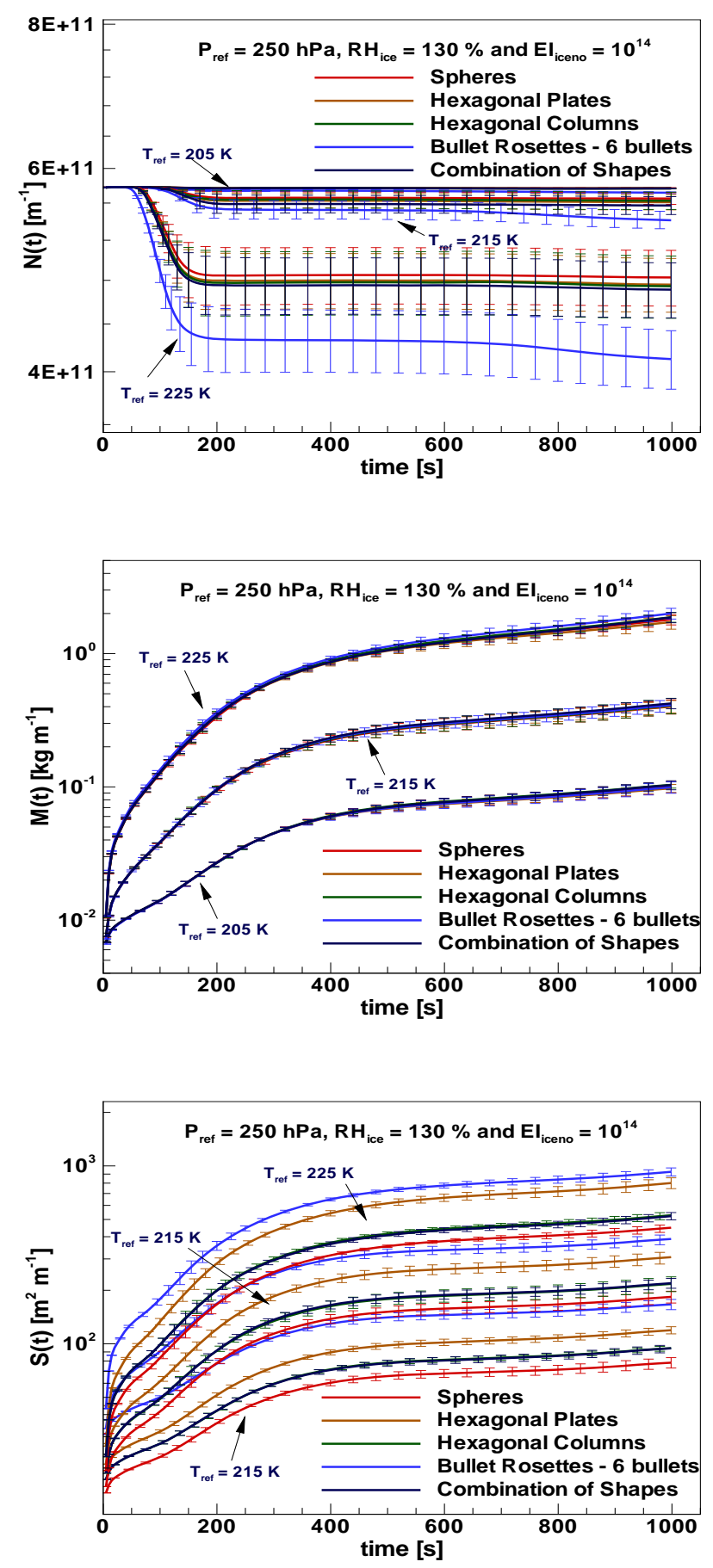

Figure 5.1: Comparison of all shapes. $\mathrm{N}(\mathrm{t}), \mathrm{M}(\mathrm{t})$, and $\mathrm{S}(\mathrm{t})$ - Average values, case I, $T_{\text {ref }}$ variation at early time 

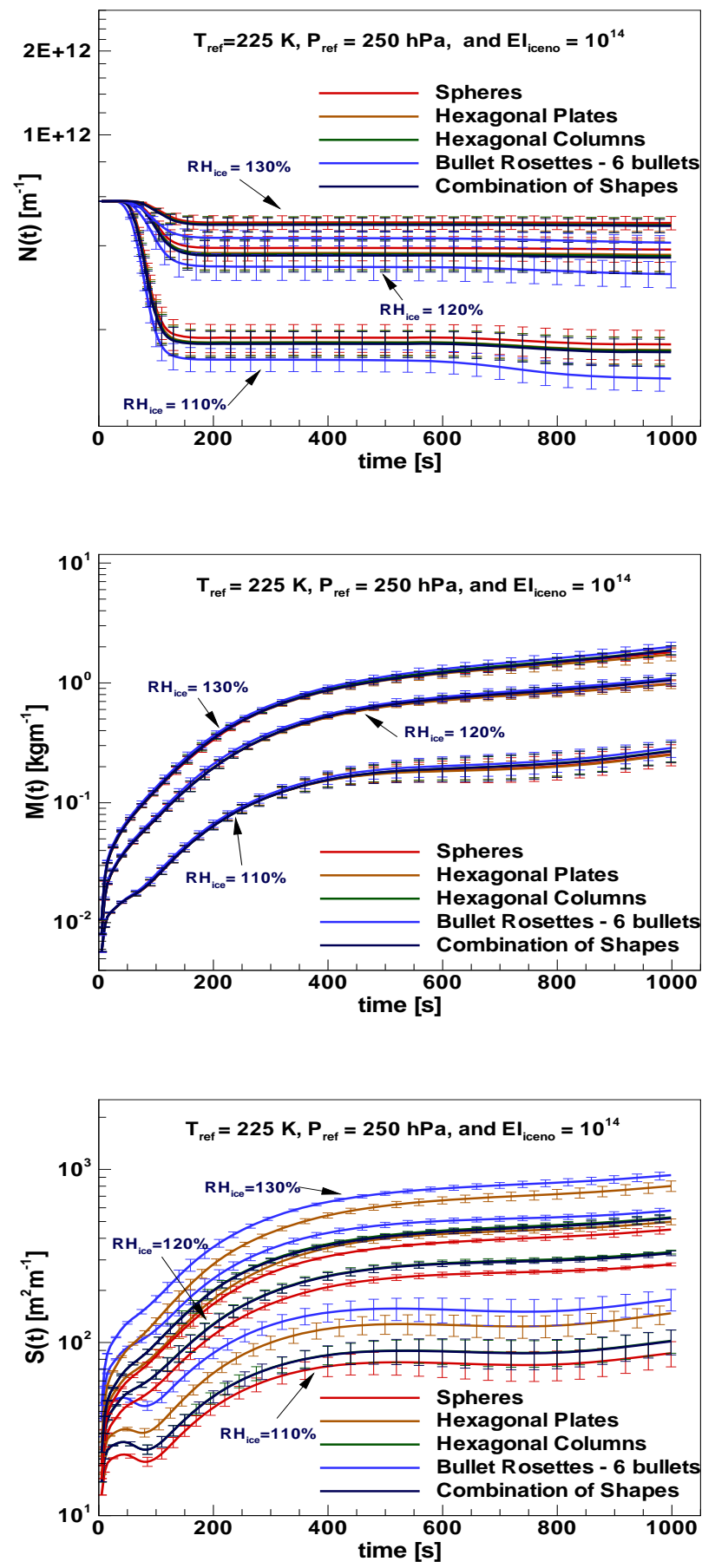

Figure 5.2: Comparison of all shapes. $\mathrm{N}(\mathrm{t}), \mathrm{M}(\mathrm{t})$, and $\mathrm{S}(\mathrm{t})$ - Average values, case I, $R H_{\text {ice }}$ variation at early time 
to spherical results and divided by spherical results. The maximum difference values in the ice metrics are presented in table 5.1.

Table 5.1: Maximum shape difference for $T_{\text {ref }}$ and $R H_{\text {ice }}$ variation - Base cases I young contrails

\begin{tabular}{llll}
\hline Base Cases I & $\mathrm{N}(\mathrm{t})$ & $\mathrm{M}(\mathrm{t})$ & $\mathrm{S}(\mathrm{t})$ \\
\hline$T_{\text {ref }}$ & $2.4 \%$ & $5.6 \%$ & $68 \%$ \\
\hline$R H_{i c e}$ & $6.5 \%$ & $7.8 \%$ & $34 \%$ \\
\hline
\end{tabular}

The difference in the ice metrics due to shape effect is mainly driven by the Kelvin effect term in the ice mass growth rate equation (equation 3.1). The growth rate involves parameters directly related to the ice crystal shape, and the atmospheric conditions. The perfect conditions for the influence of the Kelvin effect is observed at early time (see figure 3.2), mainly, at flight level where the contrails contain high population of small ice crystals. The Kelvin effect reduces the growth rate of the smaller ice particles of any shape which fall victim to evaporation in the adiabatic heating process in the falling vortex system. The difference in the ice metric $\mathrm{N}(\mathrm{t})$ is directly related to the magnitude of the Kelvin effect. The increasing difference relative to spheres follows the order: hexagonal plates, combination of shapes, hexagonal columns and bullet rosettes. The small difference seen for different shapes (except bullet rosettes) in the $\mathrm{N}(\mathrm{t})$ is due to the smaller ice particles in the initial spectrum being defined as small hexagonal columns with aspect ratio 1 , that is close to spheres.

The results for bullet rosette - 6 shapes differ significantly from those with spherical particles, showing greater loss of ice crystals due to the high value of the Kelvin effect term in the ice mass growth rate. However, this is an unphysical case, since very small ice crystals with well defined complex shapes have not been found in in-situ measurement. We include the results only for 
illustrative purposes.

In the case of relatively small ice crystals, the in-situ loss of ice crystals plays a significant role in the number of ice crystals remaining in the plume. The difference due to the shape effect is mainly driven by the Kelvin effect as seen in table 5.2 .

Table 5.2: Maximum shape difference for $T_{\text {ref }}$ and $R H_{\text {ice }}$ variation - Base cases II young contrails

\begin{tabular}{llll}
\hline Base Cases I & $\mathrm{N}(\mathrm{t})$ & $\mathrm{M}(\mathrm{t})$ & $\mathrm{S}(\mathrm{t})$ \\
\hline$T_{\text {ref }}$ & $22.2 \%$ & $4.3 \%$ & $28.4 \%$ \\
\hline$R H_{\text {ice }}$ & $22.2 \%$ & $4.5 \%$ & $58 \%$ \\
\hline
\end{tabular}

The results of base case II (reference temperature and level of moisture variation; (table 4.3) are shown in figures 5.3 and 5.4.

Similarly as in the base case I, the ice mass $M(t)$ and ice surface area $\mathrm{S}(\mathrm{t})$ present the same trends with shape. Independent of the crystal size, the differences seen in ice surface area $\mathrm{S}(\mathrm{t})$ are strongly influenced by the non-spherical effects. However, an inverse behavior is observed in the ice crystal number $\mathrm{N}(\mathrm{t})$. The difference by the shape effect is larger compared with the level of dispersion. In the cases where the contrail plumes are formed mainly by relative small crystals, the level of uncertainty is very small.

\subsubsection{Older Contrails}

Older contrails can have a wide spectrum of morphology possibilities and lifetimes determined by atmospheric turbulence/wave state, shear levels, initial level of ice crystals, initial size range, atmospheric supersaturation, etc. The ice crystals around the contrail plume edges become large enough to acquire 

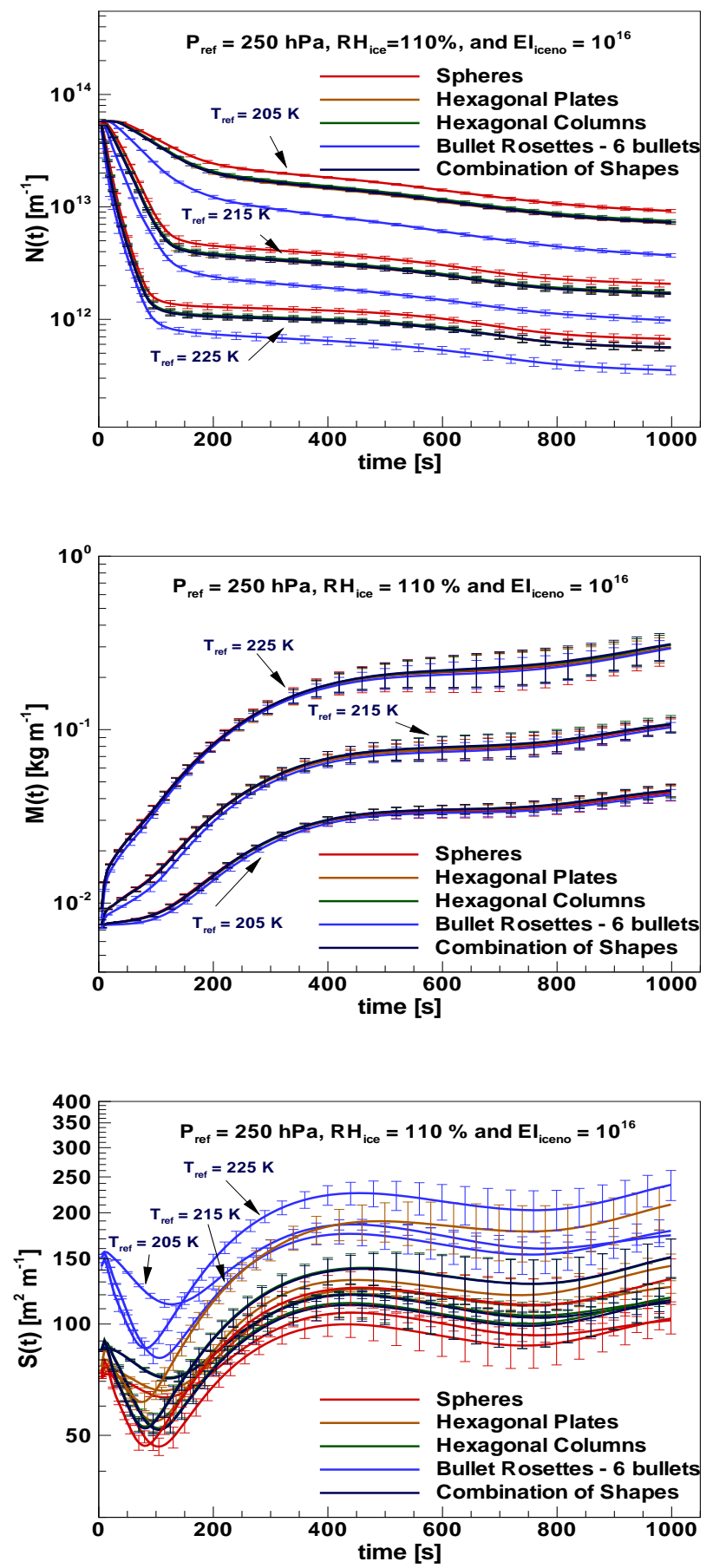

Figure 5.3: Comparison of all shapes. $\mathrm{N}(\mathrm{t}), \mathrm{M}(\mathrm{t})$, and $\mathrm{S}(\mathrm{t})$ - Average values, case II, $T_{\text {ref }}$ variation at early time 

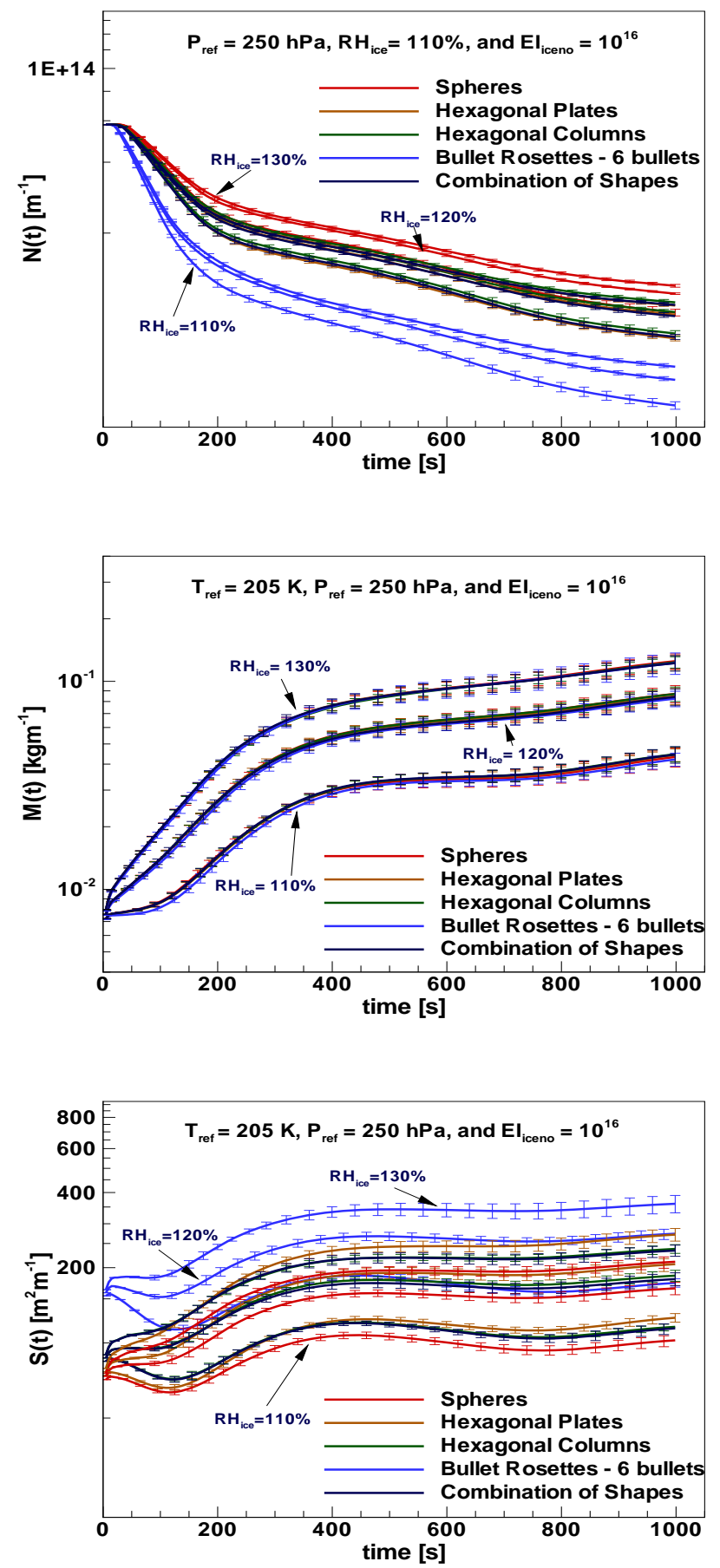

Figure 5.4: Comparison of all shapes. $\mathrm{N}(\mathrm{t}), \mathrm{M}(\mathrm{t})$, and $\mathrm{S}(\mathrm{t})$ - Average values, case II, $R H_{\text {ice }}$ variation at early time 
significant terminal velocities which can then fall into subsaturated air regions and sublime or fall out of the domain.

The results of late time simulations are strongly influenced by the results at early time. During the late time simulation the mixing process is reduced due to the vortex wake having died out. After this period, the phase of dispersion the ice crystals continue below the influence of the Kelvin effect driven by the atmospheric turbulence, atmospheric waves, and buoyancy forces. The low level of mixing cannot mitigate completely the ice crystals lost by the Kelvin effect. This geometrical effect is reduced as the ice spectrum is shifted toward the largest size crystals. At this point, the influence of the shape effect (fall velocities and growth rate) on the loss rate by precipitation reaches importance.

Figures 5.5, 5.6, 5.7, and 5.8 present the results on variation of reference temperature and level of moisture. As was discussed before, older contrail simulations (large or small particles) show the expected difference in the level of dispersion. In the base case I, with relative large crystals, the level of dispersion presents an overlapping evolution in the ice metrics between all shapes, except in the ice surface area $\mathrm{S}(\mathrm{t})$. However, again the shape effect is not large with respect to the uncertainty level. Figure 5.5 shows that in contrails formed mainly by large crystals the geometrical difference is more evident in the ice surface area $\mathrm{S}(\mathrm{t})$. Independent of atmospheric conditions during the last part of simulations with large crystals, a slightly lower average number of ice crystals $\mathrm{N}(\mathrm{t})$ are preserved by the hexagonal columns than hexagonal plates. This difference can be explained by the higher fall velocities of hexagonal columns compared with the slower falling shapes (e.g. hexagonal plates). In aged contrails the large crystals grow faster by the higher capacitance factor and by the Kelvin effect (at expenses of small ones). 

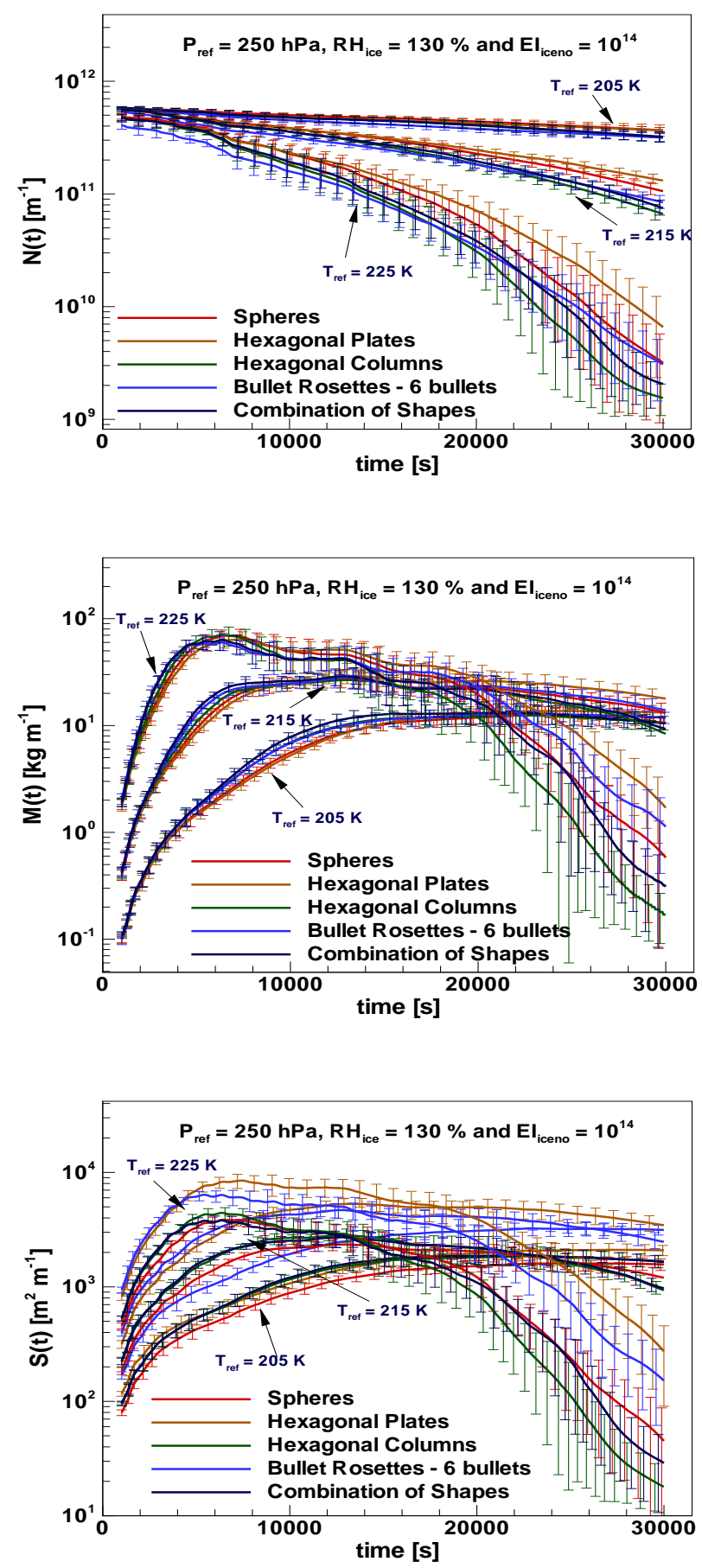

Figure 5.5: Comparison of all shapes. $\mathrm{N}(\mathrm{t}), \mathrm{M}(\mathrm{t})$, and $\mathrm{S}(\mathrm{t})$ - Average values, case I, $T_{\text {ref }}$ variation at late time 

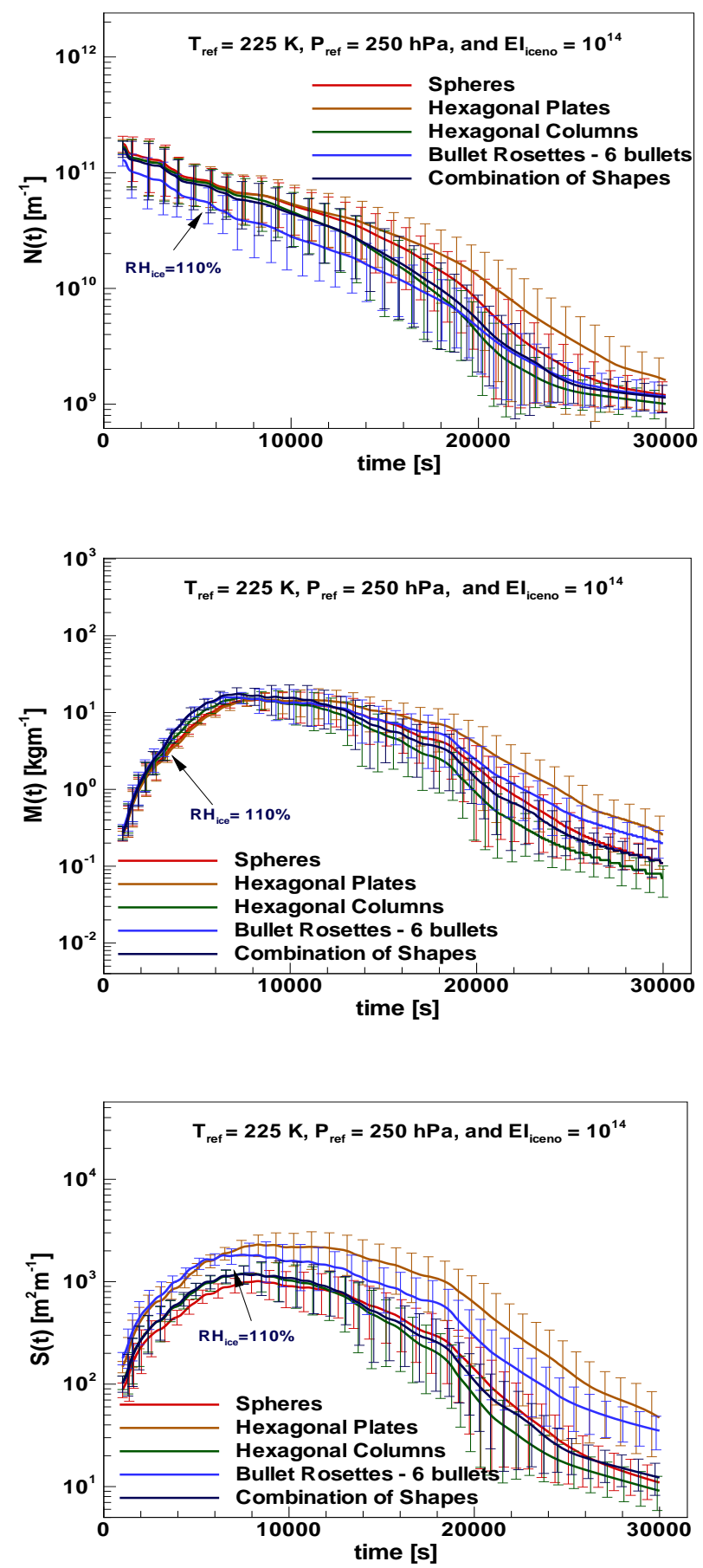

Figure 5.6: Comparison of all shapes. $\mathrm{N}(\mathrm{t}), \mathrm{M}(\mathrm{t})$, and $\mathrm{S}(\mathrm{t})$ - Average values, case I, $R H_{\text {ice }}=110 \%$ variation at late time 

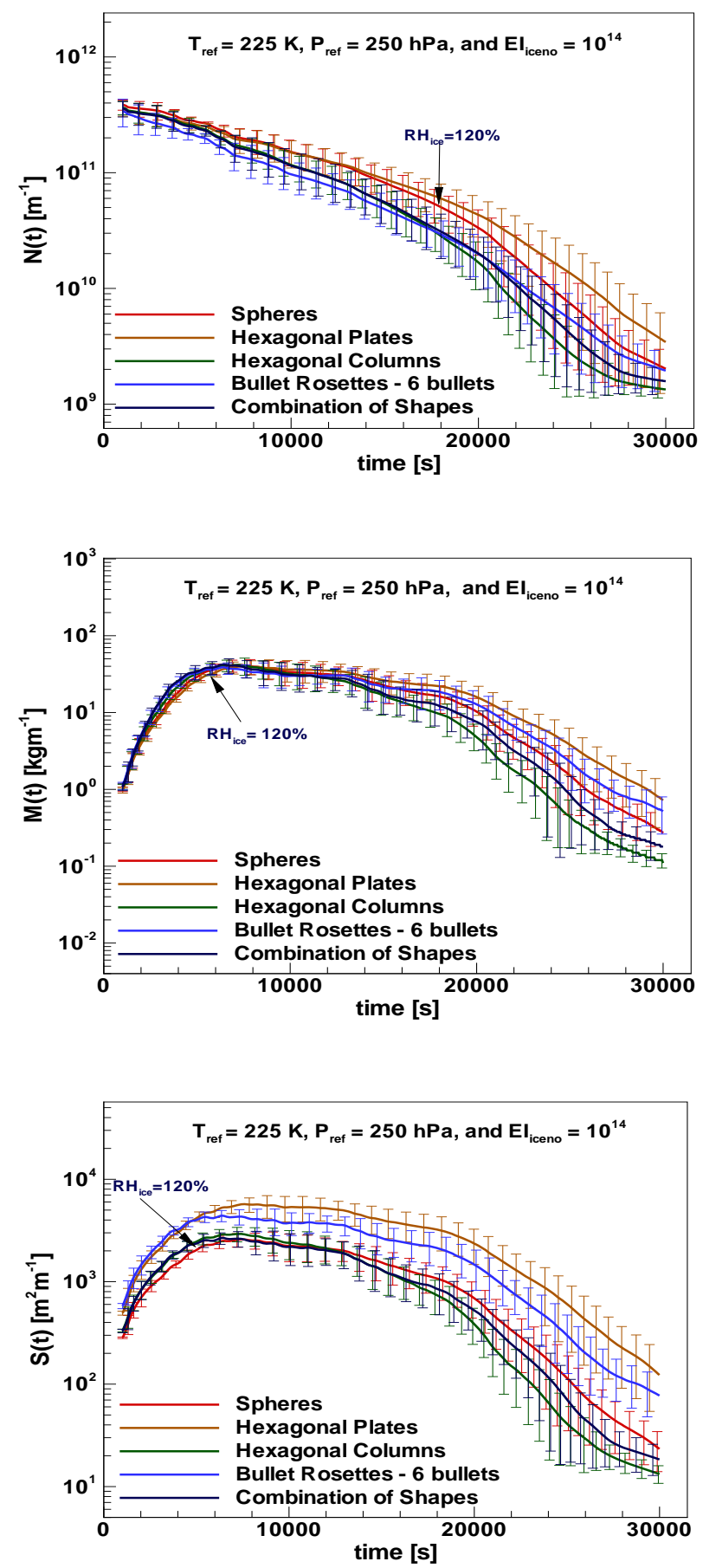

Figure 5.7: Comparison of all shapes. $\mathrm{N}(\mathrm{t}), \mathrm{M}(\mathrm{t})$, and $\mathrm{S}(\mathrm{t})$ - Average values, case I, $R H_{\text {ice }}=120 \%$ variation at late time 

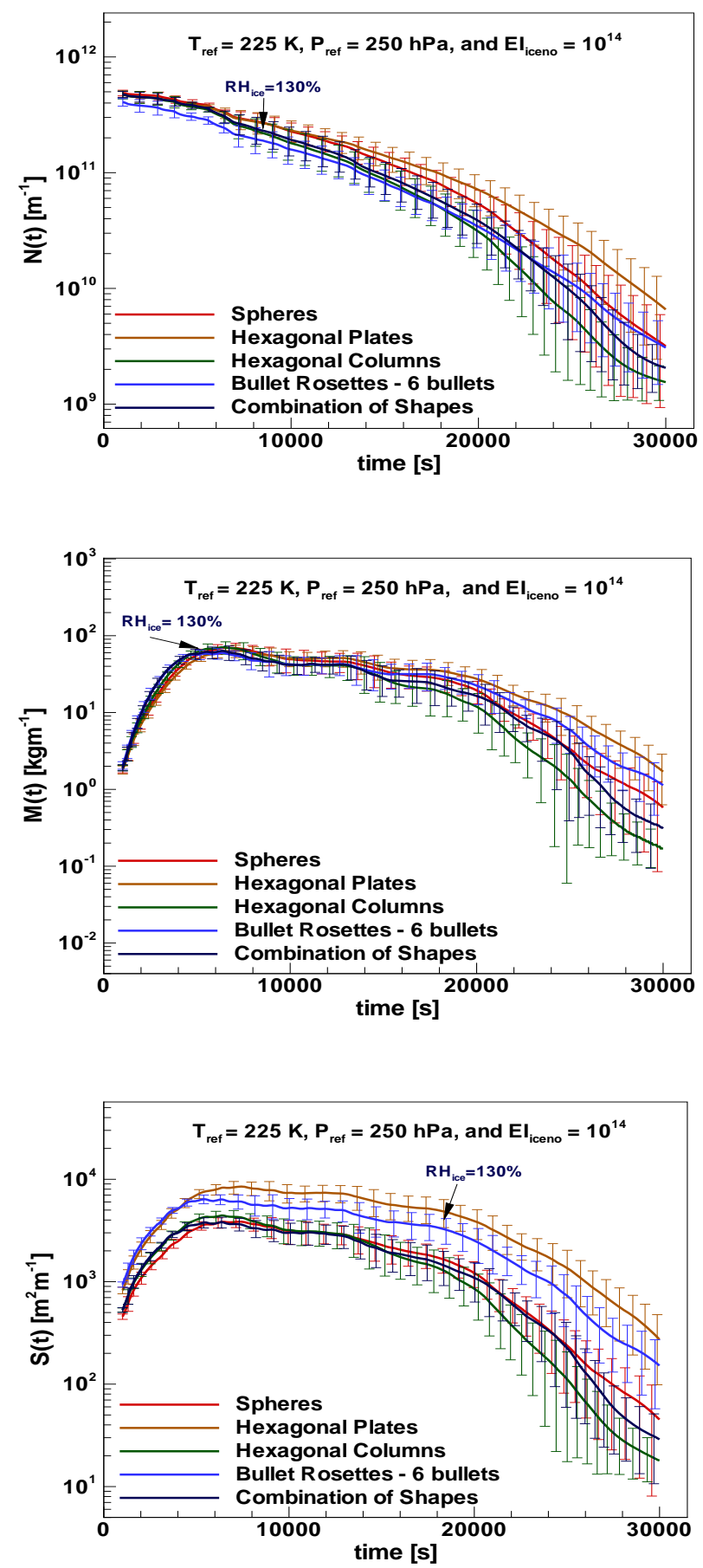

Figure 5.8: Comparison of all shapes. $\mathrm{N}(\mathrm{t}), \mathrm{M}(\mathrm{t})$, and $\mathrm{S}(\mathrm{t})$ - Average values, case I, $R H_{\text {ice }}=130 \%$ variation at late time 
The small differences observed in the ice mass $M(t)$ average results suggest a negligible influence of the shape effects for any $T_{r e f}$ and $R H_{\text {ice }}$ level.

Even though the parameterization is inconsistent in the fall velocity of the larger ice crystals (figure 3.8), the results in the number of ice crystals $\mathrm{N}(\mathrm{t})$ and ice crystal mass $\mathrm{M}(\mathrm{t})$ show an insensitivity to the fall velocity differences. In order to have correct results in the ice metrics, a better approximation in the fall velocity for large ice particles is needed to include the high Reynolds correction of non-spherical shapes.

Figures 5.9 and 5.10 show the evolution of older contrails (base case II) formed by relatively small ice crystals. As in young contrails, the difference by the shape effect is larger than the level of uncertainty. In figure 5.9, the precipitation process is almost not observed in these contrails (except the case with high $T_{\text {ref }}$ because ice crystals are no longer "small"). The ice crystals loss is mainly by "in-situ loss". At late time, contrails formed by relative large and small ice crystals show that the shape effect difference is independent of the level of reference temperature and relative humidity with respect to ice.

An important observation in the results is that cases of relative small and large ice crystals show an important difference in ice crystal surface area by meter of flight path $\mathrm{S}(\mathrm{t})$. This difference is not consequence of the contrail dynamics, since the ice crystals number remaining in the plume presents very close results. An explanation for this difference is that ice surface area is sensitive to the ice shape and the number of ice crystals, mainly to the shape effect. In chapter 3 was shown that there is significant differences between the ice surface areas of all shapes in study (figure 3.5). The difference in $\mathrm{S}(\mathrm{t})$ is clearly observed in young and older contrails. In older contrails formed by large crystals is more notorious this difference than small ones. Based in the results, we can conclude 

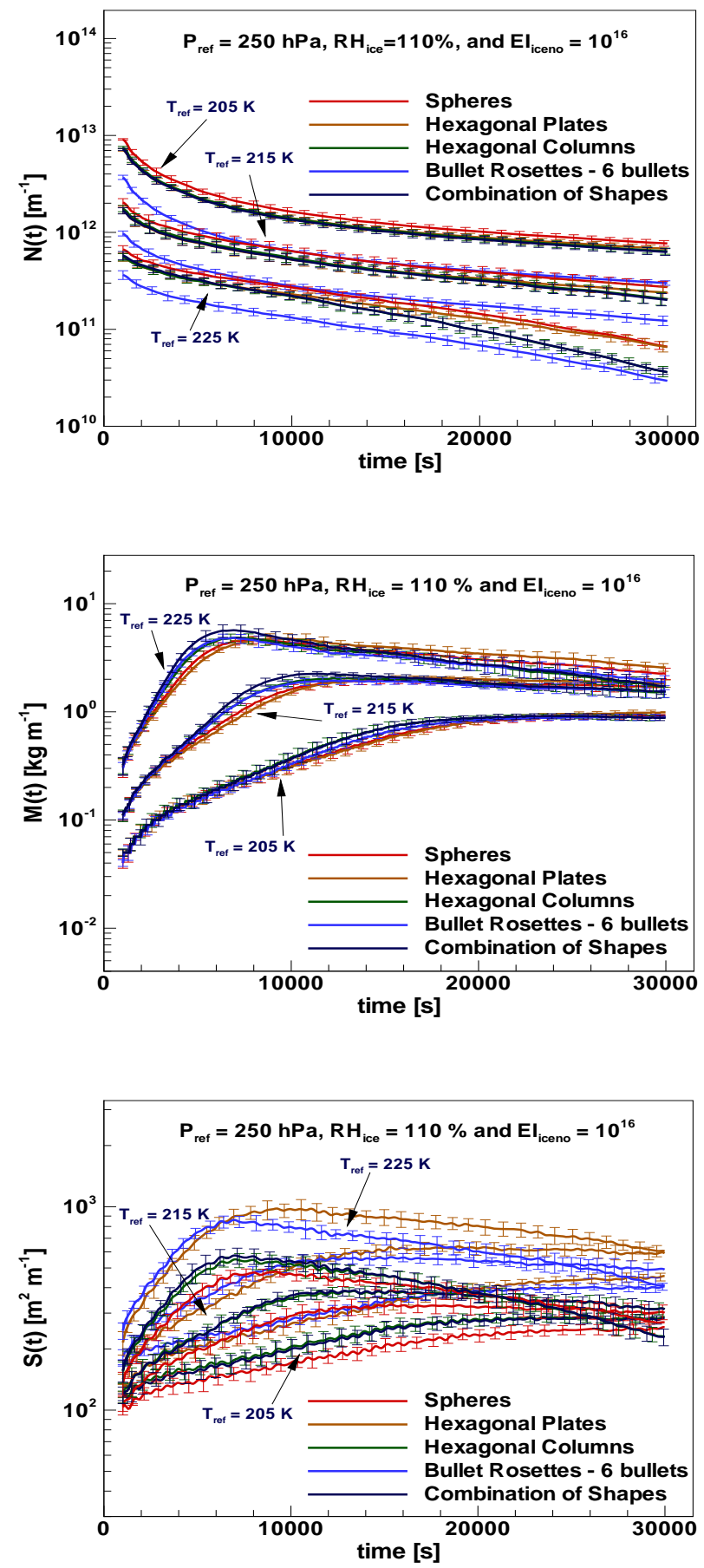

Figure 5.9: Comparison of all shapes. $\mathrm{N}(\mathrm{t}), \mathrm{M}(\mathrm{t})$, and $\mathrm{S}(\mathrm{t})$ - Average values, case II, $T_{\text {ref }}$ variation at late time 

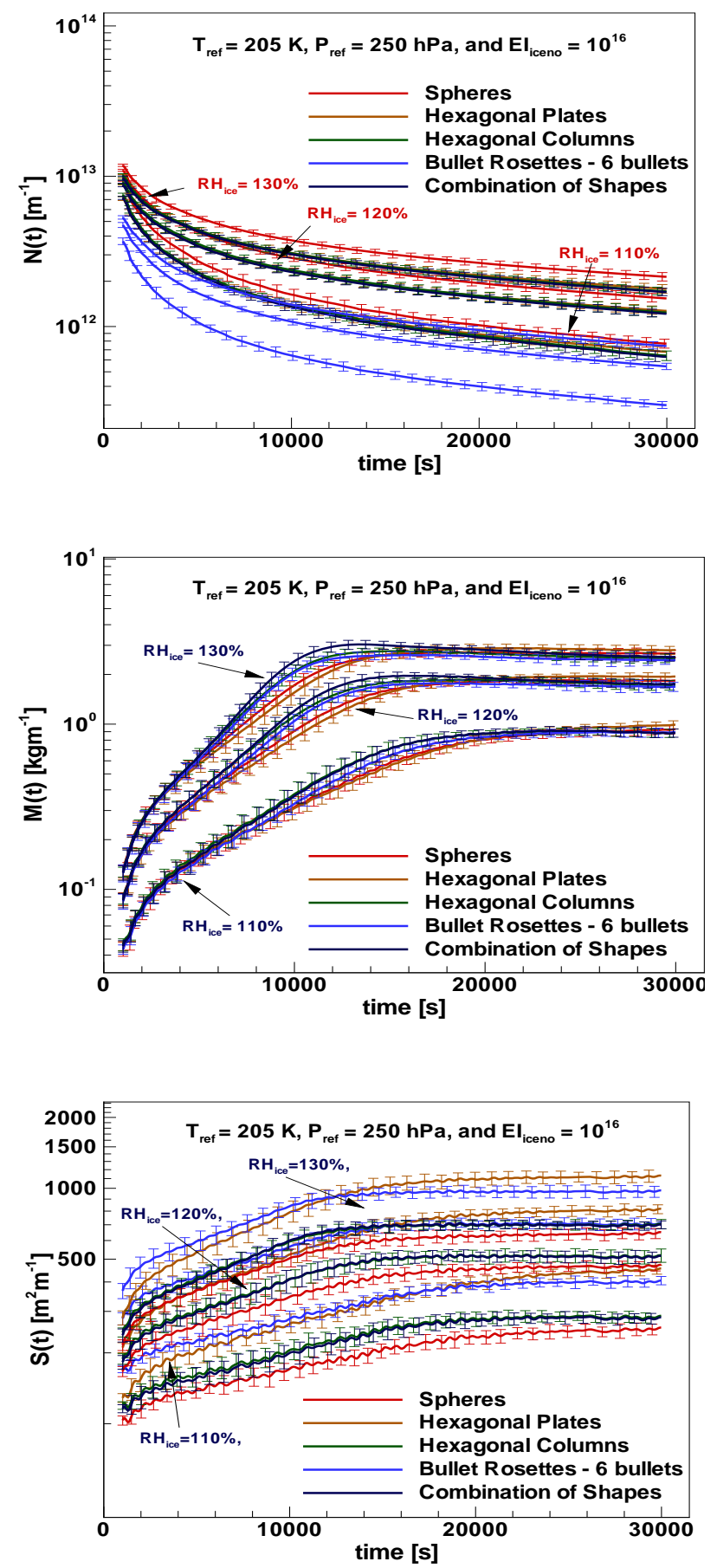

Figure 5.10: Comparison of all shapes. $\mathrm{N}(\mathrm{t}), \mathrm{M}(\mathrm{t})$, and $\mathrm{S}(\mathrm{t})$ - Average values, case II, $R H_{\text {ice }}$ variation at late time 
that shape sensitivity seen in $\mathrm{S}(\mathrm{t})$ is mostly geometrical rather than dynamical. The influence of cross-stream wind shear and varying stratification on the shape effect were also analyzed through single realizations. This type of comparison involves more uncertainty, but will be useful in the final conclusions.

Previous studies of contrails affected by wind shear in the cross-stream directions were reported by Lewellen and Lewellen (2001a). In young contrails, a weak level of wind shear only slightly disturbs the vortex dynamics, but changes the appearance of the plume presenting a little horizontal smearing. However, high levels of wind shear modify the evolution of the vortex pair, reducing the vertical displacement and decreasing the adiabatic heating. Additionally, an increase in the level of wind shear generally increases the mixing of ambient moisture into the contrail. In older contrails, the wind shear modifies the morphology of the plume, causing displacement and smearing in the horizontal direction.

The influence of the two levels of wind shear $\left(d u / d z=5 \times 10^{-4} \mathrm{~s}^{-1}\right.$ and $1 \times 10^{-3}$ $\mathrm{s}^{-1}$ ) were analyzed in base cases I and II (figures 5.11 and 5.12). The shape difference in the ice metrics $\mathrm{N}(\mathrm{t}), \mathrm{M}(\mathrm{t})$, and $\mathrm{S}(\mathrm{t})$ between all shapes with respect to spheres is consistent with the shape effect and maintains a level similar that previously observed in the $T_{r e f}$ and $R H_{i c e}$ analysis.

The shape differences observed in the ice metrics were expected and suggest that those trends are independent of the level of wind shear. The difference due to the shape effect below the influence of modest levels of wind shear and size range used in this study can be considered insignificant.

Additionally, we have also simulated a case with different level of atmospheric stratification. The value $5 \mathrm{~K} / \mathrm{km}$ was chosen only for the purpose of analyzing its influence in the shape effect. The ice metrics of base case I and II were compared in figures 5.13 and 5.14. In order to analyze the influence of this 

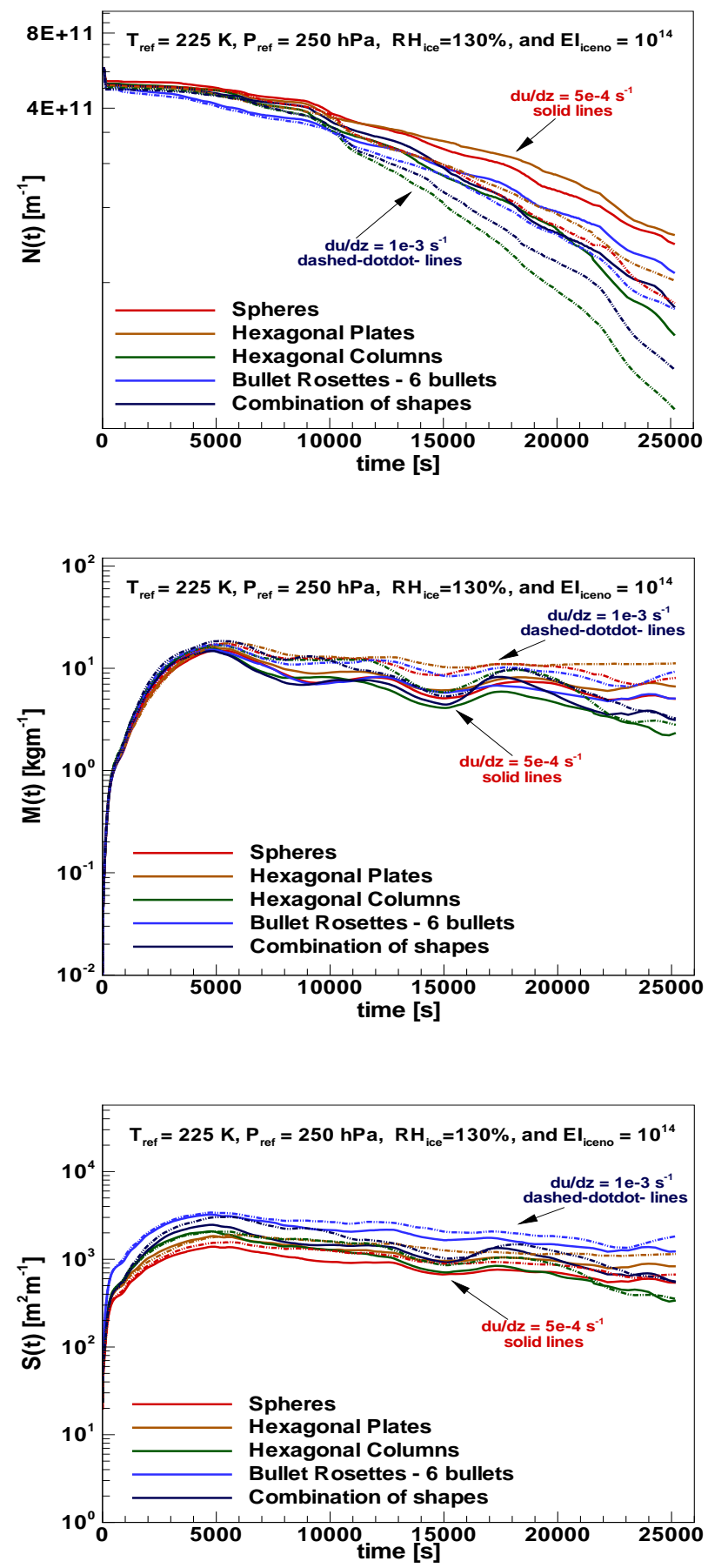

Figure 5.11: Comparison of all shapes. $\mathrm{N}(\mathrm{t}), \mathrm{M}(\mathrm{t})$ and $\mathrm{S}(\mathrm{t})$ - single realizations, wind shear at late time. Case I. 

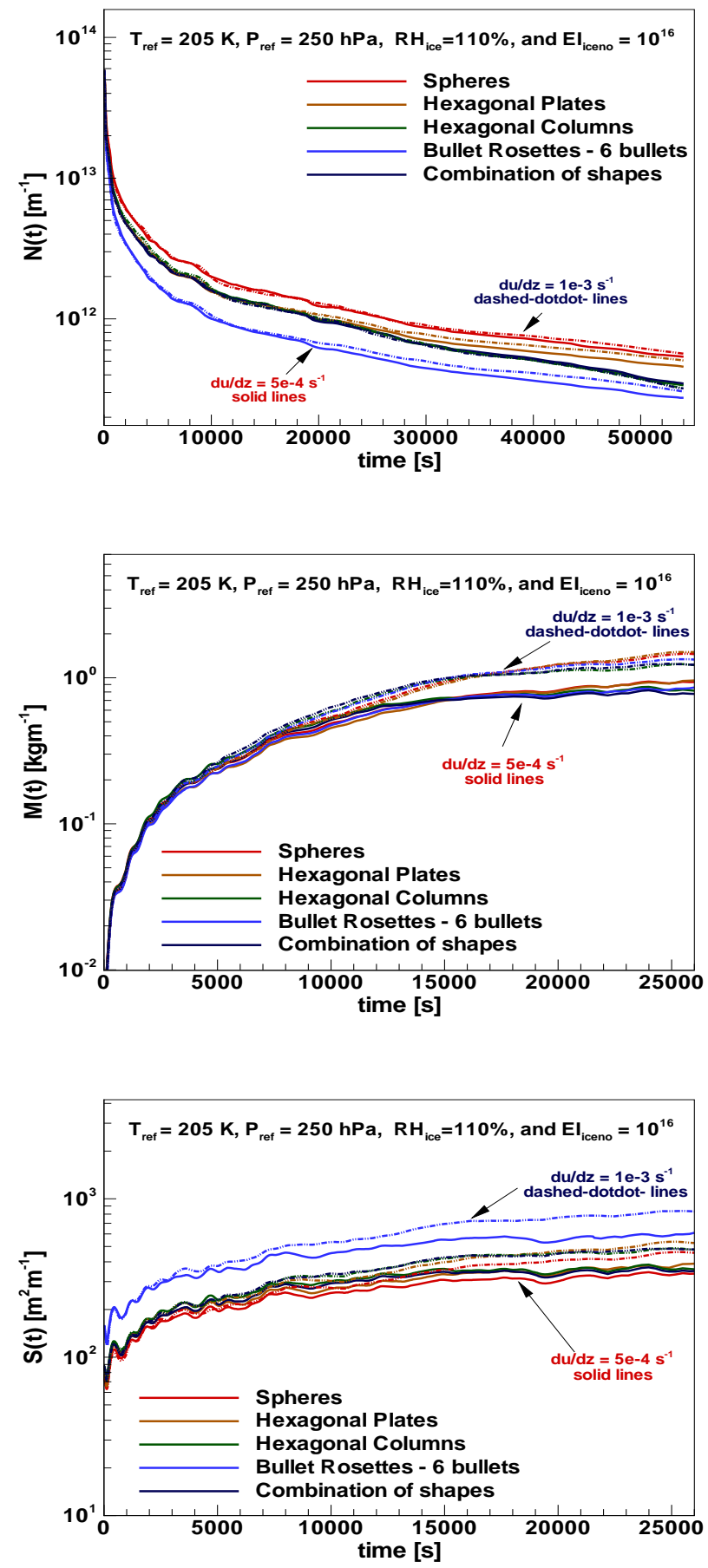

Figure 5.12: Comparison of all shapes. $\mathrm{N}(\mathrm{t}), \mathrm{M}(\mathrm{t})$ and $\mathrm{S}(\mathrm{t})$ - single realizations, wind shear at late time. Case II. 
parameter, it is relevant to know previous studies on ambient potential temperature stratification $d \theta / d z$ (Lewellen and Lewellen, 2001a; Huebsch and Lewellen, $2006 \mathrm{~b})$ which found that in young contrails a higher stratification level increases the detrainment, decreases the vertical plume size by reduction in the descent of the vortex system and hence diminishes the adiabatic heating process. The reduction in the adiabatic heating and the increase in the level of available moisture (that fights against the loss by Kelvin effect) permits more ice crystals to survive.

In older contrails and independently of the range size, the ice metrics $\mathrm{N}(\mathrm{t})$, $\mathrm{M}(\mathrm{t})$, and $\mathrm{S}(\mathrm{t})$ present similar levels of difference as a function of crystal shape as in the analysis of $T_{r e f}$ and $R H_{i c e}$.

Based in the results previously analyzed, the conclusion is that in young contrails the ice metric differences are affected by the shape effect but weakly. In older contrails, the shape difference is higher in cases where the precipitation process is present. This high difference is not larger than the level of uncertainty, reducing the significance of this difference. This study suggests that the difference due to the shape effect is independent of the atmospheric parameters involved in the analysis. The spherical ice crystal shape will represent very well all shapes found in in situ studies.

In young contrails, the shape effect can be considered negligible. The simulation of older contrails can be more consistent if it is continued using spherical results as a starting point. 

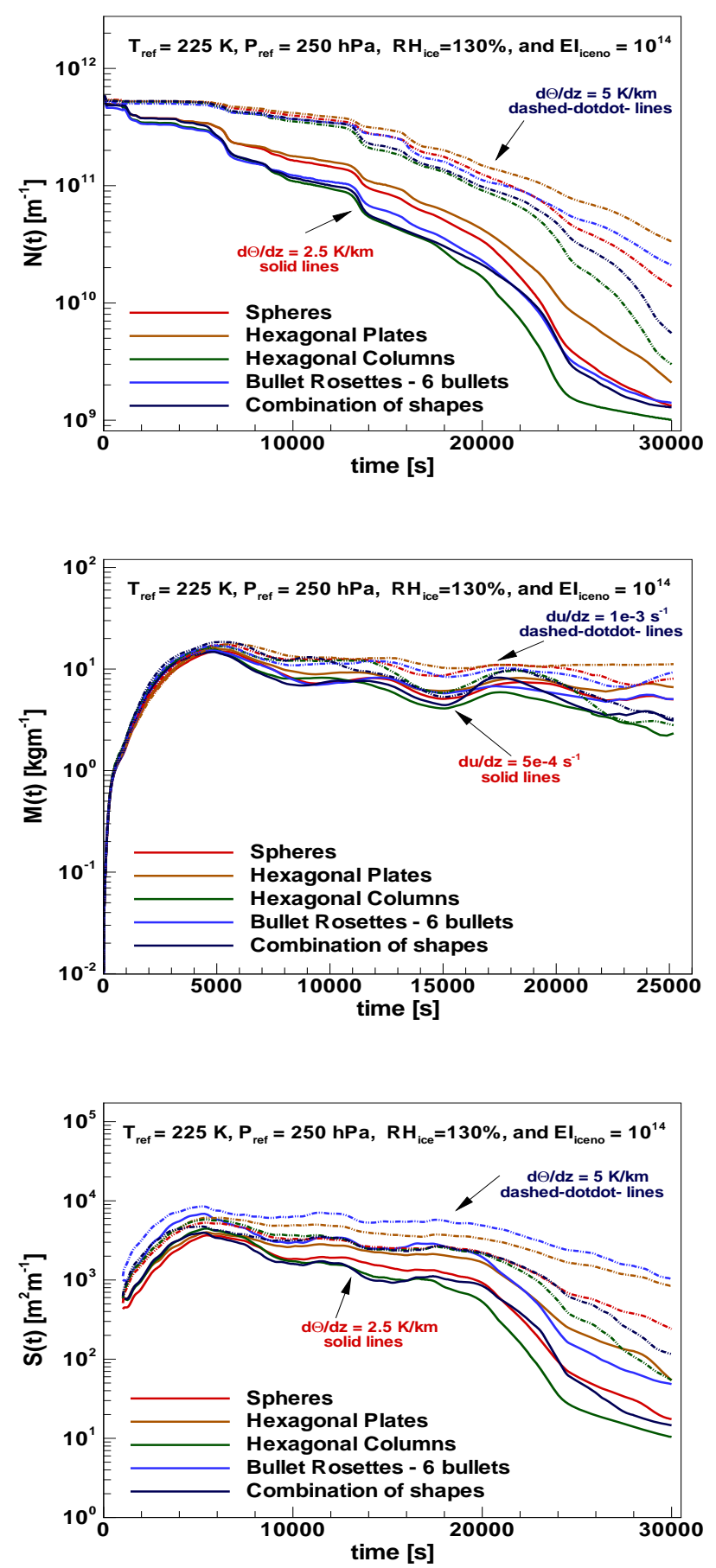

Figure 5.13: Comparison of all shapes. $\mathrm{N}(\mathrm{t}), \mathrm{M}(\mathrm{t})$ and $\mathrm{S}(\mathrm{t})$ - single realizations, Stratification level at late time. Case I. 

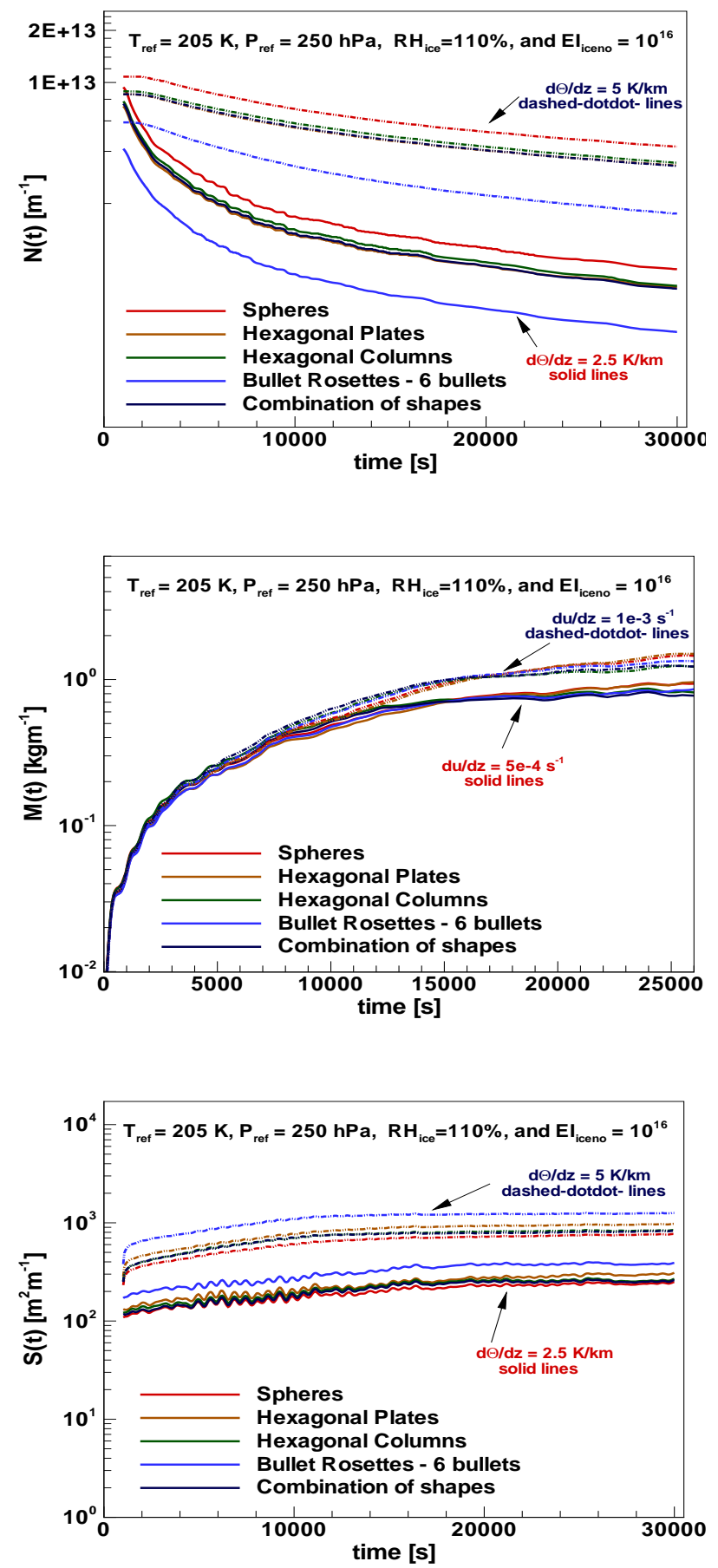

Figure 5.14: Comparison of all shapes. $\mathrm{N}(\mathrm{t}), \mathrm{M}(\mathrm{t})$ and $\mathrm{S}(\mathrm{t})$ - single realizations, Stratification level at late time. Case II. 


\subsection{Shape Effect Analysis and Initial Radiation Study}

Two types of studies on radiation have been performed: Radiative impact of contrail on the atmosphere and impact of radiation on contrail. We are interested in radiation effects on the contrail dynamics and the possible influence of shape effects.

The differences observed in the ice crystal surface area $\mathrm{S}(\mathrm{t})$ in the previous analysis of contrail evolution suggest that it might be more important to consider the shape effect in radiative processes.

The inclusion of radiation on the simulation of older contrails permits a realistic evaluation of contrail evolution. During this process, the ice crystal size distribution changes due to temperature, relative humidity, wind shear, etc. The influence of the ice crystal sizes on radiation processes was tested using the base cases I and II. The radiation process within contrails includes mainly the contribution of infrared radiation rather than solar radiation.

Figures 5.15 and 5.16 present the comparison of all shapes in simulations coupled with radiation performed assuming the surface Earth temperature $T_{s f c}=298 \mathrm{~K}$. The crude approximation of changing only the cross-sectional area with changing crystal shape is the factor important in the results. The results with coupled radiation present a larger spread in the faster falling ice particles (e.g hexagonal columns and combination of shapes) than in the other shapes. On the other hand, in large crystals (base case I), the level of dispersion remains almost constant. The first observation in the base case I (relative large ice particles), is that the difference due to the shape effect is slightly larger compared to the uncertainty level in the ice crystal number $\mathrm{N}(\mathrm{t})$ and ice crystal mass $\mathrm{M}(\mathrm{t})$. The 

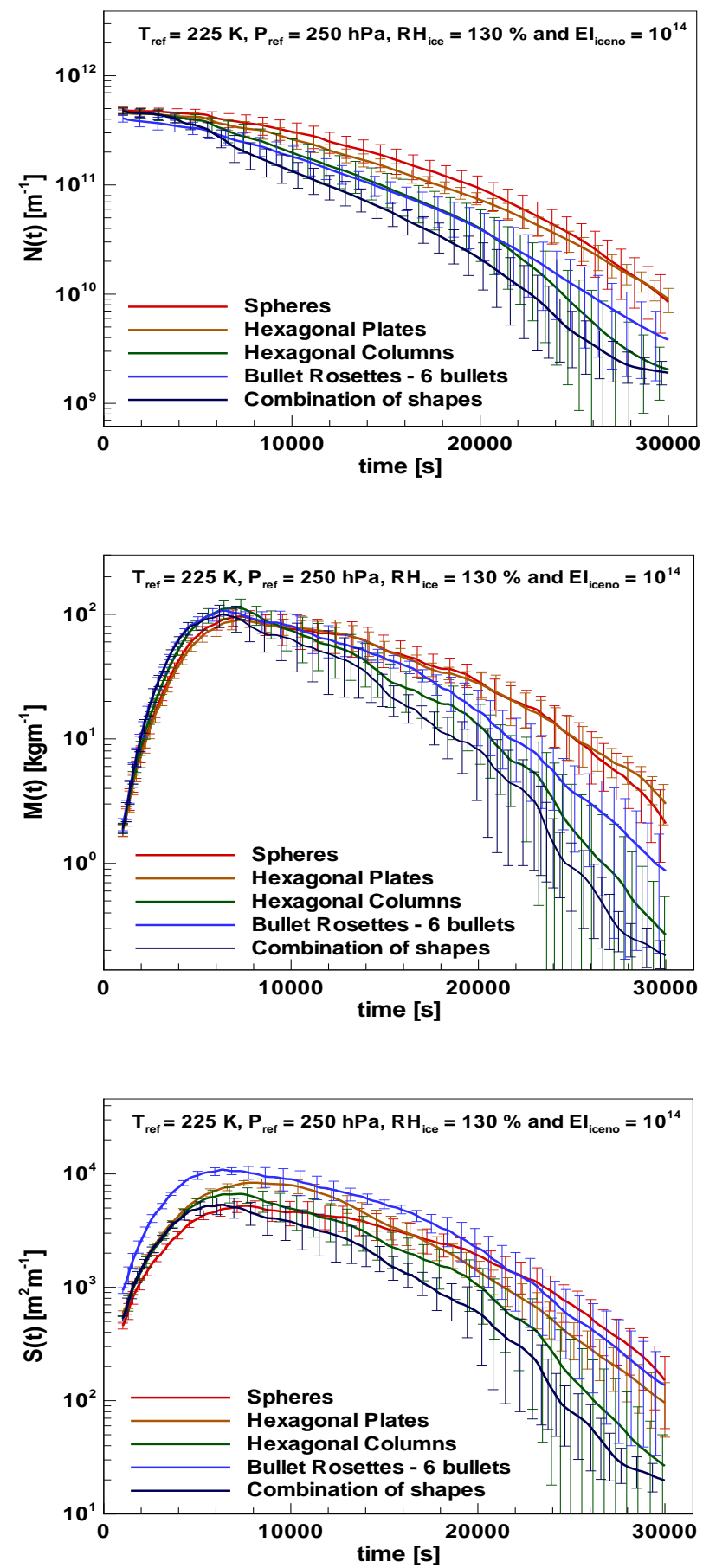

Figure 5.15: Comparison of all shapes. $\mathrm{N}(\mathrm{t}), \mathrm{M}(\mathrm{t})$ and $\mathrm{S}(\mathrm{t})$ - single realizations, Radiation at late time. $T_{s f c}=298 K$. Case I. 

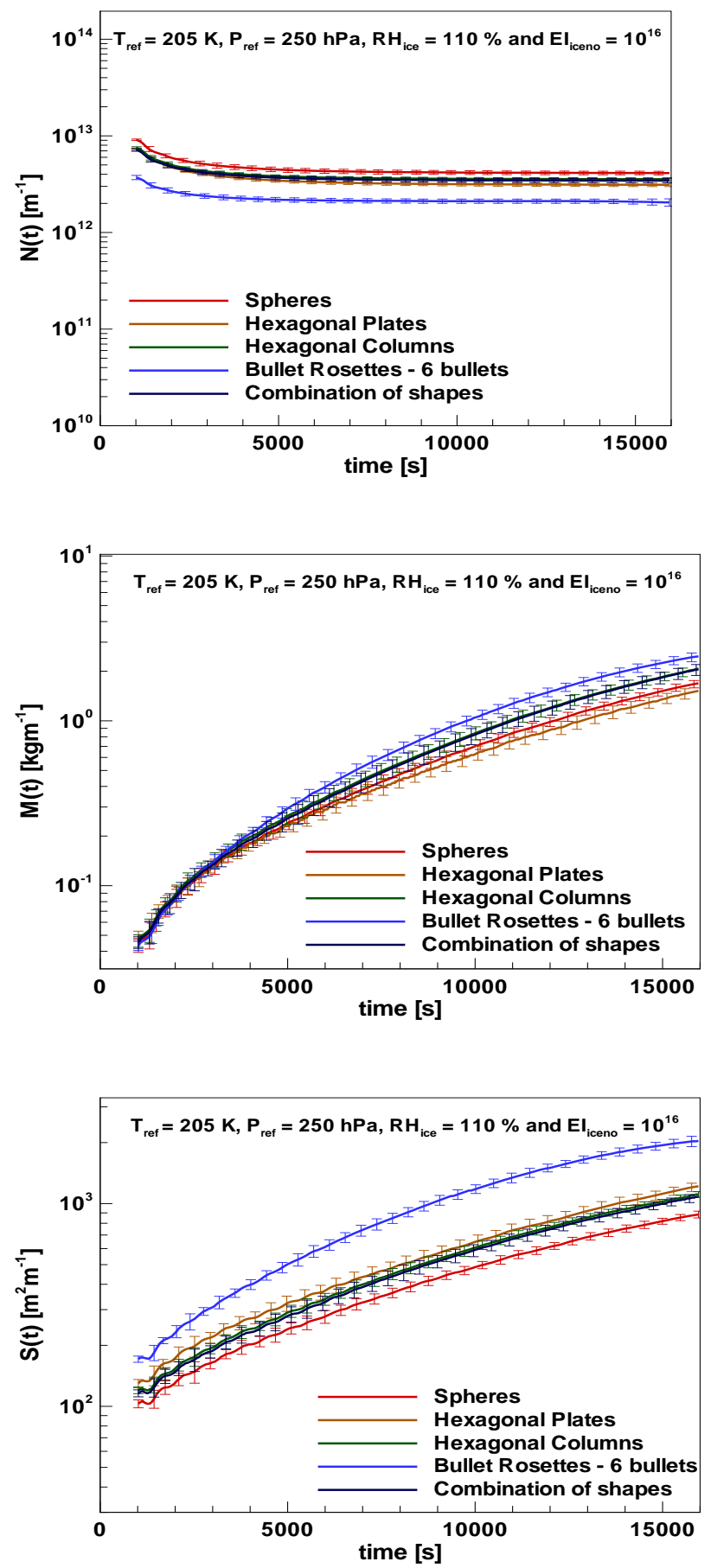

Figure 5.16: Comparison of all shapes. $\mathrm{N}(\mathrm{t}), \mathrm{M}(\mathrm{t})$ and $\mathrm{S}(\mathrm{t})$ - single realizations, Radiation at late time. $T_{s f c}=298 \mathrm{~K}$. Case II. 
significant differences in this specific case are mainly driven by the shape effect (especially in hexagonal columns and combination of shapes). The "precipitation loss" is the dominant mechanism of ice crystals loss during the simulation. The difference in the $\mathrm{S}(\mathrm{t})$ plays an important role in the radiative processes.

In the base case II, the difference by shape effect as in previous cases is larger than the dispersion level. This difference is basically influenced by Kelvin effect "in-situ loss".

\section{Simple Analysis of Contrail Radiation}

Contrail ice particles are characterized to effectively emit/absorb solar and terrestrial radiation (figure 5.17). The shape effect on contrail radiation was treated using the simple analysis developed in (Lewellen et al., 2009) for spherical ice crystals.

The inclusion of the shape effect in the radiative heating by infrared radiation is directly related to the mass absorption coefficient $\kappa^{\uparrow \downarrow}$. In this study a better approximation was used to include the shape effect by Fu et al. (1998) in the infrared spectral range for $D_{e}$ between 11-129.6 $\mu \mathrm{m}$. The parameterization of the mass absorption coefficient was $\kappa^{\uparrow \downarrow}=\beta_{a} / I W C$, where $\beta_{a}$ is the volume absorption coefficient defined as:

$$
\beta_{a}=\frac{I W C}{D_{e}}\left(b_{0}+b_{1} D_{e}+b_{2} D_{e}^{2}+b_{3} D_{e}^{3}\right)
$$

where the constant values of $b_{0}=1.111773, b_{1}=5.7251 e-03, b_{2}=-7.6833 e-$ 05 , and $b_{3}=3.126251 e-07$, were taken at a wavelength of $11 \mu \mathrm{m}$, that frequently represents the entire longwave spectrum. The value of IWC will scale with the equilibrium ice density at flight level, $\rho_{i e}$, which drops strongly with 


\section{Radiative heating/cooling within in contrails}

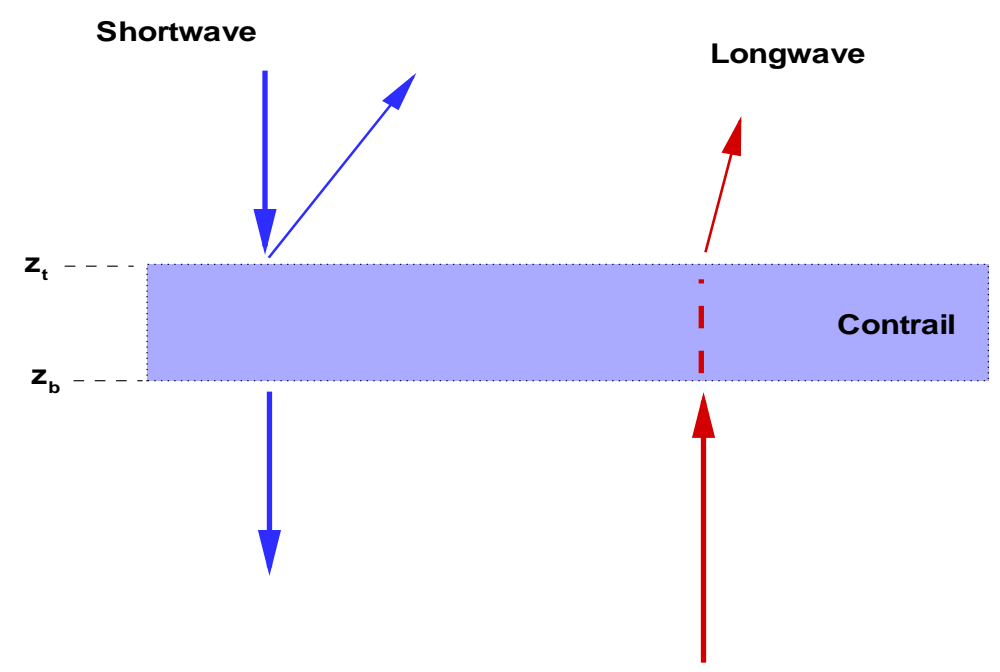

Figure 5.17: Schematic radiation on contrails

contrail temperature $T_{c}$ for fixed $R H_{\text {ice }}$ and $P_{\text {ref }}$.

$$
\rho_{i e}=\left(R H_{i c e} / 100-1\right) q_{s i} \rho_{i}
$$

In this simplified model, the inclusion of the shape effect is through the effective radius $r_{e}$ or effective diameter $D_{e}$, directly related to the cross-sectional area. It is the representation of all spectrum in a clouds. Many studies suggest that the inclusion of IWC-projected area ratio of the size distribution better describes the radiative properties of ice clouds (Mitchell, 2002). This parameter is important since it includes the shape effect in the radiative analysis of contrails. Using the definition of $\mathrm{Fu}$ (1996), and assuming a certain level of ice concentration with 
the same sizes, we obtained the following expression for the effective diameter for non-spherical particles:

$$
D_{e}=2 r_{e}=\frac{2 \sqrt{3} I W C}{3 \rho_{i} A_{c v}}
$$

where $A_{c v}$ is the cross-sectional area per unit volume of a single ice particle.

In our study we will use the effective radius $r_{e}$ in the plots. Figure 5.18 shows the effective radius for all shapes in study covering all our spectrum. Hexagonal columns, bullet rosettes have lower values than spheres, except hexagonal plates, due to the small cross-sectional area in the small ice particles.

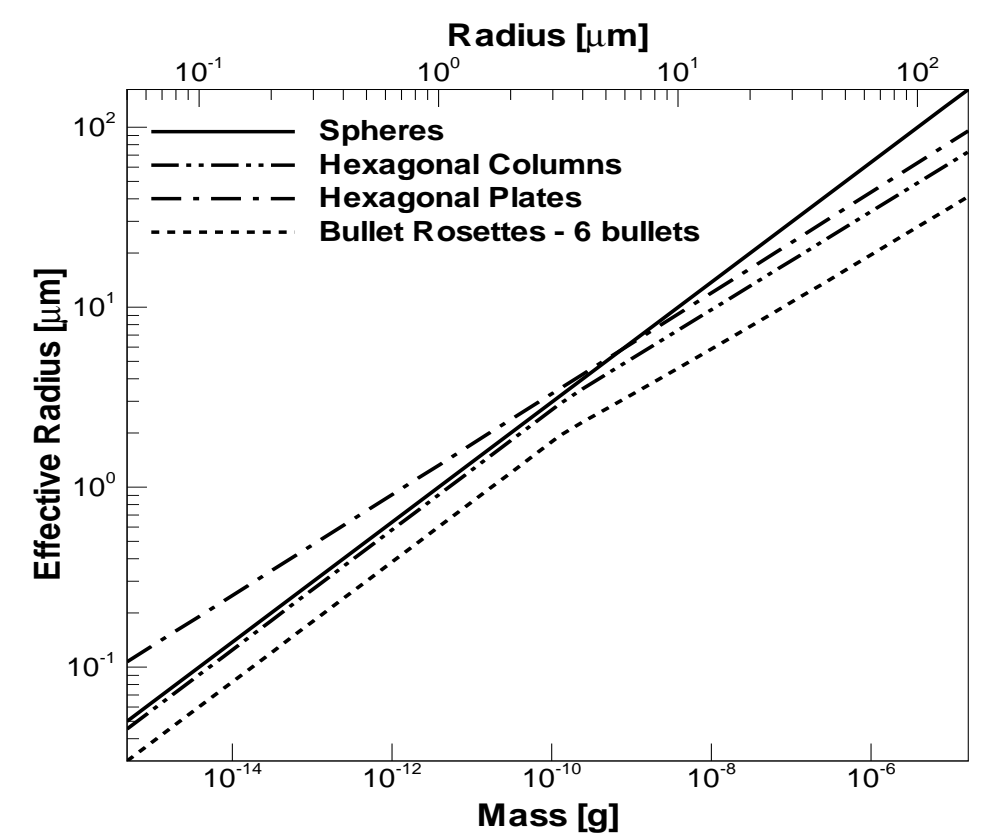

Figure 5.18: Effective radius for the different shapes in study

The simple expression to calculate the volume absorption coefficient is inversely proportional to the effective diameter $D_{e}$. This means that the small ice particles have the highest values of heating rates in agreement with previous studies 
reported by Fu and Liou (1993).

We have calculated the volume absorption coefficient for all our spectrum to analyze the influence of the small and large ice particles. The normalized volume absorption coefficient is shown in figure 5.19. This figure shows the comparison in order of magnitude between all shapes in study. The first observation is that in the range of small ice particles the hexagonal plates have the lowest value of volume absorption coefficient. However, in the part of the spectrum formed by large particles the spheres present the lowest value of volume absorption coefficient.

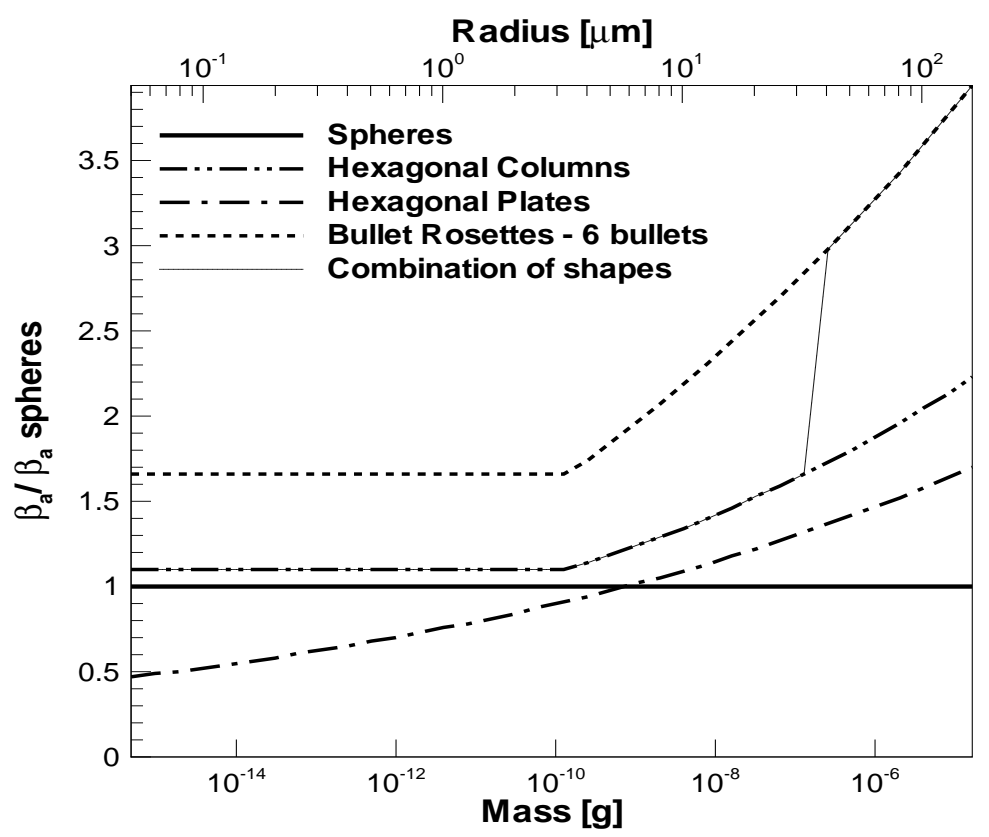

Figure 5.19: Volume Absorption Coefficient normalized by spheres

The effect of the ice crystal habit (shape and size) on the infrared heating rates on contrail simulations (average heating rates): for base case I (relatively large ice particles) and base case II (small ice particles) are illustrated in figures 5.20 and 5.21. The simulations of contrails with coupled radiation were made having 
as starting with spherical results in order to concentrate the analysis on the shape effect in the radiation.

Figures 5.20 and 5.21 show the evolution of the infrared heating rate in time. The figures present two well defined regions: the upper surface of the plume located at flight present a cooling rate by the emission process and in the region of high concentration of ice particles the heating rate. As was expected, the bullet rosette shape presents the highest value of heating rate during all time in the simulations. The simulation of older contrail with small bullet rosette ice crystals is an unphysical case, because of they are still smaller. In both cases, as consequence of heating rate and the warm Earth surface temperature a buoyancy forcing is observed that produces the upward motion of contrail plumes over the flight level. The up shifting process creates with an adiabatic cooling that shifts the saturation level and helps ice particles to grow and increases the contrail volume.

The updraft motion of contrail parcels continues until the precipitation process reaches important levels. This phenomenon in observed in contrails formed with relative large particles. An important observation is that in base case I, the heating rate has large values at the beginning of the simulations $(1 / 2$ hour) when the ice particles are not large enough. It is reduced drastically during the precipitation process. This probably means that the magnitude of the heating rate is directly related to the ice crystal habit (shape and size) and ice concentration. In order to have a more accurate conclusion more investigation is needed.

In the case with small crystals it is observed that the shifting process continues during all simulation since the precipitation process is not present yet. During the simulation it is observed that low temperature and the high moisture level 

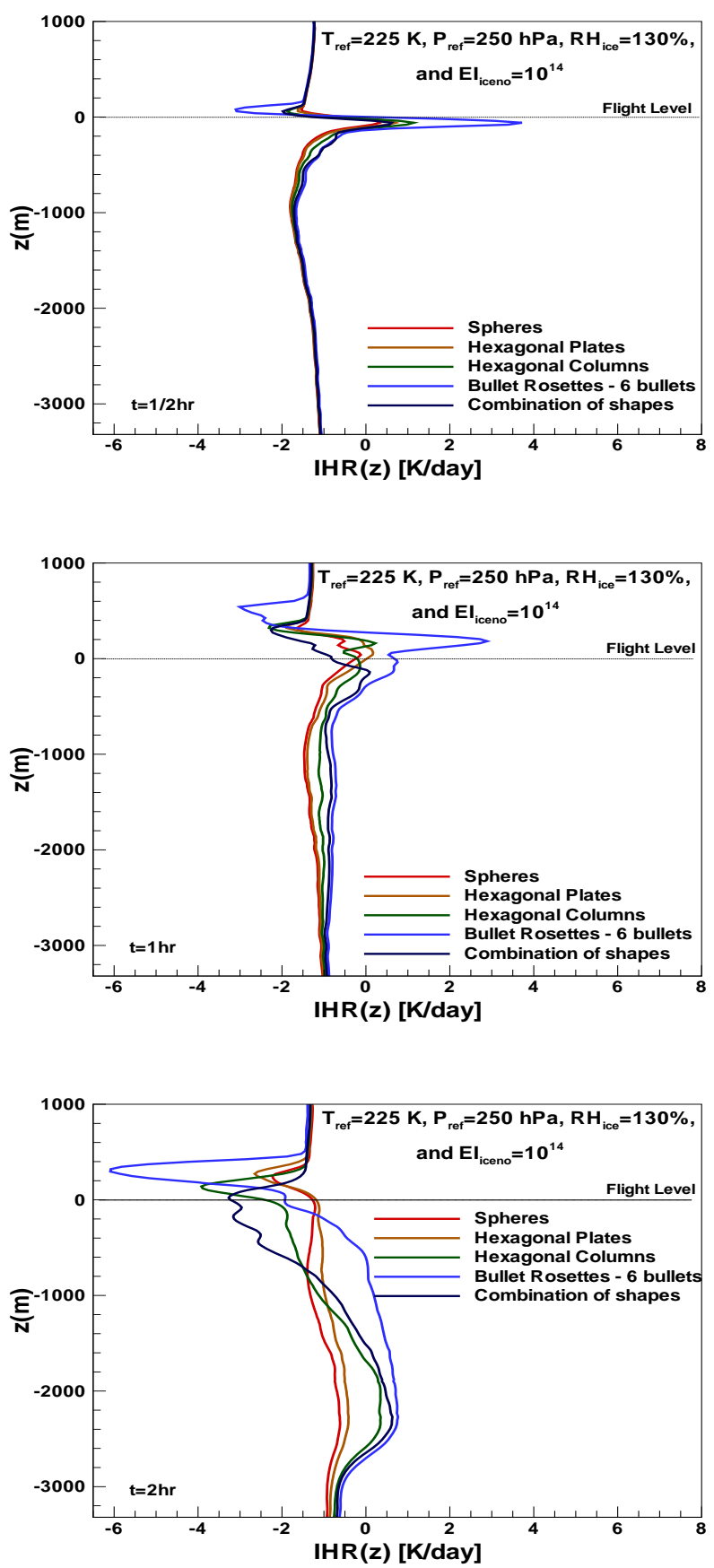

Figure 5.20: Infrared heating rates comparison of all shapes. $\mathrm{N}(\mathrm{t}), \mathrm{M}(\mathrm{t})$ and $\mathrm{S}(\mathrm{t})$ single realizations, Infrared heating rates at late time. $T_{s f c}=298 \mathrm{~K}$. Case I. $\mathrm{t}=1 / 2 \mathrm{hr}$, $2 \mathrm{hr}$ and $3 \mathrm{hr}$ respectively. 

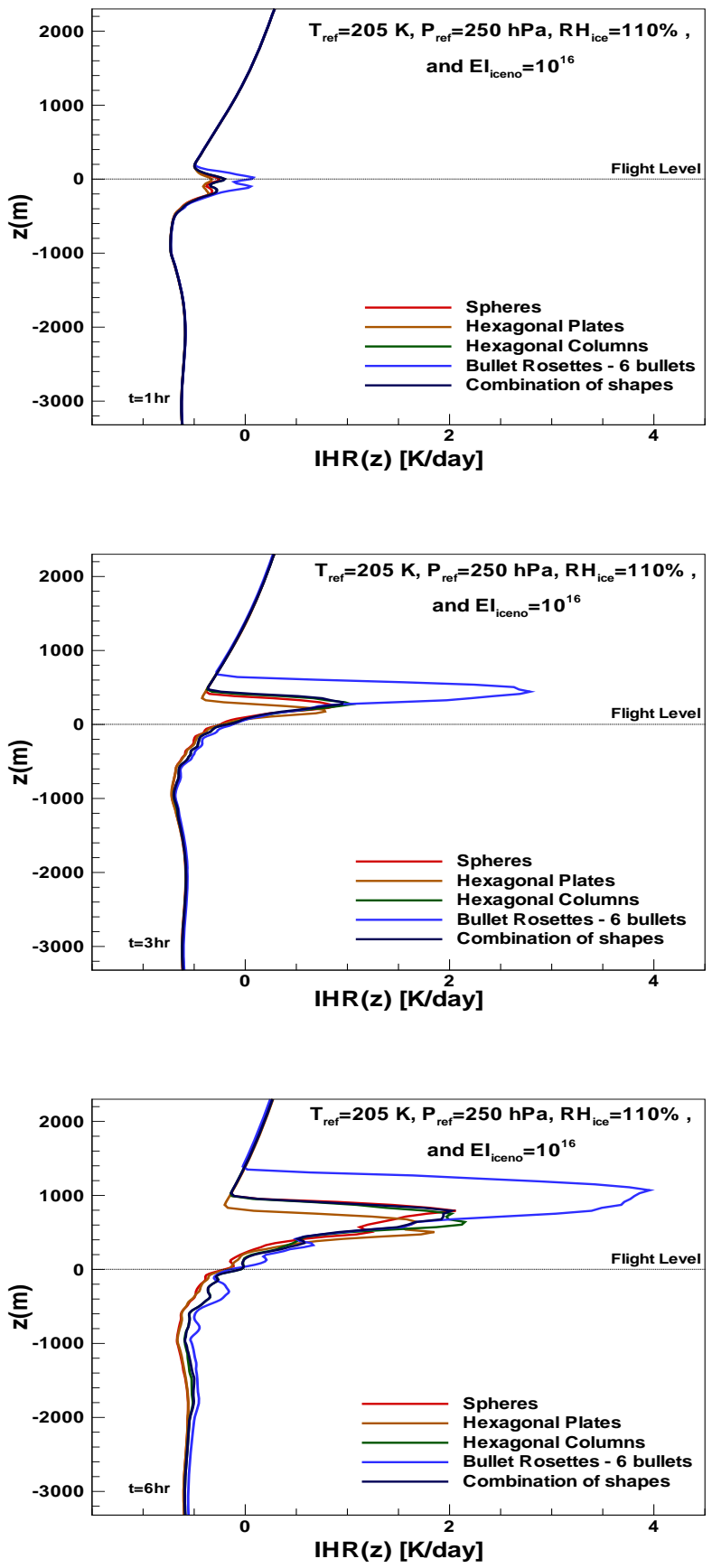

Figure 5.21: Infrared heating rates comparison of all shapes. $\mathrm{N}(\mathrm{t}), \mathrm{M}(\mathrm{t})$ and $\mathrm{S}(\mathrm{t})$ single realizations, Infrared heating rates at late time. $T_{s f_{c}}=298 \mathrm{~K}$. Case II. $\mathrm{t}=1 \mathrm{hr}$, $3 \mathrm{hr}$ and $6 \mathrm{hr}$ respectively. 
(adiabatic cooling) increase the IWC and hence the increase in infrared heating rate. This process was demonstrated in previous studies by Ackerman et al. (1988).

A comparison of the results from the simplified model for the volume absorption coefficient in figure 5.19 (considering a parcel with all ice crystals of the same size) and contrail simulations figures 5.20 and 5.21 can establish that they are in good agreement. In the contrail region of high concentration of ice crystals. Large bullet rosettes ice crystals have higher heating rate followed of hexagonal columns, hexagonal plates and spheres. In small ice crystals, the order is bullet rosettes, hexagonal columns, spheres and hexagonal plates. The case of combination of shapes with large crystals presents a strange behavior that need to be more studied. The shape effect difference in the average heating rates is small on the modest and crude inclusion of the cross-sectional area. The small difference in the number of ice crystals remaining in the plume $\mathrm{N}(\mathrm{t})$, ice surface area $\mathrm{S}(\mathrm{t})$ and infrared heating rate $\operatorname{IHR}(\mathrm{z})$ means that the shape effect could be important in contrail simulations coupled with radiation. A more detailed study of coupled radiation covering a large range of atmospheric parameters allow a final conclusion on the shape effect. A better parameterization is needed for the cross-sectional area since it has an important influence in the radiative calculations. 


\section{Chapter 6}

\section{Initial Sensitivity Analysis of Ice Deposition Coefficient}

\subsection{Introduction}

The shape effect study suggests that the effects of more complex ice crystal shapes in the contrail ice microphysics is not critical and it is limited to simulations coupled with radiation. During the contrail evolution, the number of ice crystals remaining in the plume, $\mathrm{N}(\mathrm{t})$, plays an important role in the lasting effect of persistent contrails. At late time, the evolution of the level of ice concentration is mainly driven by two mechanisms of ice crystal loss: "in-situ" and "precipitation". Previous studies in the formation and evolution of cirrus clouds established that the deposition coefficient $\beta_{i}$ is a critical parameter in the ice crystal number densities produced by homogeneous nucleation (Lin et al., 2002). However, in the evolution of young and older contrails, the "in-situ loss" is the mechanism that involves small ice crystals. This new source of uncertainty was also studied comparing its influence in contrails with large and small ice crystals. 
The initial analysis of ice deposition coefficient $\beta_{i}$ in real contrails was performed for base cases I and II (large and small crystals), as was defined in table 4.3, with spherical ice crystals. The tested values of $\beta_{i}$ were $0.01,0.1,0.3,0.5,0.7$ and 0.93 (default value in CARMA).

\subsection{Young Contrails}

In order to have a clear idea of the level of uncertainty in this parameter, we have performed a study of the level of dispersion with 7 different realizations for each deposition coefficient value $\beta_{i}$. Results from young contrails for the two base cases are shown in figures 6.1 and 6.2 .

The differences in the results by deposition coefficient in $\mathrm{N}(\mathrm{t})$ is large compared with the uncertainty level. The influence of the deposition coefficient is clearly important to contrail evolution.

The results show the expected behavior, where a decrease in the $\beta_{i}$ leads to the conservation of more ice crystals in the plume. The influence of $\beta_{i}$ is more evident in contrails with small ice crystals (figure 6.2). A decrease in $\beta_{i}$ leads to a decrease in the mass growth rate and the "in situ" loss is reduced with more ice crystals surviving in the plume.

\subsection{Older contrails}

Figures 6.3 and 6.4 show the results in older contrails. In these types of contrails, the influence of deposition coefficient decreases in contrails mainly formed by large crystals, since for large crystals the influence of $\beta_{i}$ is minimized. However, in small ice crystals it continues being important. 

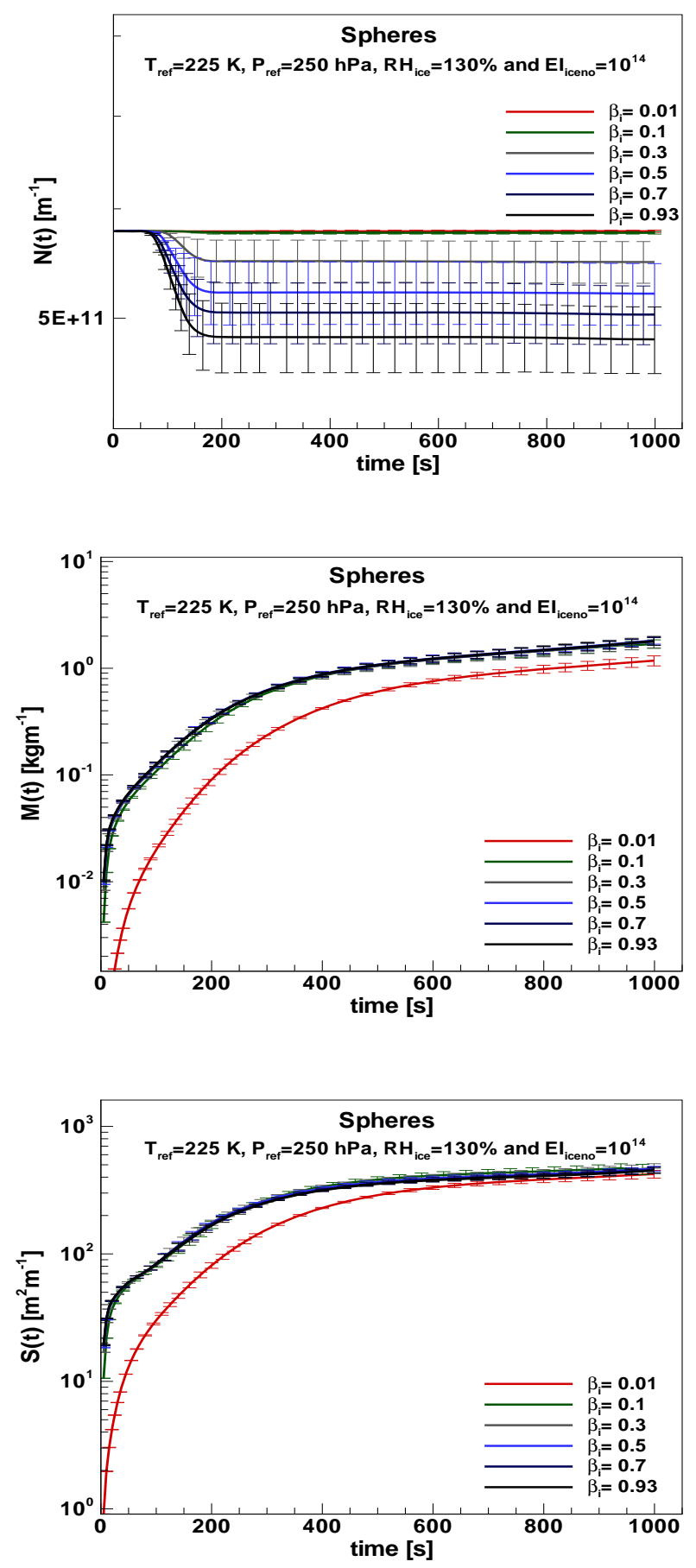

Figure 6.1: Ice Deposition Coefficient $\beta_{i}$, Large Crystals - Analysis - Early time. 0.01 Solid line (average) and bars (uncertainty level) 

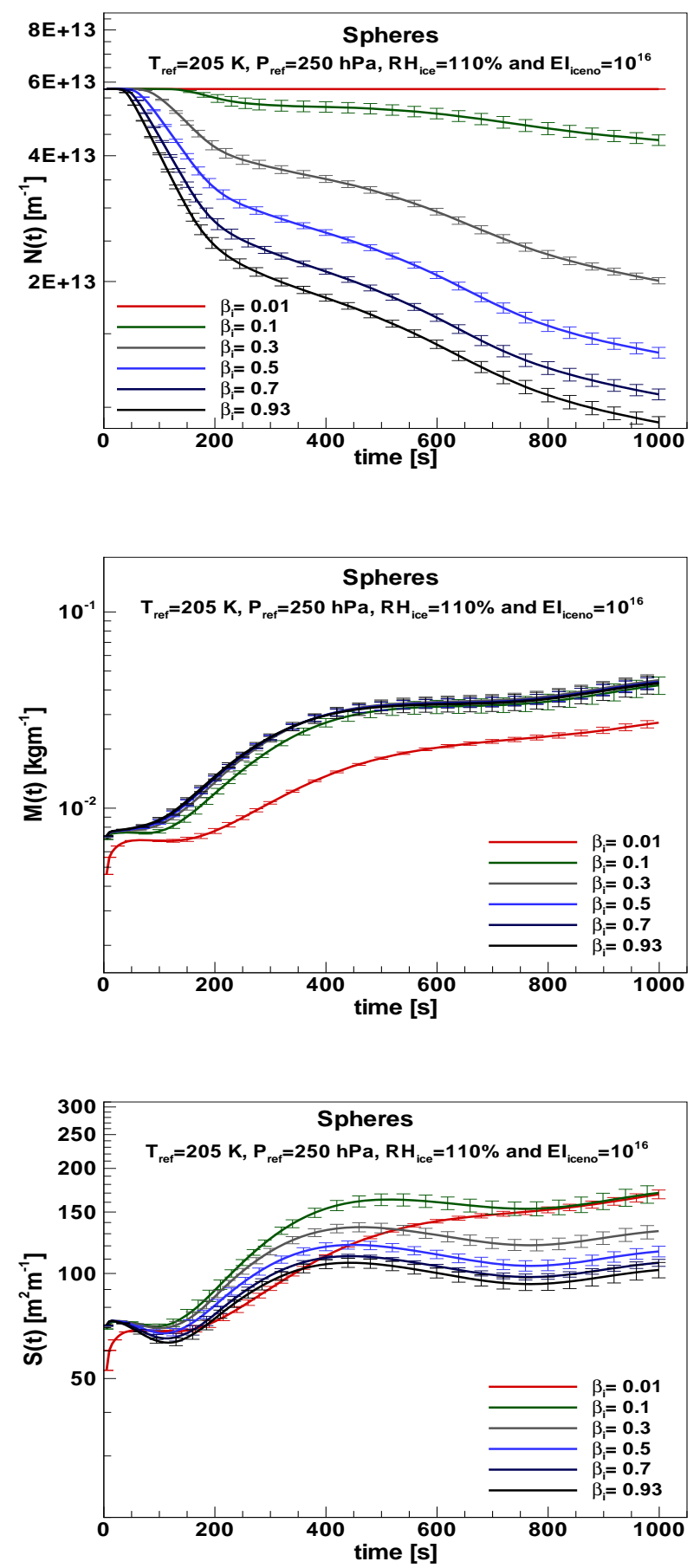

Figure 6.2: Ice Deposition Coefficient $\beta_{i}$, Small Crystals - Analysis - Early time. Solid line (average) and bars (uncertainty level) 
An interesting pattern is observed when the values of $\beta_{i}$ are close to 1 , the ice crystal number $\mathrm{N}(\mathrm{t})$, ice mass $\mathrm{M}(\mathrm{t})$ and ice surface area $\mathrm{S}(\mathrm{t})$ shows little sensitivity in the results. However, an increase in sensitivity is observed at values close to 0.1 in the difference in the number of ice crystals remaining in the plume. Additionally, the results of ice mass $\mathrm{M}(\mathrm{t})$ and ice crystal surface area $\mathrm{S}(\mathrm{t})$ present some wiggles that probably has its origin in the BruntVäisälä oscillation.

(Gierens et al., 2003) in cirrus clouds studies, and (Magee et al., 2006) in laboratory studies suggest $\beta_{i}$ values of less than 0.1. However, Lohmann et al. (2008); Jensen et al. (2005) comparing to other observations and simulations deduce that values $\sim 0.1$ is not appropriate and gives unrealistic results.

Previous studies suggest the dependence of the ice deposition coefficient on crystal size (Gierens et al., 2003), Nelson and Kay (2007), and on ice supersaturation (Wood et al., 2001). It might be interesting to define the limits on $\beta_{i}$ taking account the shape effect. The present results suggest a need for a more detailed study of this parameter, since its potential effect on the number of ice crystals remaining in the contrail plume is significant. 

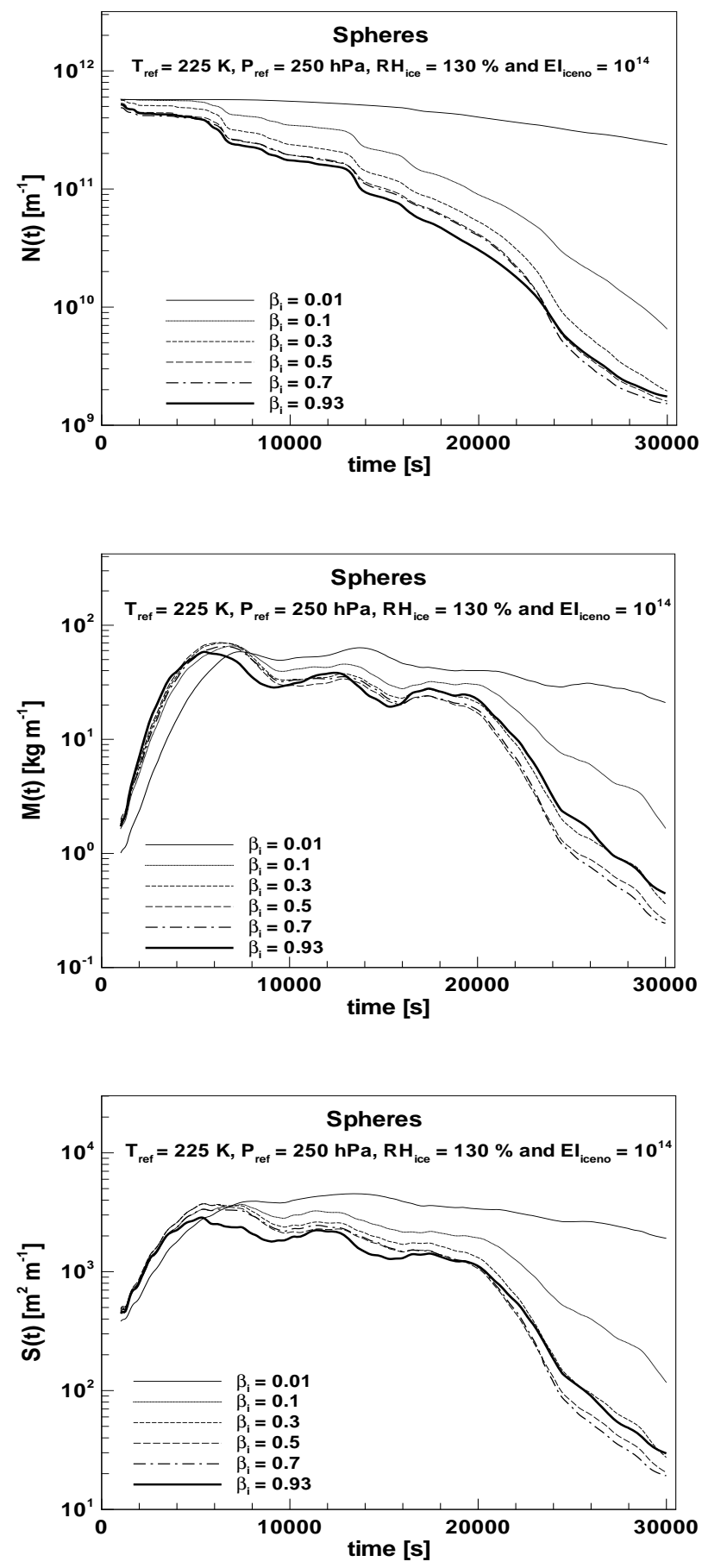

Figure 6.3: Ice Deposition Coefficient $\beta_{i}$, Large Crystals - Analysis - Late time. 0.01 (solid), 0.1 (dotted), 0.3 (short-dash), 0.5 (long-dash), 0.7 (dash-dot), and 0.93 (solidthick) 

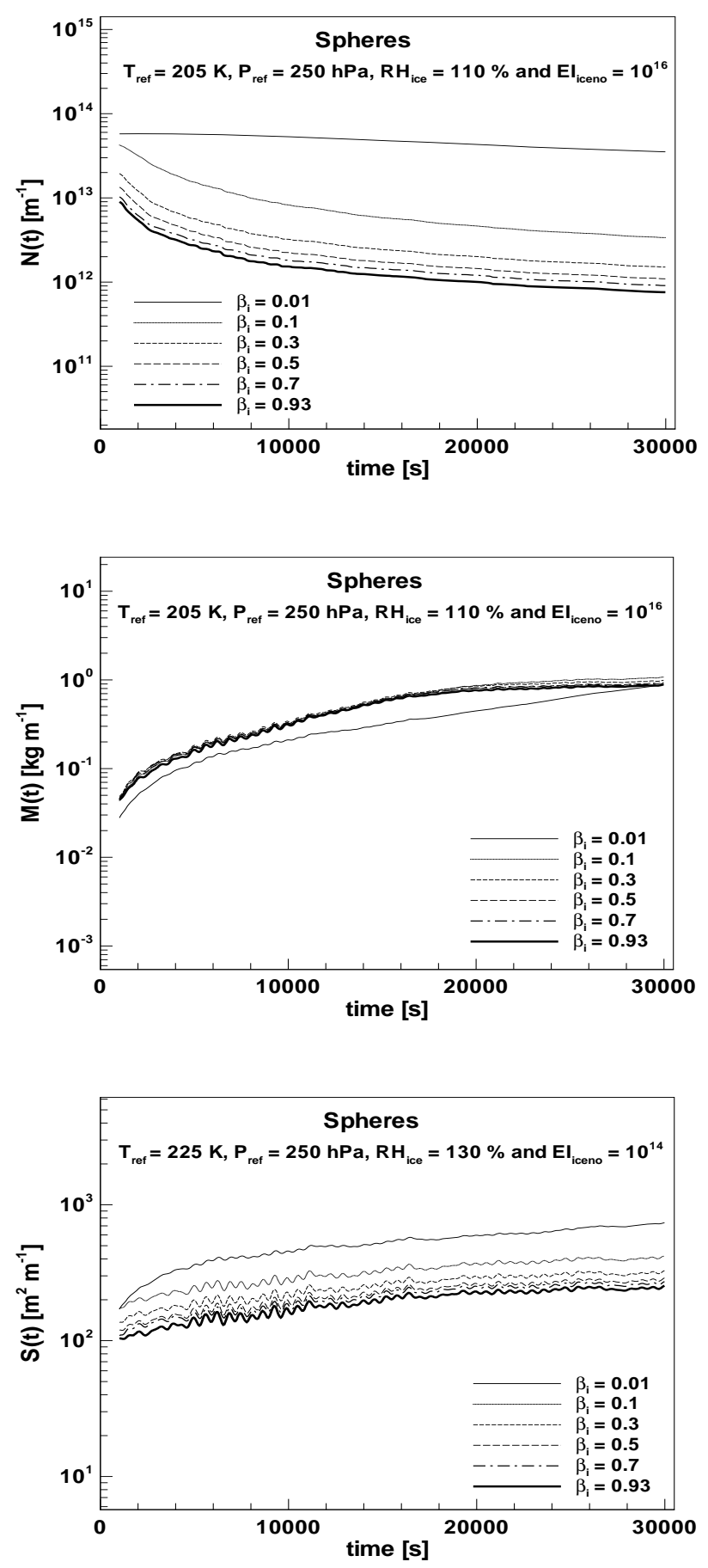

Figure 6.4: Ice Deposition Coefficient $\beta_{i}$, Small Crystals - Analysis - Late time. 0.01 (solid), 0.1 (dotted), 0.3 (short-dash), 0.5 (long-dash), 0.7 (dash-dot), and 0.93 (solidthick) 


\section{Chapter 7}

\section{Conclusions and Future Work}

\subsection{Conclusions}

The primary goal of this study was to determine the sensitivity of contrail behavior to ice crystal shape. This involves the implementation of hexagonal column, hexagonal plate, and bullet rosette ice shapes with the objective to study evolution of contrails in a more realistic manner. In situ measurement and previous studies reported that contrails are formed by ice crystals of different habits, including particles close to spheres, hexagonal columns and bullet rosettes. The potential of an ice spectrum formed by combination of these shapes was implemented and tested in this study. The smallest crystals were small hexagonal columns with aspect ratio 1 (close to spheres), followed by hexagonal columns as particles of middle size (aspect ratio larger than 1) and finally the largest were bullet rosettes.

Results with spheres, hexagonal column, hexagonal plate and bullet rosette crystals and combination of shapes show only modest effects on the contrail evolution without coupled radiation. The maximum difference due to the shape effect in the remaining ice crystal number $\mathrm{N}(\mathrm{t})$ with respect to spheres are $5.3 \%$ 
(hexagonal column), 4.5\% (hexagonal plate), 24.6\% (bullet rosette), and $6.5 \%$ (combination of shapes) for the base case I (large crystals). For small ice crystals (base case II) the maximum differences are 19.4\% (hexagonal column), $22.2 \%$ (hexagonal plate), $59.6 \%$ (bullet rosette), and 21.2\% (combination of shapes). The case with small bullet rosette crystals presents the highest difference in both cases, but this high difference is irrelevant since in situ studies have not reported small well-defined bullet rosette crystals.

In older contrails, the results have a strong dependence on the precipitation level, directly related to the initial configuration of the case under study (initial number of ice crystals, size range spectrum, atmospheric conditions, level of turbulence added, and ice shape). The shape differences are close but with a higher level of uncertainty in the case of large crystals.

Based on the small differences observed in the results we can consider the shape effect in the ice mass $\mathrm{M}(\mathrm{t})$ to be not significant. On the other hand, the ice surface area $\mathrm{S}(\mathrm{t})$ is more sensitive to the shape effect. The differences are basically driven by geometrical rather than dynamical effects.

The differences in $\mathrm{N}(\mathrm{t})$ and $\mathrm{M}(\mathrm{t})$ are not found to be larger for any level of reference temperature $T_{r e f}$, relative humidity with respect to ice $R H_{i c e}$, crossstream wind shear or stratification level considered.

The inclusion of radiation in the contrail simulations increases slightly the difference due to the shape effect compared to the level of dispersion, mainly in the hexagonal and combination of shapes cases. Based on this modest initial study, we can conclude that the shape effect is modest in contrail simulations coupled with radiation. Additional study is needed to analyze the different parametric possibilities coupled with radiation. A better parameterization of the cross-sectional area is needed to reflect the shape effect in a realistic manner. 
The small influence of the shape effect on contrail simulation without coupled radiation suggests that using spherical ice crystals is adequate for modeling contrails at our current level of knowledge. Young and older contrails can be simulated with spherical crystals as the default shape, since the shape effect is negligible. Older contrail with coupled radiation can be initialized from spherical results if necessary.

Based on the initial sensitivity study of $\beta_{i}$ and its influence on the number of ice crystals remaining in the plume, the ice deposition coefficient must be considered as an important source of uncertainty in the current state of the art of modeling of contrail evolution.

\subsection{Future Work}

A realistic treatment of contrails implies a reasonable parameterization of the ice crystals shapes. In order to improve this work the following are the possible tasks that will help to define a better ice shape parameterization:

(a) Model the fall velocity with a better approximation, especially for large ice crystals.

(b) Implement coupled radiation for non-spherical shapes, with the inclusion of cross-sectional area for ice particles of random orientation.

(c) Investigate the influence of atmospheric parameters (cross-stream wind shear and stratification level) on the shape effect difference, in more detail through a parametric study.

(d) Reduce the uncertainty in the value of the deposition coefficient. 


\section{Bibliography}

Ackerman, A. S., O. B. Toon, and P. V. Hobbs, 1995: A model for particle microphysics, turbulent mixing, and radiative transfer in the stratocumulustopped marine boundary layer and comparisons with measurements. J. Atmos. Sci., 52, 1204-1235.

Ackerman, T. P., K. N. Liou, F. P. J. Valero, and L. Pfister, 1988: Heating rates in tropical anvils. J. Atmos. Sci., 45, 1606-1623.

Bailey, M., and J. Hallett, 2004: Growth rates and habits of ice crystals between $-20^{\circ} \mathrm{C}$ and $-70^{\circ} \mathrm{C}$. J. Atmos. Sci., 61, 514-544.

Baumgardner, D., J. E. Dye, B. W. Gandrud, and R. G. Knollenberg, 1992: Interpretation of measurements made by the forward scattering spectrometer probe (fssp-300) during the airborne arctic stratospheric expedition. J. Geophys. Res., 97, 8035-8046.

Baumgardner, D., and B. E. Gandrud, 1998: A comparison of the microphysical and optical properties of particles in an aircraft contrail and mountain wave cloud. Geophys. Res. Lett., 25, 1129-1132.

Boeing, C. A., 2008: Current market outlook 2008-2027. Market Overview.

Chen, J.-P., and R.-F. Lin, 2001: Numerical simulation of contrail microphysi- 
cal and radiative properties. Terrestrial, Atmospheric and Oceanic Sciences (TAO) Journal, 12, 137-154.

Chiruta, M., and P. K. Wang, 2003: The capacitance of rosette ice crystals. J. Atmos. Sci., 60, 836-846.

Chiruta, M., and P. K. Wang, 2005: The capacitance of solid and hollow hexagonal ice columns. Geophys. Res. Lett., 32, L05803.

Chlond, A., 1998: Large-eddy simulation of contrails. J. Atmos. Sci., 55, 796-819.

Crow, S. C., 1970: Stability theory for a pair of trailing vortices. AIAA Journal, 8, 2172-2179.

Ebert, E. E., and J. A. Curry, 1992: A parameterization of ice clouds optical properties for climate models. J. Geophys. Res., 97, 3831-3836.

Fortin, T. J., K. Drdla, L. T. Iraci, and M. A. Tolbert, 2003: Ice condensation on sulfuric acid tetrahydrate: implications for polar stratospheric ice clouds. Journal of Chemical Physics Discussions, 3, 867-894.

Fu, Q., 1996: An accurate parameterization of solar radiative properties of cirrus clouds. JC, 9, 2058-2082.

Fu, Q., and K. N. Liou, 1993: Parameterization of the radiative properties of cirrus clouds. JAS, 50 (13), 2008-2025.

Fu, Q., P. Yang, and W. B. Sun, 1998: An accurate parameterization of the infrared radiative properties of cirrus clouds for climate models. $J C, \mathbf{1 1}$, $2223-2237$. 
Gierens, K., 1996: Numerical simulations of persistent contrails. J. Atmos. Sci., 53, 3333-3348.

Gierens, K., and E. Jensen, 1998: A numerical study of the contrail-to-cirrus transition. Geophys. Res. Lett., 25, 4341-4344.

Gierens, K., M. Monier, and J. F. Gayet, 2003: The deposition coefficient and its role for cirrus clouds. J. Geophys. Res., 108, 4069.

Goodman, J., R. Pueschel, E. Jensen, S. Verma, G. Ferry, S. Howard, S. Kinne, and D. Baumgardner, 1998: Shape and size of contrail particles. Geophys. Res. Lett., 25, 1327-1330.

Hale, B. N., and P. L. M. Plummer, 1974: Molecular model for ice clusters in a supersaturated vapor. Journal of Chemical Physics, 61, 4012-4019.

Hall, W. D., and H. R. Pruppacher, 1976: The survival of ice particles falling from cirrus clouds in subsaturated air. J. Atmos. Sci., 33, 1995-2006.

Haynes, D. R., N. J. Tro, and S. George, 1992: Condensation and evaporation of $\mathrm{H}_{2} \mathrm{O}$ on ice surfaces. J. Phys. Chem., 96, 8502-8509.

Heymsfield, A. J., and J. Iaquinta, 2000a: Cirrus crystal terminal velocities. J. Atmos. Sci., 57, 916-938.

Heymsfield, A. J., and J. Iaquinta, 2000b: Cirrus crystal terminal velocities. $J A S, \mathbf{5 7}, 916-938$.

Heymsfield, A. J., R. P. Lawson, and G. W. Sachse, 1998: Growth of ice crystals in a precipitating contrail. Geophys. Res. Lett., 25(9), 13351338. 
Huebsch, W. W., and D. C. Lewellen, 2006a: Application of LES and binned microphysics for sensitivity study on contrail evolution. Proceedings of the TAC-Conference 167-172.

Huebsch, W. W., and D. C. Lewellen, 2006b: Sensitivity study on contrail evolution. 36th AIAA Fluid Dynamics Conference and Exhibit, 3749, 14.

Jensen, E. J., A. S. Ackerman, D. E. Stevens, O. B. Toon, and P. Minnis, 1998: Spreading and growth of contrails in a sheared environment. J. Geophys. Res., 103 (D24), 31,557-31,567.

Jensen, E. J., L. Pfister, T. Bui, A. Weinheimer, E. Weinstock, J. Smith, J. Pittman, D. Baumgarner, P. Lawson, and M. Mcgill, 2005: Formation of tropopause cirrus layer observed over florida during crystal-face. J. Geophys. Res., 110), D03208.

Ji, W., and P. K. Wang, 1999: Ventilation coefficient for falling ice crystals in the atmosphere at low-intermediate reynolds numbers. Journal of American Meteorological Society, 56, 829-836.

Johnson, D. E., 1997: An examination of microphysical and dynamical processes in a winter storm. PhD thesis, Dept. of Meteorology, University of Winconsin.

Lawson, R. P., A. J. Heymsfield, S. M. Aulenbach, and T. L. Jensen, 1998: Shapes, sizes and light scattering properties of ice crystals in cirrus and persistent contrails during success. Geophys. Res. Lett., 25(9), 1331-1334.

Lewellen, D. C., W. W. Huebsch, O. E. Meza, S. L. Baughcum, and M. Y. Danilin, 2009: Large-eddy simulations of contrails and contrail cirrus with binned microphysics from 1s to several hours In preparation. 
Lewellen, D. C., W. Lewellen, and J. Xia, 2000: The influence of a local swirl ratio on tornado intensification near the surface. J. Atmos. Sci., 57, 527-544.

Lewellen, D. C., and W. S. Lewellen, 1996: Large-eddy simulations of the vortex-pair breakup in aircraft wakes. Journal of American Institute of Aeronautics and Astronautics, 34(11), 2337-2345.

Lewellen, D. C., and W. S. Lewellen, 1998: Large-eddy boundary layer entrainment. J. Atmos. Sci., 55, 2645-2665.

Lewellen, D. C., and W. S. Lewellen, 2001a: The effects of aircraft wake dynamics on contrail development. J. Atmos. Sci., 58, 390-406.

Lewellen, D. C., and W. S. Lewellen, 2001b: The effects of aircraft wake dynamics on measured and simulated $N O_{x}$ and $H O_{x}$ wake chemistry. J. Geophys. Res., 106, 27661-27672.

Lewellen, D. C., and W. S. Lewellen, 2002: Entrainment and decoupling relations for cloudy boundary layers. J. Atmos. Sci., 59, 2966-2986.

Lewellen, D. C., W. S. Lewellen, L. R. Poole, T. J. DeCoursey, G. M. Hansen, and C. A. Hostetler, 1998: Large-eddy simulations and lidar measurements of the vortex pair breakup in aircraft wakes. AIAA Journal, 36, 1439-1445.

Lewellen, W. S., D. C. Lewellen, and R. Sykes, 1997: Large-eddy simulation of a tornado's interaction with the surface. J. Atmos. Sci., 54, 581-605.

Libbrecht, K. G., 2005: The physics of snow crystals. Report on Progress in Physics, 68, 855-895.

Lin, R. F., D. O. Starr, P. DeMott, R. Cotton, K. Sassen, E. Jensen, B. Kärcher, and X. Liu, 2002: Cirrus parcel model comparison project. phase 1: The 
critical components to simulate cirrus initiation explicitly. J. Atmos. Sci., 59.

Liou, K. N., P. Yang, Y. Tahano, K. Sassen, T. Charlock, and W. Arnott, 1998: On the radiative properties of contrail cirrus. Geophys. Res. Lett., 25, $1161-1164$.

Liu, H., P. Wang, and R. Schlesinger, 2003: A numerical study of cirrus clouds. part ii: Effects on ambient temperature, stability, radiation, ice microphysics, and microdynamics on cirrus evolution. J. Atmos. Sci., 60(9), 1097-1119.

Lohmann, U., P. Spichtinger, J. E., P. T., and H. Smit, 3(2008): Cirrus cloud formation and ice supersaturated regions in a global climate model. Environ. Res. Lett., 045022.

MacDonald, J. E., 1963: Use of the electrostatic analogy in studies of ice crystal growth. ZAMP, 14, 610-620.

Magee, N. B., M. A. M., and D. Lamb, 2006: Effect of supersonic aircraft on cirrus formation and climate. 12th Conference on Cloud Physics, Madison, WI.

Magono, C., and C. W. Lee, 1966: Meteorological classification of natural snow crystals. J. Fac. Sci, 7(2), 320-335.

Meerkötter, R., U. Schumann, D. R. Doelling, P. Minnis, T. Nakajima, and Y. Tsushima, 1999: Radiative forcing by contrails. Annales-Geophysicae, 17, 1080-1094.

Meyer, R., H. Mannstein, R. Meerktter, U. Schumann, and P. Wendling, 2002: Regional radiative forcing by line-shaped contrails derived from satellite data. J. Geophys. Res., 107 (17), 1-15. 
Mitchell, D. L., 2002: Effective diameter in radiation transfer: General definition, applications and limitations. J. Atmos. Sci., 59, 2330-2346.

Nelson, J. T., and J. E. Kay, 2007: Towards a realistic deposition coefficient expression for cirrus cloud modeling. American Geophysical Union, Fall Meeting, Madison, WI.

Parungo, F., 1995: Ice crystals in high clouds and contrails. Journal of Atmospheric Research, 38, 249-262.

Penner, J. E., D. H. Lister, D. J. Griggs, D. J. Dokken, and M. McFarland, 1999: . Aviation and the Global Atmosphere: Special Report of the Intergovernmental Panel on Climate Change, IPCC, Cambridge University Press, Cambridge.

Podzimek, J., 1996: Experimental determination of the capacity of ice crystals. Studia Geophys. Geodet., 10, 235-238.

Poellot, M. R., W. P. Arnott, and J. Hallett, 1999: In-situ observations of contrail microphysics and implications for their radiative impact. J. Geophys. Res., 104, 12,077 12,084.

Pruppacher, H. R., and J. D. Klett, 1997: Microphysics of Clouds and Precipitation. Kluwer Academic Publishers, 954 pp.

Schröder, F., B. Kärcher, C. Duroure, J. Ström, A. Petzold, J.-F. Gayet, B. Strauss, P. Wendling, and S. Borrmann, 2000: On the transition of contrails into cirrus clouds. J. Atmos. Sci., 57, 3097-3103.

Schumann, U., and P. Wendling, 1990: Air traffic and the environment background, tendencies and potential global atmospheric effects. Springer-Verlag 245. 
Schummann, U., 1996: On conditions from contrails formation from aircraft exhaust. Meteor. Z. N. F., 5, 3-22.

Spichtinger, P., and K. M. Gierens, 2008: Modelling of cirrus clouds - part 1: Model description and validation. Atmospheric Chemistry and Physics Discussions, 8, 601-686.

Spinhirne, J. D., W. Hart, and D. P. Duda, 1998: Evolution of morphology and microphysics of contrail cirrus from airborne remote sensing. Geophys. Res. Lett., 25, 1153-1156.

Sussmann, R., and K. M. Gierens, 1999: Lidar and numerical studies on the different evolution of vortex pair and secondary wake in young contrails. J. Geophys. Res., 104(D2), 2131-2142.

Sykes, R., and D. S. Henn, 1989: Large eddy simulation of turbulent sheared convection. J. Atmos. Sci., 46(8), 1106-1118.

Sykes, R. I., L. W. S., and D. S. Henn, 1990: Numerical simulation of the boundary layer eddy structure during the cold-air outbreak of GALE IOP-2. Monthly Weather Review, 118(2), 363-374.

Toon, O., T. R. P., J. J., G. J., and G. Ferry, 1989: Physical processes in polar stratospheric ice clouds. J. Geophys. Res., 94, 11359-11380.

Travis, D. J., A. M. Carleton, and R. G. Lauritsen, 2004: Regional variations in U.S. diurnal temperature range for the 11-14 september 2001 aircraft groundings: Evidence of jet contrail influence on climate. Journal of Climate, 17, $1123-1134$.

Unterstrasser, S., K. M. Gierens, and P. Spichtinger, 2008: The evolution of 
contrail microphysics in the vortex phase. Metereologische Zeitschrift, 17(2), $145-156$.

Westbrook, C. D., 2007: Improved estimates for the fall speed of sub-100 $\mu \mathrm{m}$ ice crystals. Quartely Journal of the Royal Meteorological Society, 00, 1-9.

Westbrook, C. D., H. R. J., and A. J. Illingworth, 2008: The capacitance of pristine ice crystals and aggregate snowflakes. J. Atmos. Sci., 65, 206-219.

Wood, S. E., M. B. Baker, and D. Calhoun, 2001: New model for the vapor growth of hexagonal ice crystals in the atmosphere. J. Geophys. Res., 106, $4845-4870$.

Yang, P., G. Hong, A. E. Dessler, S. Ou, K.-N. Liou, P. Minnis, and Hashvardhan, 2008: Contrail and induced cirrus: Optics and radiation. Bull. Am. Meteorol. Soc. 